\title{
HYDROLOGIC DATA \\ FOR THE JEMEZ MOUNTAINS, NEW MEXICO
}

By Cynthia G. Abeyta and Bruce M. Delaney

U.S. GEOLOGICAL SURVEY

Open-File Report 90-176

Prepared in cooperation with the

U.S. BUREAU OF INDIAN AFFAIRS and the NEW MEXICO STATE ENGINEER OFFICE

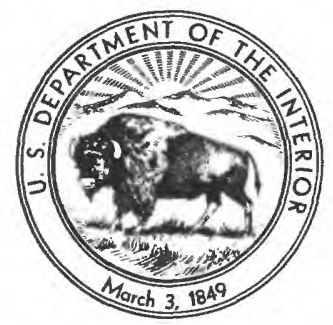




\author{
U.S. DEPARTMENT OF THE INTERIOR \\ MANUEL LUJAN, JR., Secretary \\ U.S. GEOLOGICAL SURVEY \\ Dallas L. Peck, Director
}

For additional information write to:

District Chief

U.S. Geological Survey

Pinetree Office Park

4501 Indian School Road NE, Suite 200

Albuquerque, New Mexico 87110
Copies of this report can be purchased from:

U.S. Geological Survey Books and Open-File Reports Federal Center, Building 810 Box 25425

Denver, Colorado 80225 
Abstract $\ldots \ldots \ldots \ldots \ldots \ldots \ldots \ldots \ldots \ldots \ldots \ldots \ldots \ldots \ldots \ldots \ldots \ldots \ldots \ldots \ldots \ldots \ldots \ldots \ldots$

Introduction $\ldots \ldots \ldots \ldots \ldots \ldots \ldots \ldots \ldots \ldots \ldots \ldots \ldots \ldots \ldots \ldots \ldots \ldots \ldots \ldots$

Numbering system for surface- and ground-water sites .......... 4

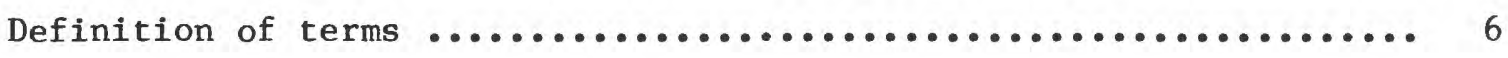

Explanation of hydrologic-data tables $\ldots \ldots \ldots \ldots \ldots \ldots \ldots \ldots \ldots \ldots \ldots \ldots \ldots$

References cited $\ldots \ldots \ldots \ldots \ldots \ldots \ldots \ldots \ldots \ldots \ldots \ldots \ldots \ldots \ldots \ldots \ldots \ldots \ldots \ldots \ldots \ldots$

\section{PLATES}

[In pocket]

Plate 1. Map of the Jemez Mountains study area showing location of surface-water data-collection sites

2. Map of the Jemez Mountains study area showing location of ground-water data-collection sites

\section{FIGURES}

Figure 1. Map showing the location of the Rio Grande rift and the Jemez lineament in New Mexico .......................

2. Map showing the location of the Jemez Mountains study

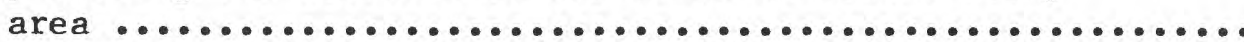

3. Diagram showing sectionized land system of numbering wells and springs in New Mexico

\section{TABLES}

Table 1. Summary of surface-water data-collection sites in the Jemez Mountains, New Mexico ...................... 10

2. Summary of ground-water data-collection sites in the Jemez Mountains, New Mexico ....................... 31 


\section{CONVERSION FACTORS AND VERTICAL DATUM}

Multiply

inch

foot

mile

acre

square mile

gallon

cubic foot

acre-foot

cubic foot per second

gallon per second

gallon per minute

ton per day
By

25.4

0.3048

1.609

0.004047

2.590

3.785

0.02832

0.001233

0.02832

3.785

0.06309

0.9072
To obtain

millimeter

meter

kilometer

square kilometer

square kilometer

liter

cubic meter

cubic hectometer

cubic meter per second

liter per second

liter per second

megagram per day

Temperature in degrees Celsius $\left({ }^{\circ} \mathrm{C}\right)$ can be converted to degrees Fahrenheit $\left({ }^{\circ} \mathrm{F}\right)$ as follows:

$$
{ }^{\circ} \mathrm{F}=9 / 5\left({ }^{\circ} \mathrm{C}\right)+32
$$

Sea level: In this report "sea level" refers to the National Geodetic Vertical Datum of 1929 (NGVD of 1929)--a geodetic datum derived from a general adjustment of the first-order level nets of the United States and Canada, formerly called Sea Level Datum of 1929. 


\title{
HYDROLOGIC DATA FOR THE JEMEZ MOUNTAINS, NEW MEXICO
}

\author{
By Cynthia G. Abeyta and Bruce M. Delaney
}

\begin{abstract}
The Jemez Mountains volcanic complex in north-central New Mexico is an area of geothermal-energy exploration. This report is part of a study by the U.S. Geological Survey of the Jemez geothermal hydrologic system. Hydrologic data for the area were retrieved from the Survey's National Water-Data Storage and Retrieval (WATSTORE) System. Summaries of the availability of peak-flow, water-quality, and daily-values data for 100 surface-water sites, and well data and summaries of the availability of water-quality and water-level data for 294 ground-water sites are presented.
\end{abstract}

\section{INTRODUCTION}

The Jemez Mountains, a large dormant volcanic complex, are in northcentral New Mexico at the intersection of the north-trending Rio Grande rift and the northeast-trending Jemez lineament (fig. 1). The region is a location for geothermal activity, as indicated by several bubbling pools of muddy acid sulfate water, fumaroles, and the many hot springs in the area.

The Jemez Mountains cover an area of about 1,900 square miles (fig. 2) and have a relief of about 6,100 feet. The main streams approximately bounding the Jemez Mountains are the Rio Chama to the north, the Rio Puerco to the northwest, a second Rio Puerco to the west, the Jemez River to the west and south, and the Rio Grande to the east.

This report was prepared as part of a study of the Jemez geothermal hydrologic system conducted by the U.S. Geological Survey in cooperation with the U.S. Bureau of Indian Affairs and the New Mexico State Engineer Office. The report contains a compilation of data or description of data available from the Geological Survey's National Water-Data Storage and Retrieval (WATSTORE) System. The data, which include those collected by the Geological Survey during the Jemez geothermal study, are from the beginning of record through water year 1983. Data in this report indicated as available may be obtained by contacting the New Mexico District Office, U.S. Geological Survey, Water Resources Division. Additional sources of data are cited in an annotated bibliography by Abeyta and Delaney (1986, p. 130). 


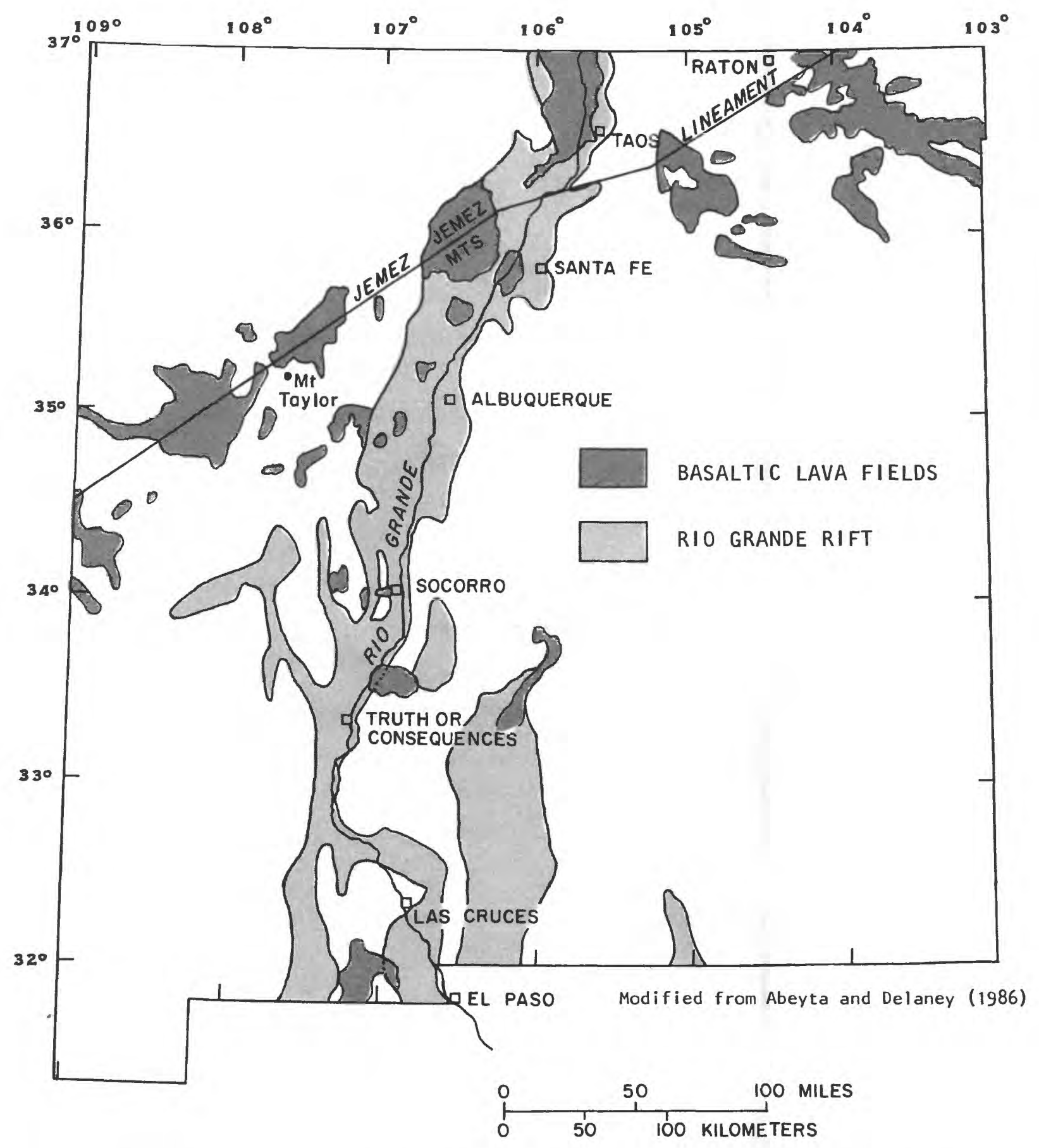

Figure 1.--Location of the Rio Grande rift and the Jemez 1 ineament in New Mexico. 


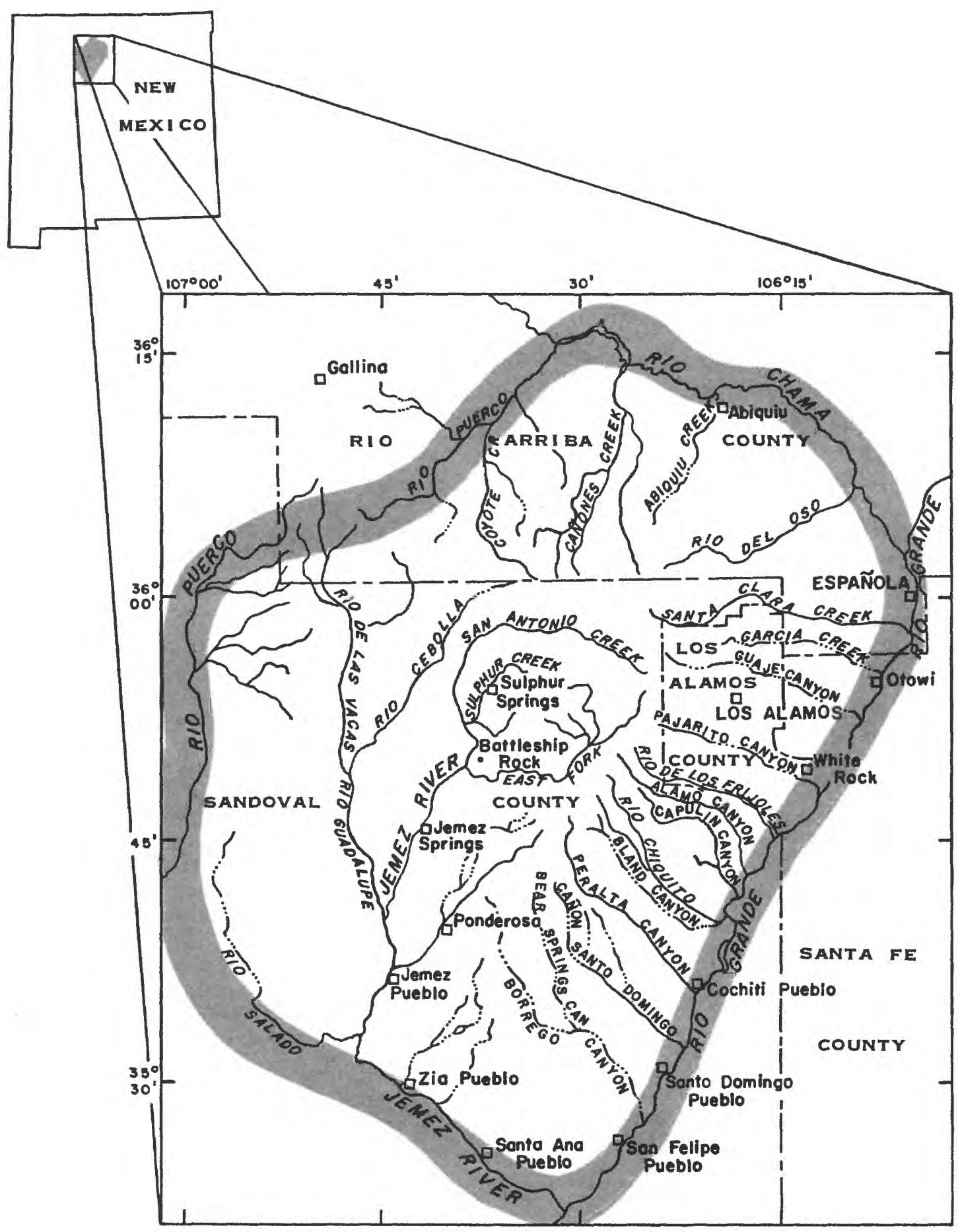

Modified from Abeyta and Delaney, 1986

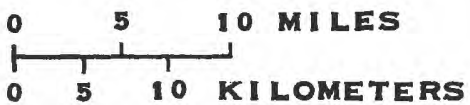

STUDY AREA BOUNDARY

Figure 2.--Location of the Jemez Mountains study area. 
Numbering System for Surface- and Ground-Water Sites

Each surface- and ground-water site in this report is assigned two unique numbers. The first number, as shown in tables 1 and 2, is the map number assigned by the authors. This number is plotted on plates 1 or 2 in order to locate the site. Map numbers for the 100 surface-water sites range from 1 through 100. Map numbers for the 294 ground-water sites range from 101 through 394.

The second unique number is the U.S. Geological Survey station number assigned by Survey personnel. Surface-water sites are assigned either an 8digit number such as 08292000 or a 15-digit number such as 361212106202110 . The eight-digit number represents either a continuous-record station or a partial-record station. No distinction is made between the two. The first two digits, 08, in the eight-digit number represent part 08, the Western Gulf of Mexico Basin. All surface-water records in this report are in part 08. The last six digits in the station number represent a downstream-order number.

Miscellaneous surface- and ground-water stations (including both wells and springs) are given a 15-digit station number. The 15-digit number is based on the grid system of latitude and longitude: the first six digits represent the degrees of latitude, the next seven digits represent the longitude, and the last two digits sequentially identify sites within a 1 -second grid.

To further identify the surface- and ground-water sites, a station identifier under the heading "Station name" or "Local identifier" also is given. The name of a stream, well, spring, or other identifiable source may be included in the station name or local identifier (such as Arroyo Seco near Abiquiu, N. Mex., or Cañon de San Diego Grant Crow Spring). Another means of identification is the public-land survey method of township, range, and section location (fig. 3). An example is $15 \mathrm{~N} .01 \mathrm{~W} \cdot 10.1411$, where $15 \mathrm{~N}$. denotes Township 15 North, 01W. denotes Range 1 West, 10 denotes section 10, and 1411 denotes the tract, northwest $\frac{1}{4}$ of the northwest $\frac{1}{4}$ of the southeast $\frac{1}{4}$ of the northwest $\frac{1}{4}$ of section 10 . 


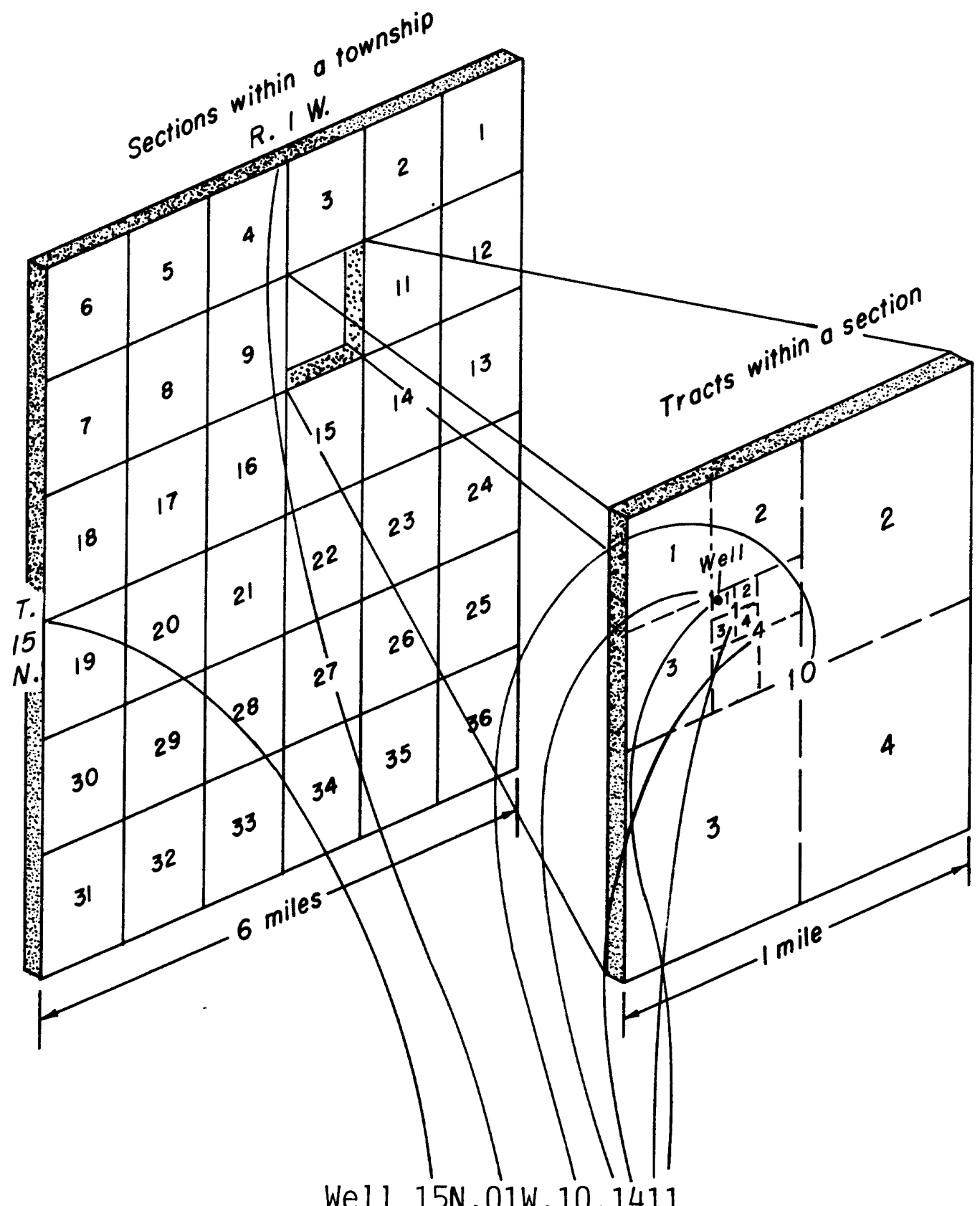

Well 15N.01W.10.1411

Figure 3.--Sectionized land system of numbering wells and springs in New Mexico. 


\section{Definition of Terms}

Terms related to streamflow, water-quality, and other hydrologic data in this report are defined below (U.S. Geological Survey, 1984).

Acre-foot: The quantity of water required to cover 1 acre to a depth of 1 foot, equivalent to 43,560 cubic feet or 325,851 gallons or $1,233.49$ cubic meters.

Aquifer: A geologic formation, group of formations, or part of a formation that contains sufficient saturated permeable material to yield significant quantities of water to wells and springs.

Cubic foot per second: The rate of discharge representing a volume of 1 cubic foot passing a given point during 1 second, equivalent to approximately 7.48 gallons per second or 448.8 gallons per minute or 0.02832 cubic meter per second.

Discharge: The volume of water (or more broadly, volume of fluid plus suspended sediment) that passes a given point within a given period of time.

Mean discharge: The arithmetic mean of individual daily mean discharges during a specific period.

Instantaneous discharge: The discharge at a particular instant of time.

Drainage area: Of a stream at a specific location is that area, measured in a horizontal plane, enclosed by a topographic divide from which direct surface runoff from precipitation normally drains by gravity into the stream upstream from the specified point. Values of drainage area given herein include all closed basins, or noncontributing areas, within the area.

Drainage basin: A part of the surface of the earth that is occupied by a drainage system, which consists of a surface stream or a body of impounded surface water together with all tributary surface streams and bodies of impounded surface water.

Hydrologic unit: A geographic area representing part or all of a surface drainage basin or distinct hydrologic feature as delineated by the office of Water Data Coordination on the State Hydrologic Unit Maps. Each hydrologic unit is identified by an eight-digit number.

Milligrams per liter: A unit for expressing the concentration of chemical constituents in solution. Milligrams per liter represents the mass of solute per unit volume (liter) of water. Concentration of suspended sediment also is expressed in milligrams per liter and is based on the mass of dry sediment per liter of water-sediment mixture.

Specific conductance: A measure of the ability of a water to conduct an electrical current. It is expressed in microsiemens per centimeter at 25 degrees Celsius. Specific conductance is related to the type and concentration of ions in solution and can be used for approximating the dissolved-solids concentration of the water. Commonly, the concentration of dissolved solids (in milligrams per liter) is about 65 percent of the specific conductance (in microsiemens). This relation is not constant from stream to stream and may vary in the same source with changes in the composition of the water. 
Tons per day: The quantity of substance in solution or suspension that passes a stream section during a 24 -hour period.

WATSTORE: Used as an abbreviation for the U.S. Geological Survey's National WATer-Data STOrage and REtrieval system, which was implemented by the U.S. Geological Survey in 1971 to provide for more efficient management of its data-releasing activities. WATSTORE is composed of a station header file, peak-flow file, daily-values file, water-quality file, and GroundWater Site-Inventory (GWSI) file.

\section{EXPLANATION OF HYDROLOGIC-DATA TABLES}

Surface- and ground-water data for the Jemez Mountains are presented separately. Summaries of data that were collected by the U.S. Geological Survey and retrieved through the WATSTORE system are presented in tables 1 and 2 (pages 10 and 31). Sources of data other than the U.S. Geological Survey also are identified. The locations of sites where data were collected are shown on plates 1 and 2 .

A list of 100 surface-water sites and peak-flow, water-quality, and daily-values data were retrieved from the WATSTORE header file, peak-flow file, water-quality file, and daily-values file and are listed by station in order of descending latitude and longitude in table 1. Period of record for available peak-flow data is listed. Summaries of water-quality data include the number of analyses and period of record available for major- and minorion, trace-element, radiochemical, biological, and sediment analyses. Summaries of daily values include beginning and ending year and month; number of days; number of missing days; number of water years; and maximum, minimum, and mean values for reservoir storage, water temperature, discharge, specific conductance, suspended-sediment concentration, suspended-sediment discharge, and rainfall accumulation.

\footnotetext{
Ground-water records for 294 sites including well data and the availability of water-quality and water-level data are summarized in table 2. Ground-water records, which include wells and springs, were retrieved from the WATSTORE header file and GWSI file. Well data were retrieved from the WATSTORE water-quality file and GWSI file. Water-level data are those data available in the GWSI file. Water-quality data, which also include wells and springs, were retrieved from the WATSTORE water-quality file. Summaries of water-quality data include number of analyses and period of record for available major- and minor-ion, trace-element, and radiochemical analyses. Ground-water stations are listed in order of ascending latitude and longitude.
} 


\section{REFERENCES CITED}

Abeyta, C.G., and Delaney, B.M., 1986, Annotated bibliography of the hydrology, geology, and geothermal research of the Jemez Mountains and vicinity, north-central New Mexico: U.S. Geological Survey Open-File Report 85-83, 155 p.

Goff, F.E., and others, 1982, Geochemical data for 95 thermal and non-thermal waters of the Valles Caldera, southern Jemez Mountains region, New Mexico: Los Alamos National Laboratory LA-9367-OBES, 51 p.

John, E.C., Enyart, Eugene, and Purtyman, W.D., 1967, Records of we11s, test holes, springs, and surface-water stations in the Los Alamos area, New Mexico: U.S. Geological Survey Open-File Report 67-123, 129 p.

Trainer, F.W., 1978, Geohydrologic data from the Jemez Mountains and vicinity, north-central New Mexico: U.S. Geological Survey Water-Resources Investigations $77-131,146 \mathrm{p}$.

U.S. Geological Survey, 1984, Water resources data--New Mexico, Water year 1983: U.S. Geological Survey Water-Data Report NM-83-1, 525 p.

\section{Fenton Hill series of Los Alamos National Laboratories reports:}

Purtymun, W.D., Adams, W.H., and Owens, J.W., 1975, Water quality in the vicinity of Fenton Hill Site, 1974: Los Alamos Scientific Laboratory LA-6093, $16 \mathrm{p}$.

Purtymun, W.D., Adams, W.H., and Stoker, A.K., 1978, Water quality in the vicinity of Fenton Hill Site, 1976: Los Alamos Scientific Laboratory Informa1 Report LA-7307-MS, $17 \mathrm{p}$.

Purtymun, W.D., Ferenbaugh, R.W., and Adams, W.H., 1981, Water quality in the vicinity of Fenton Hi11, 1980: Los Alamos Scientific Laboratory Progress Report LA-9007-PR, 16 p.

Purtymun, W.D., and others, 1976, Water quality in the vicinity of Fenton Hill Site, 1975: Los Alamos Scientific Laboratory Informal Report LA-6511-MS, $21 \mathrm{p}$.

1977, Water quality in the vicinity of Fenton Hill Site, 1977: Los Alamos Scientific Laboratory Progress Report LA-7468-PR, 21 p. 


\section{REPERENCES CITED-Concluded}

Purtymun, W.D., and others, 1980a, Water quality in the vicinity of Fenton Hill Site, 1978: Los Alamos Scientific Laboratory Progress Report LA-8217-PR, $21 \mathrm{p}$.

$1980 \mathrm{~b}$, Water quality in the vicinity of Fenton Hill, 1979: Los Alamos Scientific Laboratory Progress Report LA-8424-PR, 21 p.

1983, Water quality in the vicinity of Fenton Hill, 1981 and 1982: Los Alamos Scientific Laboratory Progress Report LA-9854-PR, 30 p. 
Table 1.--Sumary of surface-water data-collection sites in the Jenez Mountains, New Mexico

[Water-quality type of analysis: A, major and minor ion; $B$, trace element; $C$, radiochemical; D, biological; E, sediment. Parameter code: 00054, reservoir storage, in acre-feet; 00010, water temperature, in degrees Celsius; 00060, discharge, in cubic feet per second; 00095, specific conductance, in microsiemens per centimeter at 25 degrees Celsius; 80154, suspended-sediment concentration, in milligrams per liter; 80155, suspendedsediment discharge, in tons per day; 00045, rainfall accumulation, in inches. Statistic code: 30800, at 0800 hours; 32400 , at 2400 hours; 00011, once daily; 00003, mean daily; 00006, total (accunulative); 00001, maximum; 00002, minimum. Data source: USGS, U.S. Geological Survey; LA, LOS Alamos National Laboratories. --, no data]

\begin{tabular}{|c|c|c|c|c|c|c|c|c|}
\hline \multirow{3}{*}{ Number } & \multirow[b]{4}{*}{ Station } & \multirow[b]{4}{*}{ Station name } & \multirow[b]{4}{*}{$\begin{array}{l}\text { Lati- } \\
\text { tude }\end{array}$} & \multirow[b]{4}{*}{$\begin{array}{l}\text { Longi- } \\
\text { tude }\end{array}$} & \multirow[b]{4}{*}{ county } & \multicolumn{3}{|c|}{ Drain- } \\
\hline & & & & & & & age & Peak-flow \\
\hline & & & & & & Hydro- & area & period \\
\hline $\begin{array}{c}\text { on } \\
\text { plate } 1\end{array}$ & & & & & & $\begin{array}{l}\text { logic } \\
\text { unit }\end{array}$ & $\begin{array}{c}\text { (square } \\
\text { miles) }\end{array}$ & $\begin{array}{l}\text { of } \\
\text { record }\end{array}$ \\
\hline
\end{tabular}

108286700 Arroyo Seco near Abiquiu, N. Mex. 3616551062815 Rio Arriba $13020102 \quad 162 \quad 1953-64$

208286900 Abiquiu Reservoir near Abiquiu, $361424 \quad 1062544$ Rio Arriba $13020102 \quad 2,146 \quad$-N. Mex.

308287000 Rio Chama below Abiquiu Dam, $\quad 3614121062459$ Rio Arriba $13020102 \quad 2,147 \quad 1962-83$ N. Hex.

408287500 Rio Chama near Abiquiu, N. Mex. $\quad 361300 \quad 1061500$ Rio Arriba $13020102 \quad 2,284 \quad 1942-67$

5361212106202110 Arroyo Seco near Abiquiu, N. Mex. 3612121062021 Rio Arriba 13020102 --

6361208106205210 Barranca Ditch near Abiquiu, 3612081062052 Rio Arriba $13020102 \quad$-- $\quad$-N. Mex.

7361020106255510 Polvadera Creek near Cañones, 3610201062555 Rio Arriba N. Mex.

8360631106091010 Rio del Oso at Chili, N. Mex. 3606311060910 Rio Arriba 13020102

908289500 Chamita Ditch near Chamita, 3604571060654 Rio Arriba N. Mex. 08289800 Hernandez Ditch at Hernandez, N. Mex. 


\begin{tabular}{|c|c|c|c|c|c|c|c|c|c|c|c|c|c|}
\hline \multirow{2}{*}{\multicolumn{3}{|c|}{ Water-quality data }} & \multicolumn{11}{|c|}{ Daily-values data } \\
\hline & & & \multirow[b]{2}{*}{$\begin{array}{l}\text { Param- } \\
\text { eter } \\
\text { code }\end{array}$} & \multirow[b]{2}{*}{$\begin{array}{l}\text { Sta- } \\
\text { tistic } \\
\text { code }\end{array}$} & & \multicolumn{2}{|r|}{ Number } & & \multirow[b]{2}{*}{ Minimum } & \multirow[b]{2}{*}{ Mean } & \multirow{2}{*}{$\begin{array}{l}\text { Data } \\
\text { source/ } \\
\text { site } \\
\text { name }\end{array}$} \\
\hline $\begin{array}{c}\text { Type } \\
\text { of } \\
\text { analysis }\end{array}$ & $\begin{array}{c}\text { Number } \\
\text { of } \\
\text { analyses }\end{array}$ & $\begin{array}{l}\text { Period } \\
\text { of } \\
\text { record }\end{array}$ & & & 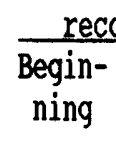 & $\frac{\text { cord }}{\text { Ending }}$ & $\begin{array}{c}\text { Number } \\
\text { of } \\
\text { days }\end{array}$ & $\begin{array}{l}\text { of } \\
\text { missing } \\
\text { days }\end{array}$ & $\begin{array}{l}\text { Wat } \\
\text { yea. }\end{array}$ & $\begin{array}{l}\text { er } \\
\text { rs Maximum }\end{array}$ & & & \\
\hline- & - & -- & -- & - & $\cdots$ & $\cdots$ & - & - & - & - & -- & - & - \\
\hline D & 2 & 1975 & $\begin{array}{l}00054 \\
00054\end{array}$ & $\begin{array}{l}30800 \\
32400\end{array}$ & $\begin{array}{l}10-69 \\
10-65 \\
01-76 \\
03-76\end{array}$ & $\begin{array}{l}12-75 \\
09-69 \\
01-76 \\
09-83\end{array}$ & $\begin{array}{r}2,283 \\
1,461 \\
31 \\
2,770\end{array}$ & $\begin{array}{l}0 \\
0 \\
0 \\
0\end{array}$ & $\begin{array}{l}7 \\
4 \\
1 \\
7\end{array}$ & $\begin{array}{r}205,300.00 \\
99,050.00 \\
3,220.00 \\
198,300.00\end{array}$ & $\begin{array}{r}281.00 \\
.00 \\
.00 \\
.00\end{array}$ & $\begin{array}{r}17,332.00 \\
12,369.00 \\
900.35 \\
51,980.00\end{array}$ & - \\
\hline E & $178 \quad 15$ & $962-83$ & $\begin{array}{l}00010 \\
00060 \\
00095 \\
80154 \\
80155\end{array}$ & $\begin{array}{l}00011 \\
00003 \\
00011 \\
00003 \\
00003\end{array}$ & $\begin{array}{l}10-62 \\
10-69 \\
11-61 \\
10-69 \\
10-62 \\
10-62\end{array}$ & $\begin{array}{l}09-68 \\
11-74 \\
09-83 \\
10-74 \\
12-74 \\
12-74\end{array}$ & $\begin{array}{l}1,754 \\
1,040 \\
8,004 \\
1,003 \\
4,383 \\
4,475\end{array}$ & $\begin{array}{r}438 \\
847 \\
0 \\
854 \\
92 \\
0\end{array}$ & $\begin{array}{r}6 \\
6 \\
22 \\
6 \\
13 \\
13\end{array}$ & $\begin{array}{r}54.99 \\
26.09 \\
2,780.00 \\
1,220.00 \\
85,000.00 \\
214,000.00\end{array}$ & $\begin{array}{r}.00 \\
.00 \\
8.80 \\
146.00 \\
.00 \\
.00\end{array}$ & $\begin{array}{r}10.76 \\
10.70 \\
438.80 \\
469.14 \\
1,619.74 \\
1,185.80\end{array}$ & -- \\
\hline-- & -- & -- & $\begin{array}{l}00060 \\
80154 \\
80155\end{array}$ & $\begin{array}{l}00003 \\
00003 \\
00003\end{array}$ & $\begin{array}{l}10-41 \\
10-55 \\
10-55\end{array}$ & $\begin{array}{l}09-67 \\
06-56 \\
06-56\end{array}$ & $\begin{array}{r}9,496 \\
264 \\
274\end{array}$ & $\begin{array}{r}0 \\
10 \\
0\end{array}$ & $\begin{array}{r}26 \\
1 \\
1\end{array}$ & $\begin{array}{l}5,330.00 \\
6,700.00 \\
9,070.00\end{array}$ & $\begin{array}{r}1.00 \\
18.00 \\
1.00\end{array}$ & $\begin{array}{l}397.09 \\
664.87 \\
792.13\end{array}$ & - \\
\hline$A, B$ & 1 & 1974 & -- & -- & $\cdots$ & $\cdots$ & - & -- & -- & $\cdots$ & - & -- & $\cdots$ \\
\hline$A, B$ & 1 & 1974 & -- & -- & -- & $-\cdots$ & -- & - & - & -- & -- & -- & -- \\
\hline$A, B$ & 1 & 1982 & -- & -- & $--\cdot$ & -- & - & -- & $\cdots$ & -- & -- & - & -- \\
\hline$A, B$ & 1 & 1974 & - & -- & $\cdots$ & -- & - & - & -- & -- & -- & -- & -- \\
\hline-- & -- & -- & 00060 & 00003 & $\begin{array}{l}10-64 \\
06-67\end{array}$ & $\begin{array}{l}03-67 \\
09-68\end{array}$ & $\begin{array}{l}912 \\
488\end{array}$ & $\begin{array}{l}0 \\
0\end{array}$ & $\begin{array}{l}3 \\
1\end{array}$ & $\begin{array}{l}19.00 \\
21.00\end{array}$ & $\begin{array}{l}.00 \\
.00\end{array}$ & $\begin{array}{l}4.59 \\
6.11\end{array}$ & -- \\
\hline- & -- & -- & 00060 & 00003 & $04-63$ & $09-68$ & 2,010 & 0 & 6 & 46.00 & .00 & 8.33 & -- \\
\hline
\end{tabular}


Table 1. - - Sumary of surface-water data-collection sites in the Jenez Mountains, Nev Hexico--Continued

\begin{tabular}{|c|c|c|c|c|c|c|c|c|}
\hline $\begin{array}{l}\text { Number } \\
\text { on } \\
\text { plate }\end{array}$ & $\begin{array}{l}\text { Station } \\
\text { number }\end{array}$ & Station name & $\begin{array}{l}\text { Lati- } \\
\text { tude }\end{array}$ & $\begin{array}{l}\text { Longi- } \\
\text { tude }\end{array}$ & County & $\begin{array}{c}\text { Hydro- } \\
\text { logic } \\
\text { unit }\end{array}$ & $\begin{array}{l}\text { Drain- } \\
\text { age } \\
\text { area } \\
\text { (square } \\
\text { miles) }\end{array}$ & $\begin{array}{c}\text { Peak-flow } \\
\text { period } \\
\text { of } \\
\text { record }\end{array}$ \\
\hline 11 & 08290000 & Rio Chama near Chamita, N. Mex. & 360426 & 1060640 & Rio Arriba & 13020102 & 3,144 & $1915-83$ \\
\hline 12 & 08280700 & $\begin{array}{l}\text { Guique Ditch near San Juan } \\
\text { Pueblo, N. Kex. }\end{array}$ & 360416 & 1060442 & Rio Arriba & 13020101 & - & - \\
\hline 13 & 08280100 & $\begin{array}{l}\text { San Juan Lat above San Juan } \\
\text { Pueblo, N. Hex. }\end{array}$ & 360403 & 1060407 & Rio Arriba & 13020101 & -- & -- \\
\hline 14 & 08281100 & $\begin{array}{l}\text { Rio Grande above San Juan Pueblo, } \\
\text { N. Mex. }\end{array}$ & 360358 & 1060434 & Rio Arriba & 13020101 & 10,550 & $1963-83$ \\
\hline 15 & 08280200 & $\begin{array}{l}\text { San Juan Pueblo Ditch above San } \\
\text { Juan Pueblo, N. Mex. }\end{array}$ & 360355 & 1060410 & Rio Arriba & 13020101 & -- & - \\
\hline 16 & 08321900 & $\begin{array}{l}\text { Rio de Las Vacas near Señorita, } \\
\text { N. Mex. }\end{array}$ & 355935 & 1064745 & Sandoval & 13020202 & 26.8 & $1957-83$ \\
\hline 17 & 08292000 & $\begin{array}{l}\text { Santa Clara Creek near Española, } \\
\text { N. Mex. }\end{array}$ & 355840 & 1061020 & Rio Arriba & 13020101 & 34.5 & $1936-70$ \\
\hline 18 & 355820106052010 & $\begin{array}{l}\text { Santa Clara C Hwy } 30 \text { near } \\
\text { Española, N. Mex. }\end{array}$ & 355820 & 1060520 & Rio Arriba & 13020101 & -- & -- \\
\hline 19 & 08319500 & $\begin{array}{l}\text { San Antonio Creek near Los } \\
\text { Alamos, N. Hex. }\end{array}$ & 355745 & 1062925 & Sandoval & 13020202 & -- & - \\
\hline 20 & 355714106472110 & 20N.01E.24.121 Rio de Las Vacas & 355714 & 1064721 & Sandoval & -- & -- & -- \\
\hline 213 & 355657106382210 & 20N.03E.20.311 San Antonio Creek & 355657 & 1063822 & Sandoval & - & -- & - \\
\hline
\end{tabular}




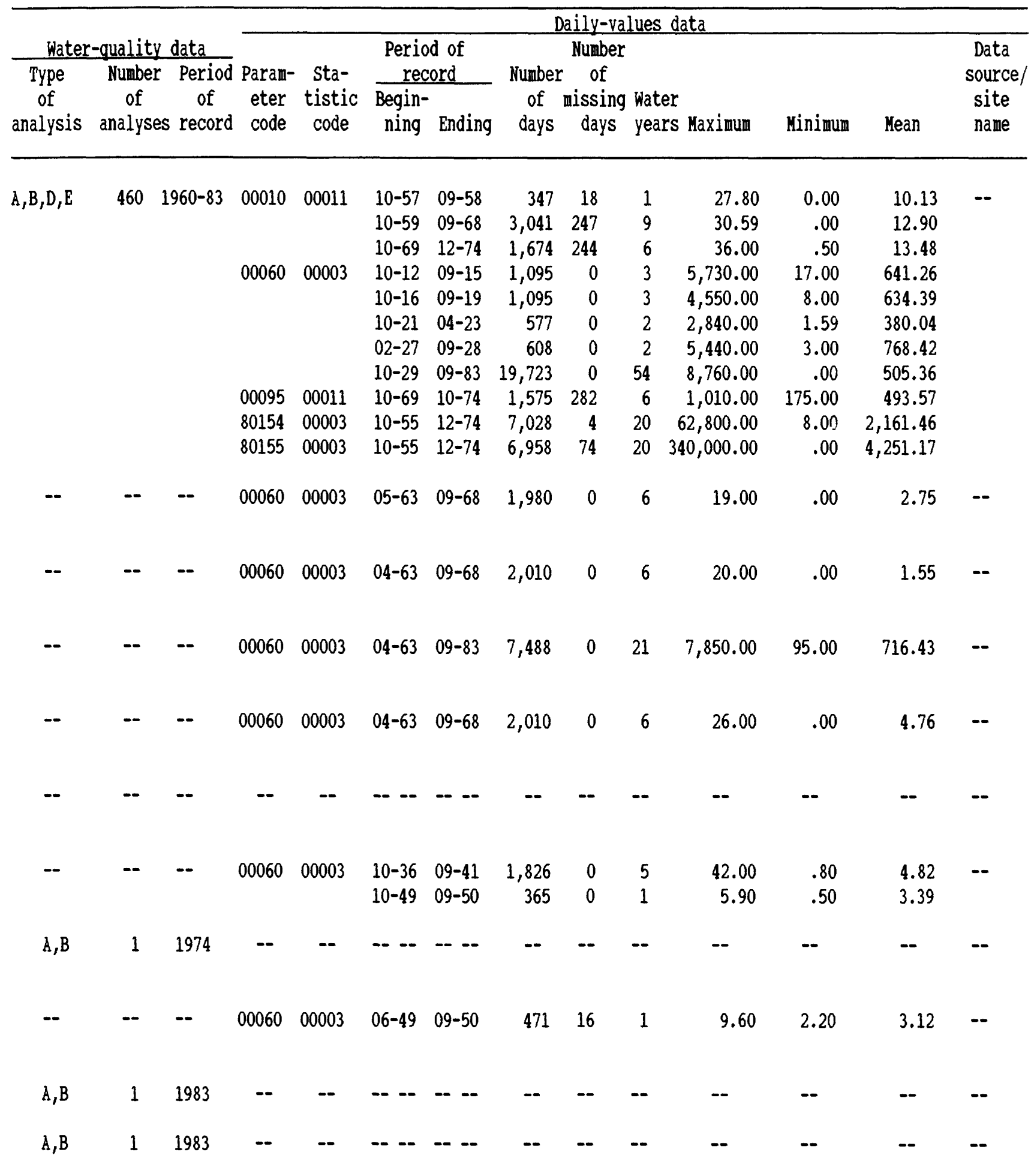


Table 1.--Sumary of surface-rater data-collection sites in the Jenez Hountains, Mev Mexico--Continued

\begin{tabular}{|c|c|c|c|c|c|c|c|c|c|}
\hline $\begin{array}{l}\text { Numbe } \\
\text { on } \\
\text { plate }\end{array}$ & & $\begin{array}{l}\text { Station } \\
\text { number }\end{array}$ & Station name & $\begin{array}{l}\text { Lati- } \\
\text { tude }\end{array}$ & $\begin{array}{l}\text { Longi- } \\
\text { tude }\end{array}$ & county & $\begin{array}{l}\text { Hydro- } \\
\text { logic } \\
\text { unit }\end{array}$ & $\begin{array}{l}\text { Drain- } \\
\text { age } \\
\text { area } \\
\text { (square } \\
\text { miles) }\end{array}$ & $\begin{array}{l}\text { Peak-flow } \\
\text { period } \\
\text { of } \\
\text { record }\end{array}$ \\
\hline 22 & & 08332700 & $\begin{array}{l}\text { San Pablo Canyon near Cuba, } \\
\text { N. Hex. }\end{array}$ & 355655 & 1065644 & Sandoval & 13020204 & 12.8 & 3 $1971-82$ \\
\hline 23 & & 5408106371110 & Sulphur Creek & 355408 & 1063711 & Sandoval & - & - & -- \\
\hline 24 & & 5321106180810 & Pueblo 1 & 355321 & 1061808 & Los Alamos & -- & -- & - \\
\hline 25 & & 5317106181210 & Acid Weir & 355317 & 1061812 & Los Alamos & - & - & - \\
\hline 26 & & 5306106161210 & Pueblo 2 & 355306 & 1061612 & Los Alamos & -- & - & -- \\
\hline 27 & & 5239106375410 & $\begin{array}{l}\text { Sulphur Creek above Redondo } \\
\text { Creek, N. Mex. }\end{array}$ & 355239 & 1063754 & Sandoval & 13020202 & -- & -- \\
\hline 283 & 3552 & 236106374210 & $\begin{array}{l}\text { Redondo Creek above Sulphur Creek, } \\
\text { N. Mex. }\end{array}$ & 355236 & 1063742 & Sandoval & 13020202 & - & -- \\
\hline 29 & & 08319945 & $\begin{array}{l}\text { Redondo Creek near Jemez Springs, } \\
\text { N. Hex. }\end{array}$ & 355234 & 1063750 & Sandoval & 13020202 & - & -- \\
\hline 30 & 355 & 5232106132610 & Pueblo 3 & 355232 & 1061326 & Santa Fe & 13020202 & -- & $m$ \\
\hline
\end{tabular}




\begin{tabular}{|c|c|c|c|c|c|c|c|c|c|c|c|c|c|}
\hline \multirow{2}{*}{\multicolumn{3}{|c|}{ Water-quality data }} & \multicolumn{11}{|c|}{ Daily-values data } \\
\hline & & & \multirow[b]{2}{*}{$\begin{array}{l}\text { Param- } \\
\text { eter } \\
\text { code }\end{array}$} & \multirow[b]{2}{*}{$\begin{array}{l}\text { Sta- } \\
\text { tistic } \\
\text { code }\end{array}$} & & \multicolumn{3}{|c|}{ Number } & \multirow[b]{2}{*}{ Maximum } & \multirow[b]{2}{*}{ Minimum } & \multirow[b]{2}{*}{ Mean } & \multirow{2}{*}{$\begin{array}{c}\text { Data } \\
\text { source } \\
\text { site } \\
\text { name }\end{array}$} \\
\hline $\begin{array}{c}\text { Type } \\
\text { of } \\
\text { analysis }\end{array}$ & $\begin{array}{c}\text { Number } \\
\text { of } \\
\text { analyses }\end{array}$ & $\begin{array}{l}\text { Period } \\
\text { of } \\
\text { s record }\end{array}$ & & & $\begin{array}{c}\text { rec } \\
\text { Begin- } \\
\text { ning }\end{array}$ & Ending & $\begin{array}{c}\text { Number } \\
\text { of } \\
\text { days }\end{array}$ & $\begin{array}{c}\text { of } \\
\text { missing } \\
\text { days }\end{array}$ & $\begin{array}{l}\text { Water } \\
\text { years }\end{array}$ & & & & \\
\hline \multirow[t]{4}{*}{$A, B, E$} & 22 & $1979-82$ & 00045 & 00006 & $10-69$ & $09-76$ & 2,557 & 0 & 7 & 2.26 & 0.00 & 0.01 & -- \\
\hline & & & & & $04-79$ & $03-80$ & 248 & 118 & 2 & 5.72 & .00 & .06 & \\
\hline & & & & & $06-80$ & $09-82$ & 833 & 19 & 2 & 2.18 & .00 & .03 & \\
\hline & & & 00060 & 00003 & $04-79$ & $10-82$ & 1,273 & 37 & 5 & 33.00 & .00 & .86 & \\
\hline$A, B$ & 1 & 1983 & -- & -- & -- -- & -- -- & -- & -- & -- & -- & -- & -- & -- \\
\hline$A, B$ & 52 & $1953-59$ & -- & -- & -- & --- & -- & -- & -- & -- & -- & -- & USGS/-- \\
\hline A & 391 & $1953-61$ & -- & -- & --- & ---- & - & -- & -- & - & - & -- & USGS/-- \\
\hline A & 44 & $1948-59$ & -- & -- & --- & ---- & -- & -- & -- & -- & -- & -- & USGS/-- \\
\hline$A, C$ & 4 & $1974-75$ & -- & -- & --- & --- & -- & -- & -- & -- & -- & -- & LA/Site V \\
\hline$A, C$ & 41 & $1974-75$ & -- & -- & --- & --- & -- & -- & -- & -- & -- & -- & LA/Site 0 \\
\hline$A, B$ & 1 & 1982 & 00060 & 00003 & $11-81$ & $09-83$ & 690 & 9 & 2 & 15.00 & .18 & 1.86 & -- \\
\hline A & 151 & $1957-59$ & -- & -- & --- & --- & -- & -- & -- & -- & -- & -- & USGS/-- \\
\hline
\end{tabular}


Table 1.--Sumary of surface-water data-collection sites in the Jenez Hountains, Her Mexico--Continued

\begin{tabular}{|c|c|c|c|c|c|c|c|c|}
\hline $\begin{array}{l}\text { Number } \\
\text { on } \\
\text { plate } 1\end{array}$ & $\begin{array}{l}\text { Station } \\
\text { number }\end{array}$ & Station name & $\begin{array}{l}\text { Lati- } \\
\text { tude }\end{array}$ & $\begin{array}{l}\text { Longi- } \\
\text { tude }\end{array}$ & County & $\begin{array}{l}\text { Hydro- } \\
\text { logic } \\
\text { unit }\end{array}$ & $\begin{array}{c}\text { Drain- } \\
\text { age } \\
\text { area } \\
\text { (square } \\
\text { miles) }\end{array}$ & $\begin{array}{l}\text { Peak-flow } \\
\text { period } \\
\text { of } \\
\text { record }\end{array}$ \\
\hline 31 & 08313000 & $\begin{array}{l}\text { Rio Grande at otowi Bridge, } \\
\text { N. Mex. }\end{array}$ & 355229 & 1060830 & Santa Fe & 13020101 & 14,300 & $1895-1983$ \\
\hline
\end{tabular}

08319950 Sulfur Creek near Jenez Springs,

3552141063817 Sandoval

13020202

N. Hex.

33355118106381910 San Antonio Creek above Sulphur

3552131063819 Sandoval 13020202 Creek, N. Mex.

34355118106381810 Sulphur Creek at La Cueva,

$355213 \quad 1063818$ Sandoval 13020202 N. Mex.

$355213 \quad 1063818 \quad$ Sandoval 13020202

355118106381800 Sulphur Creek at La Cueva, N. Mex.

$355200 \quad 1062710$ Sandoval 13020202

08320500 East Fork Jemez River near Los Alanos, N. Mex. 


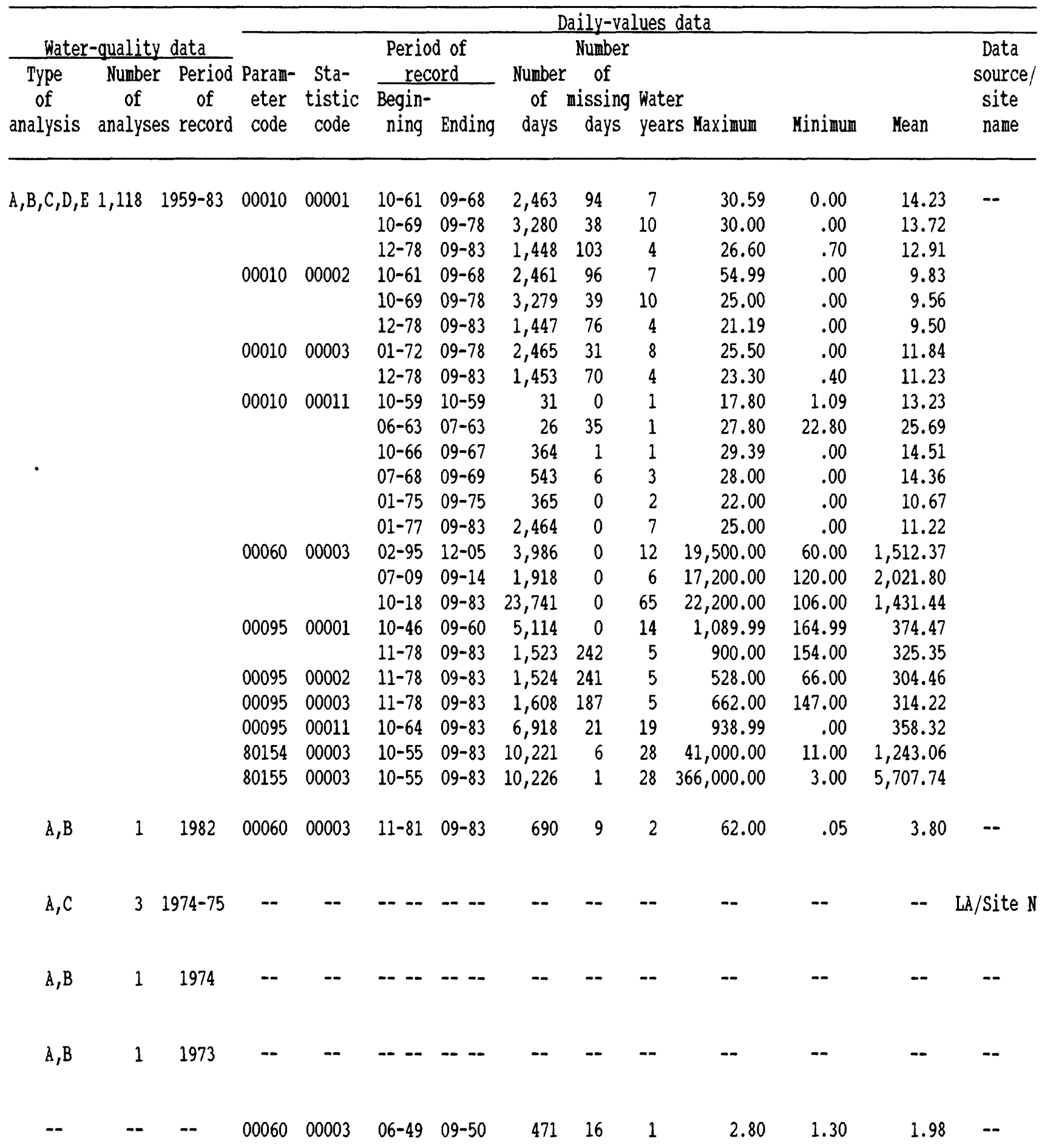


Table 1.--Sumary of surface-water data-collection sites in the Jenez Hountains, Hev Mexico--Continued

\begin{tabular}{|c|c|c|c|c|c|c|c|c|}
\hline $\begin{array}{l}\text { Number } \\
\text { on } \\
\text { plate }\end{array}$ & $\begin{array}{l}\text { Station } \\
\text { number }\end{array}$ & Station name & $\begin{array}{l}\text { Lati- } \\
\text { tude }\end{array}$ & $\begin{array}{l}\text { Longi- } \\
\text { tude }\end{array}$ & County & $\begin{array}{l}\text { Hydro- } \\
\text { logic } \\
\text { unit }\end{array}$ & $\begin{array}{c}\text { Drain- } \\
\text { age } \\
\text { area } \\
\text { (square } \\
\text { miles) }\end{array}$ & $\begin{array}{l}\text { Peak-flon } \\
\text { period } \\
\text { of } \\
\text { record }\end{array}$ \\
\hline 37 & 354941106383810 & $\begin{array}{l}\text { East Fork Jemez River above San } \\
\text { Antonio Creek, N. Mex. }\end{array}$ & 354941 & 1063838 & Sandoval & 13020202 & -- & -- \\
\hline 38 & 08321500 & $\begin{array}{l}\text { Jemez River below East Fork near } \\
\text { Jemez Springs, N. Hex. }\end{array}$ & 354939 & 1063851 & Sandoval & 13020202 & 173 & $1959-83$ \\
\hline 39 & 354855106312410 & East Fork Jemez River - Tributary & 354855 & 1063124 & Sandoval & -- & -- & - \\
\hline 40 & 08313300 & $\begin{array}{l}\text { Rito de Los Frijoles near Los } \\
\text { Alamos, N. Mex. }\end{array}$ & 354855 & 1062135 & Los Alamos & 13020201 & 8.9 & -- \\
\hline 41 & 354721106411310 & $\begin{array}{l}\text { Jemez River belori Soda Dam, } \\
\text { N. Mex. }\end{array}$ & 354721 & 1064113 & Sandoval & 13020202 & -- & $\therefore$ \\
\hline 42 & 08313350 & $\begin{array}{l}\text { Rito de Los Frijoles in Bandelier } \\
\text { National Monument, N. Mex. }\end{array}$ & 354708 & 1061650 & Sandoval & 13020201 & 17.5 & $1965-83$ \\
\hline 43 & 354706106321010 & Vallecito Creek & 354706 & 1063210 & Sandoval & -- & - & - \\
\hline 44 & 354635106260410 & $\begin{array}{l}\text { 18N.05E.20.34411 Cochiti Creek } \\
0.8 \text { mile south of Tent Rock, } \\
\text { N. Mex. }\end{array}$ & 354635 & 106260 & Sandoval & -- & -- & - \\
\hline 45 & 354610106413210 & $\begin{array}{l}\text { Jemez River below Jemez Springs, } \\
\text { N. Mex. }\end{array}$ & 354610 & 1064132 & Sandoval & 13020202 & -- & -- \\
\hline 46 & 354605106413610 & $\begin{array}{l}\text { Jemez River at Jemez Springs, } \\
\text { N. Mex. }\end{array}$ & 354605 & 1064136 & Sandoval & 13020202 & -- & -- \\
\hline 47 & 354526106274910 & $\begin{array}{l}\text { 18N.04E.25.443 Bland Creek at } \\
\text { Bland, N. Mex. }\end{array}$ & 354526 & 1062749 & Sandoval & -- & -- & -- \\
\hline 48 & 08323000 & $\begin{array}{l}\text { Rio Guadalupe at Box Canyon near } \\
\text { Jemez, N. Mex. }\end{array}$ & 354352 & 1064544 & Sandoval & 13020202 & 235 & $1939-83$ \\
\hline 49 & 08313400 & $\begin{array}{l}\text { Bland Canyon near Cochiti, } \\
\text { N. Mex. }\end{array}$ & 354211 & 1062456 & Sandoval & 13020201 & 7.57 & $71962-83$ \\
\hline 50 & 08323500 & $\begin{array}{l}\text { Rio Guadalupe near Jemez Springs, } \\
\text { N. Hex. }\end{array}$ & 354210 & 1064515 & Sandoval & 13020202 & 230 & -- \\
\hline
\end{tabular}




\begin{tabular}{|c|c|c|c|c|c|c|c|c|c|c|c|c|c|}
\hline \multirow{2}{*}{\multicolumn{3}{|c|}{ Water-quality data }} & \multicolumn{11}{|c|}{ Daily-values data } \\
\hline & & & \multirow[b]{2}{*}{$\begin{array}{l}\text { Param- } \\
\text { eter } \\
\text { code }\end{array}$} & \multirow[b]{2}{*}{$\begin{array}{l}\text { Sta- } \\
\text { tistic } \\
\text { code }\end{array}$} & & \multirow{2}{*}{$\begin{array}{c}\text { Number } \\
\text { of } \\
\text { days }\end{array}$} & & \multirow{2}{*}{ Water } & \multirow[b]{2}{*}{ Maximum } & \multirow[b]{2}{*}{ Minimum } & \multirow[b]{2}{*}{ Kean } & \multirow{2}{*}{$\begin{array}{l}\text { Data } \\
\text { source/ } \\
\text { site } \\
\text { name }\end{array}$} \\
\hline $\begin{array}{c}\text { Type } \\
\text { of } \\
\text { analysis }\end{array}$ & $\begin{array}{l}\text { Number } \\
\text { of } \\
\text { analyses }\end{array}$ & $\begin{array}{l}\text { Period } \\
\text { of } \\
\text { s record }\end{array}$ & & & $\begin{array}{l}\text { rece } \\
\begin{array}{c}\text { Begin- } \\
\text { ning }\end{array}\end{array}$ & $\frac{\text { ord }}{\text { Ending }}$ & & $\begin{array}{c}\text { of } \\
\text { missing } \\
\text { days }\end{array}$ & & & & & \\
\hline c & 1 & 1974 & -- & -- & --- & --- & -- & -- & -- & -- & -- & -- & -- \\
\hline$A, B, C$ & 661 & $1963-76$ & 00060 & 00003 & $\begin{array}{l}03-58 \\
07-81\end{array}$ & $\begin{array}{l}09-76 \\
09-83\end{array}$ & $\begin{array}{r}6,789 \\
808\end{array}$ & $\begin{array}{r}0 \\
14\end{array}$ & $\begin{array}{r}19 \\
3\end{array}$ & $\begin{array}{r}1,450.00 \\
767.00\end{array}$ & $\begin{array}{l}3.30 \\
9.00\end{array}$ & $\begin{array}{l}30.81 \\
41.87\end{array}$ & $\mathrm{LA} /$ Site J \\
\hline$A, B$ & 1 & 1983 & -- & -- & --- & --- & -- & -- & -- & -- & -- & -- & -- \\
\hline-- & -- & -- & 00060 & 00003 & $10-60$ & $10-63$ & 1,126 & 0 & 4 & 9.00 & .69 & 1.57 & -- \\
\hline A & 1 & 1975 & -- & -- & --- & -- & -- & -- & - & -- & -- & -- & -- \\
\hline E & 1641 & $1977-81$ & 00060 & 00003 & $\begin{array}{l}08-63 \\
07-77\end{array}$ & $\begin{array}{l}09-69 \\
11-82\end{array}$ & $\begin{array}{l}2,253 \\
1,960\end{array}$ & $\begin{array}{r}0 \\
19\end{array}$ & $\begin{array}{l}7 \\
7\end{array}$ & $\begin{array}{r}7.80 \\
80.00\end{array}$ & $\begin{array}{l}.19 \\
.08\end{array}$ & $\begin{array}{l}1.16 \\
1.78\end{array}$ & -- \\
\hline$A, B$ & 1 & 1983 & -- & -- & --- & --- & -- & - & -- & -- & -- & -- & -- \\
\hline A & 1 & 1982 & -- & -- & ---- & --- & -- & -- & -- & -- & -- & -- & -- \\
\hline A & 2 & 1975 & -- & -- & -- -- & --- & -- & -- & -- & -- & -- & -- & -- \\
\hline$A, B$ & 1 & 1974 & -- & -- & -- & $\cdots$ & - & - & - & -- & -- & -- & -- \\
\hline$A, B$ & 1 & 1983 & -- & -- & -- & --- & - & -- & -- & -- & -- & -- & -- \\
\hline$A, B, C$ & $12 \quad 19$ & $1963-76$ & 00060 & 00003 & $\begin{array}{l}10-58 \\
07-81\end{array}$ & $\begin{array}{l}09-76 \\
09-83\end{array}$ & $\begin{array}{r}6,575 \\
808\end{array}$ & $\begin{array}{r}0 \\
14\end{array}$ & $\begin{array}{r}18 \\
3\end{array}$ & $\begin{array}{r}1,350.00 \\
626.00\end{array}$ & $\begin{array}{l}3.40 \\
4.00\end{array}$ & $\begin{array}{l}35.45 \\
58.80\end{array}$ & -- \\
\hline-- & -- & -- & 00045 & 00006 & $10-68$ & $09-76$ & 2,922 & 0 & 8 & 1.73 & .00 & .02 & -- \\
\hline-- & -- & -- & 00060 & 00003 & $\begin{array}{l}10-38 \\
10-49\end{array}$ & $\begin{array}{l}09-42 \\
09-50\end{array}$ & $\begin{array}{r}1,461 \\
365\end{array}$ & $\begin{array}{l}0 \\
0\end{array}$ & $\begin{array}{l}4 \\
1\end{array}$ & $\begin{array}{r}2,100.00 \\
109.00\end{array}$ & $\begin{array}{l}4.19 \\
3.09\end{array}$ & $\begin{array}{l}85.52 \\
14.18\end{array}$ & -- \\
\hline
\end{tabular}


Table 1.--Sumary of surface-water data-collection sites in the Jenez Mountains, Ner Mexico--Continued

\begin{tabular}{|c|c|c|c|c|c|c|c|c|}
\hline $\begin{array}{l}\text { Number } \\
\text { on } \\
\text { plate 1 }\end{array}$ & $\begin{array}{l}\text { Station } \\
\text { number }\end{array}$ & Station name & $\begin{array}{l}\text { Lati- } \\
\text { tude }\end{array}$ & $\begin{array}{l}\text { Longi- } \\
\text { tude }\end{array}$ & County & $\begin{array}{l}\text { Hydro- } \\
\text { logic } \\
\text { unit }\end{array}$ & $\begin{array}{l}\text { Drain- } \\
\text { age } \\
\text { area } \\
\text { (square } \\
\text { miles) }\end{array}$ & $\begin{array}{l}\text { Peak-flow } \\
\text { period } \\
\text { of } \\
\text { record }\end{array}$ \\
\hline 513 & 354014106443300 & $\begin{array}{l}\text { Jemez River above Rio Guadalupe } \\
\text { Canyonland, N. Hex. }\end{array}$ & 354014 & 1064433 & Sandoval & 13020202 & -- & -- \\
\hline 523 & 354013106443500 & $\begin{array}{l}\text { Rio Guadalupe above Jemez River } \\
\text { Canyonland, N. Hex. }\end{array}$ & 354013 & 1064435 & Sandoval & 13020202 & -- & -- \\
\hline 53 & 08324000 & Jemez River near Jemez, N. Mex. & 353942 & 1064434 & Sandoval & 13020202 & 470 & $1936-83$ \\
\hline 54 & 08317300 & $\begin{array}{l}\text { Cochiti Lake near Cochiti } \\
\text { Pueblo, N. Mex. }\end{array}$ & 353811 & 1061905 & Sandoval & 13020201 & 14,900 & -- \\
\hline 55 & 08314000 & $\begin{array}{l}\text { Sili Main Canal (at head) at } \\
\text { Cochiti, N. Mex. }\end{array}$ & 353710 & 1061928 & Sandoval & 13020201 & -- & -- \\
\hline 56 & 08314500 & Rio Grande at Cochiti, N. Mex. & 353710 & 1061908 & Sandoval & 13020201 & -- & $1927-70$ \\
\hline 57 & 08900100 & $\begin{array}{l}\text { Rio Grande Cochiti to Isleta } \\
\text { X-Section 1, N. Mex. }\end{array}$ & 353709 & 1061945 & Sandoval & 13020201 & -- & -- \\
\hline 58 & 08317400 & $\begin{array}{l}\text { Rio Grande beloi Cochiti Dam, } \\
\text { N. Hex. }\end{array}$ & 353704 & 1061926 & Sandoval & 13020201 & 14,900 & $1971-83$ \\
\hline 59 & 08313500 & $\begin{array}{l}\text { Cochiti East Side Main Canal at } \\
\text { Cochiti, N. Kex. }\end{array}$ & 353702 & 1061926 & Sandoval & 13020201 & -- & -- \\
\hline 60 & 08900200 & $\begin{array}{l}\text { Rio Grande Cochiti to Isleta } \\
\text { X-Section } 2, N \text {. Mex. }\end{array}$ & 353616 & 1062010 & Sandoval & 13020201 & -- & -- \\
\hline 61 & 08900300 & $\begin{array}{l}\text { Rio Grande Cochiti to Isleta } \\
\text { X-Section 3, N. Mex. }\end{array}$ & 353539 & 1062113 & Sandoval & 13020201 & -- & -- \\
\hline $62 \quad 35$ & 353522106531610 & $\begin{array}{l}\text { Cuchillo Arroyo Hixy } 44 \text { San } \\
\text { Ysidro, N. Mex. }\end{array}$ & 353522 & 1065316 & Sandoval & 13020202 & -- & -- \\
\hline
\end{tabular}




\begin{tabular}{|c|c|c|c|c|c|c|c|c|c|c|c|c|c|}
\hline \multirow{2}{*}{\multicolumn{3}{|c|}{ Water-quality data }} & \multicolumn{11}{|c|}{ Daily-values data } \\
\hline & & & \multirow[b]{2}{*}{$\begin{array}{l}\text { Param- } \\
\text { eter } \\
\text { code }\end{array}$} & \multirow[b]{2}{*}{$\begin{array}{l}\text { Sta- } \\
\text { tistic } \\
\text { code }\end{array}$} & & \multicolumn{2}{|c|}{ Number } & \multirow{2}{*}{\multicolumn{2}{|c|}{$\begin{array}{l}\text { Water } \\
\text { years Maximum }\end{array}$}} & \multirow[b]{2}{*}{ Minimum } & \multirow[b]{2}{*}{ Mean } & \multirow{2}{*}{$\begin{array}{l}\text { Data } \\
\text { source, } \\
\text { site } \\
\text { name }\end{array}$} \\
\hline $\begin{array}{c}\text { Type } \\
\text { of } \\
\text { analysis }\end{array}$ & $\begin{array}{c}\text { Number } \\
\text { of } \\
\text { analyses }\end{array}$ & $\begin{array}{l}\text { Period } \\
\text { of } \\
\text { s record }\end{array}$ & & & $\begin{array}{l}\frac{\text { reco }}{\text { Begin- }} \\
\text { ning }\end{array}$ & Ending & $\begin{array}{c}\text { Number } \\
\text { of } \\
\text { days }\end{array}$ & $\begin{array}{l}\text { of } \\
\text { missing } \\
\text { days }\end{array}$ & & & & & \\
\hline$A, B$ & 1 & 1973 & -- & -- & --- & -- -- & -- & -- & -- & -- & -- & -- & -- \\
\hline$A, B$ & 1 & 1973 & -- & -- & -- & -- -- & -- & -- & - & -- & -- & - & LA/Site $Q$ \\
\hline$A, B, C, E$ & 421 & $1974-83$ & 00060 & 00003 & $\begin{array}{l}10-36 \\
10-49 \\
03-53\end{array}$ & $\begin{array}{l}04-41 \\
09-50 \\
09-83\end{array}$ & $\begin{array}{r}1,673 \\
365 \\
11,171\end{array}$ & $\begin{array}{l}0 \\
0 \\
0\end{array}$ & $\begin{array}{r}5 \\
1 \\
31\end{array}$ & $\begin{array}{r}2,240.00 \\
133.00 \\
3,160.00\end{array}$ & $\begin{array}{r}12.00 \\
11.00 \\
2.10\end{array}$ & $\begin{array}{l}85.50 \\
31.70 \\
71.43\end{array}$ & -- \\
\hline$A, B, C, D$ & 451 & $1981-83$ & 00054 & 32400 & $01-73$ & $09-83$ & 3,925 & 0 & 11 & $184,400.00$ & .00 & $39,038.00$ & -- \\
\hline-- & -- & -- & 00060 & 00003 & $08-54$ & $09-83$ & 10,653 & 0 & 30 & 84.00 & .00 & 26.62 & -- \\
\hline$E$ & 5 & 1973 & 00060 & 00003 & $06-26$ & $10-70$ & 16,223 & 1 & 46 & $22,400.00$ & 1.00 & $1,298.40$ & -- \\
\hline E & 71 & $1970-80$ & -- & -- & -. - & -- & -- & - & - & -- & - & - & - \\
\hline$C, D, E$ & $34 \quad 1$ & $1971-82$ & $\begin{array}{l}00010 \\
00010 \\
00010 \\
00060 \\
00095 \\
80154 \\
80155\end{array}$ & $\begin{array}{l}00001 \\
00002 \\
00003 \\
00003 \\
00011 \\
00003 \\
00003\end{array}$ & $\begin{array}{l}07-71 \\
07-71 \\
07-71 \\
10-70 \\
10-74 \\
07-74 \\
07-74\end{array}$ & $\begin{array}{l}09-82 \\
09-82 \\
09-82 \\
09-83 \\
09-83 \\
09-83 \\
09-83\end{array}$ & $\begin{array}{l}3,785 \\
3,775 \\
3,784 \\
4,748 \\
2,655 \\
3,379 \\
3,379\end{array}$ & $\begin{array}{r}295 \\
305 \\
296 \\
0 \\
632 \\
0 \\
0\end{array}$ & $\begin{array}{r}13 \\
13 \\
13 \\
13 \\
9 \\
10 \\
10\end{array}$ & $\begin{array}{r}35.30 \\
24.50 \\
25.00 \\
8,100.00 \\
698.00 \\
343.00 \\
3,540.00\end{array}$ & $\begin{array}{r}-0.29 \\
-0.29 \\
-0.29 \\
.51 \\
130.00 \\
1.00 \\
.02\end{array}$ & $\begin{array}{r}13.47 \\
11.97 \\
12.65 \\
1,215.21 \\
375.08 \\
25.13 \\
132.13\end{array}$ & -- \\
\hline-- & -- & -- & $\begin{array}{l}00060 \\
00060\end{array}$ & $\begin{array}{l}00003 \\
00006\end{array}$ & $\begin{array}{l}08-54 \\
09-75\end{array}$ & $\begin{array}{l}09-83 \\
09-75\end{array}$ & $\begin{array}{r}10,653 \\
8\end{array}$ & $\begin{array}{r}0 \\
22\end{array}$ & $\begin{array}{r}30 \\
1\end{array}$ & $\begin{array}{l}163.00 \\
120.00\end{array}$ & $\begin{array}{r}.00 \\
97.00\end{array}$ & $\begin{array}{r}55.67 \\
106.50\end{array}$ & -- \\
\hline$E$ & 181 & $1970-80$ & - & -- & --- & -.. & - & -- & - & - & -- & - & - \\
\hline E & $18 \quad 19$ & $1970-80$ & -- & -- & --- & --- & -- & -- & -- & -- & - & - & -- \\
\hline$A, B$ & 1 & 1974 & - & -- & -. -- & -- -- & -- & -- & -- & -- & -- & - & -- \\
\hline
\end{tabular}




\begin{tabular}{|c|c|c|c|c|c|c|c|c|}
\hline $\begin{array}{l}\text { Number } \\
\text { on } \\
\text { plate }\end{array}$ & $\begin{array}{l}\text { Station } \\
\text { number }\end{array}$ & Station name & $\begin{array}{l}\text { Lati- } \\
\text { tude }\end{array}$ & $\begin{array}{l}\text { Longi- } \\
\text { tude }\end{array}$ & County & $\begin{array}{l}\text { Hydro- } \\
\text { logic } \\
\text { unit }\end{array}$ & $\begin{array}{l}\text { Drain- } \\
\text { age } \\
\text { area } \\
\text { (square } \\
\text { miles) }\end{array}$ & $\begin{array}{l}\text { Peak-flow } \\
\text { period } \\
\text { of } \\
\text { record }\end{array}$ \\
\hline 63 & 08900400 & $\begin{array}{l}\text { Rio Grande Cochiti to Isleta } \\
\text { X-Section } 4 W, N \text {. Hex. }\end{array}$ & 353454 & 1062116 & Sandoval & 13020201 & -- & -- \\
\hline 64 & 08900402 & $\begin{array}{l}\text { Rio Grande Cochiti to Isleta } \\
\text { X-Section } 4 \mathrm{E} \text {, N. Hex. }\end{array}$ & 353453 & 1062106 & Sandoval & 13020201 & -- & -- \\
\hline 65 & 353427106452710 & $\begin{array}{l}\text { Jemez River at HY4 near San } \\
\text { Isidro, N. Mex. }\end{array}$ & 353427 & 1064527 & Sandoval & 13020202 & -- & -- \\
\hline 66 & 353427106452700 & $\begin{array}{l}\text { Jemez River at HY } 4 \text { near San } \\
\text { Isidro, N. Mex. }\end{array}$ & 353427 & 1064527 & Sandoval & 13020202 & -- & -- \\
\hline 67 & 08900500 & $\begin{array}{l}\text { Rio Grande Cochiti to Isleta } \\
\text { X-Section } 5, N \text {. Mex. }\end{array}$ & 353412 & 1062127 & Sandoval & 13020201 & -- & -- \\
\hline 68 & 353406106513710 & $\begin{array}{l}\text { Arroyo Peñasco at Hwy } 44 \text { San } \\
\text { Isidro, N. Mex. }\end{array}$ & 353406 & 1065137 & Sandoval & 13020202 & -- & -- \\
\hline 69 & 08900600 & $\begin{array}{l}\text { Rio Grande Cochiti to Isleta } \\
\text { X-Section } 6, \mathrm{~N} \text {. Mex. }\end{array}$ & 353331 & 1062139 & Sandoval & 13020201 & -- & -- \\
\hline 70 & 08900700 & $\begin{array}{l}\text { Rio Grande Cochiti to Isleta } \\
\text { X-Section } 7 W, N . \text { Hex. }\end{array}$ & 353300 & 1062142 & Sandoval & 13020201 & -- & -- \\
\hline 71 & 08900701 & $\begin{array}{l}\text { Rio Grande Cochiti to Isleta } \\
\text { X-Section } 7 \mathbb{M}, N \text {. Hex. }\end{array}$ & 353300 & 1062139 & Sandoval & 13020201 & -- & -- \\
\hline 72 & 08900702 & $\begin{array}{l}\text { Rio Grande Cochiti to Isleta } \\
\text { X-Section } 7 E, N \text {. Mex. }\end{array}$ & 353300 & 1062137 & Sandoval & 13020201 & -- & -- \\
\hline 73 & 353240106465610 & $\begin{array}{l}\text { Rio Salado south of San Ysidro, } \\
\text { N. Mex. }\end{array}$ & 353240 & 1064656 & Sandoval & 13020202 & -- & -- \\
\hline 74 & 08900800 & $\begin{array}{l}\text { Rio Grande Cochiti to Isleta } \\
\text { X-Section } 8, N \text {. Mex. }\end{array}$ & 353205 & 1062204 & Sandoval & 13020202 & -- & -- \\
\hline 75 & 08900900 & $\begin{array}{l}\text { Rio Grande Cochiti to Isleta } \\
\text { x-Section } 9 W, N \text {. Mex. }\end{array}$ & 353140 & 1062229 & Sandoval & 13020201 & -- & -- \\
\hline
\end{tabular}




\begin{tabular}{|c|c|c|c|c|c|c|c|c|c|c|c|c|c|}
\hline \multirow{2}{*}{\multicolumn{3}{|c|}{ Water-quality data }} & \multicolumn{11}{|c|}{ Daily-values data } \\
\hline & & & \multirow{2}{*}{$\begin{array}{l}\text { Param- } \\
\text { eter } \\
\text { code }\end{array}$} & \multirow[b]{2}{*}{$\begin{array}{l}\text { Sta- } \\
\text { tistic } \\
\text { code }\end{array}$} & & \multicolumn{3}{|c|}{ Number } & \multirow{2}{*}{ Haximum } & \multirow{2}{*}{ Minimum } & \multirow{2}{*}{ Mean } & \multirow{2}{*}{$\begin{array}{l}\text { Data } \\
\text { source/ } \\
\text { site } \\
\text { name }\end{array}$} \\
\hline $\begin{array}{c}\text { Type } \\
\text { of } \\
\text { analysis }\end{array}$ & $\begin{array}{l}\text { Number } \\
\text { of } \\
\text { analyses }\end{array}$ & $\begin{array}{l}\text { Period } \\
\text { of } \\
\text { secord }\end{array}$ & & & $\frac{\text { reco }}{\text { Begin- }}$ & ord & $\begin{array}{c}\text { Number } \\
\text { of }\end{array}$ & missing & Water & & & & \\
\hline E & 161 & $1970-80$ & -- & -- & -- -- & ---- & -- & -- & -- & -- & -- & -- & -- \\
\hline E & 181 & $1970-80$ & -- & -- & --- & --- & - & -- & -- & - & -- & -- & -- \\
\hline$A, B$ & 21 & $1973-74$ & - & -- & --- & --- & -- & -- & -- & -- & -- & -- & -- \\
\hline$A, B$ & 1 & 1973 & -- & -- & --- & --- & -- & -- & -- & -- & -- & -- & -- \\
\hline E & 161 & $1970-80$ & -- & -- & -- -- & -- -- & -- & -- & -- & -- & -- & -- & -- \\
\hline$A, B$ & 1 & 1974 & -- & - & -- & -- & -- & -- & -- & -- & -- & -- & -- \\
\hline E & 161 & $1970-80$ & -- & -- & --- & $--\cdot$ & -- & -- & -- & -- & -- & -- & -- \\
\hline E & 151 & $1970-80$ & -- & -- & --- & --- & -- & -- & -- & -- & -- & -- & -- \\
\hline E & $\begin{array}{ll}61 \\
6\end{array}$ & $1972-75$ & -- & -- & -- -- & -- & -- & $\cdots$ & -- & -- & -- & -- & -- \\
\hline E & 101 & $1970-75$ & -- & -- & --- & --- & -- & -- & -- & -- & -- & -- & - \\
\hline$A, B$ & 1 & 1974 & -- & -- & ---- & --- & -- & -- & -- & -- & -- & -- & -- \\
\hline$A, E$ & 161 & $1970-80$ & -- & -- & --- & $-\cdots$ & -- & -- & -- & -- & -- & -- & -- \\
\hline$A, E$ & 141 & $1971-80$ & -- & -- & --- & -- -- & -- & -- & -- & -- & -- & -- & - \\
\hline
\end{tabular}


Table 1.--Sumary of surface-vater data-collection sites in the Jenez Mountains, Mer Mexico--Continued

\begin{tabular}{|c|c|c|c|c|c|c|c|c|}
\hline $\begin{array}{c}\text { Number } \\
\text { on } \\
\text { plate } 1\end{array}$ & $\begin{array}{l}\text { Station } \\
\text { number }\end{array}$ & Station name & $\begin{array}{l}\text { Lati- } \\
\text { tude }\end{array}$ & $\begin{array}{l}\text { Longi- } \\
\text { tude }\end{array}$ & County & $\begin{array}{l}\text { Hydro- } \\
\text { logic } \\
\text { unit }\end{array}$ & $\begin{array}{l}\text { Drain- } \\
\text { age } \\
\text { area } \\
\text { (square } \\
\text { miles) }\end{array}$ & $\begin{array}{l}\text { Peak-flow } \\
\text { period } \\
\text { of } \\
\text { record }\end{array}$ \\
\hline 76 & 08900901 & $\begin{array}{l}\text { Rio Grande Cochiti to Isleta } \\
\text { X-Section } 9 \mathrm{E}, \mathrm{N} \text {. Mex. }\end{array}$ & 353138 & 1062226 & Sandoval & 13020201 & -- & -- \\
\hline 77 & 08901001 & $\begin{array}{l}\text { Rio Grande Cochiti to Isleta } \\
\text { X-Section 10E, N. Hex. }\end{array}$ & 353103 & 1062255 & Sandoval & 13020201 & -- & -- \\
\hline 78 & 08901000 & $\begin{array}{l}\text { Rio Grande Cochiti to Isleta } \\
\text { X-Section 10W, N. Mex. }\end{array}$ & 353103 & 1062252 & Sandoval & 13020201 & -- & -- \\
\hline 79 & 08901100 & $\begin{array}{l}\text { Rio Grande Cochiti to Isleta } \\
\text { X-Section } 11, N \text {. Mex. }\end{array}$ & 353029 & 1062352 & Sandoval & 13020201 & -- & -- \\
\hline 80 & 08901200 & $\begin{array}{l}\text { Rio Grande Cochiti to Isleta } \\
\text { X-Section } 12, \mathrm{~N} \text {. Mex. }\end{array}$ & 352951 & 1062348 & Sandoval & 13020201 & -- & -- \\
\hline 81 & 08901300 & $\begin{array}{l}\text { Rio Grande Cochiti to Isleta } \\
\text { X-Section } 13, N \text {. Mex. }\end{array}$ & 352905 & 1062404 & Sandoval & 13020201 & -- & -- \\
\hline 82 & 08901400 & $\begin{array}{l}\text { Rio Grande Cochiti to Isleta } \\
\text { X-Section } 14, N \text {. Mex. }\end{array}$ & 352815 & 1062433 & Sandoval & 13020201 & -- & -- \\
\hline 83 & 08901500 & $\begin{array}{l}\text { Rio Grande Cochiti to Isleta } \\
\text { X-Section } 15, N \text {. Mex. }\end{array}$ & 352738 & 1062506 & Sandoval & 13020201 & -- & -- \\
\hline 84 & 08901600 & $\begin{array}{l}\text { Rio Grande Cochiti to Isleta } \\
\text { X-Section } 16, N \text {. Mex. }\end{array}$ & 352714 & 1062550 & Sandoval & 13020201 & -- & -- \\
\hline 85 & 08319000 & Rio Grande at San Felipe, N. Mex. & 352639 & 1062623 & Sandoval & 13020201 & 16,100 & $1927-83$ \\
\hline 86 & 08901800 & $\begin{array}{l}\text { Rio Grande Cochiti to Isleta } \\
\text { X-Section } 18, N \text {. Mex. }\end{array}$ & 352545 & 1062650 & Sandoval & 13020201 & -- & -- \\
\hline 87 & 08901900 & $\begin{array}{l}\text { Rio Grande Cochiti to Isleta } \\
\text { X-Section } 19, \mathrm{~N} \text {. Mex. }\end{array}$ & 352509 & 1062716 & Sandoval & 13020201 & - & - \\
\hline
\end{tabular}




\begin{tabular}{|c|c|c|c|c|c|c|c|c|c|c|c|c|c|}
\hline \multirow{2}{*}{\multicolumn{3}{|c|}{ Water-quality data }} & \multicolumn{11}{|c|}{ Daily-values data } \\
\hline & & & \multirow[b]{2}{*}{$\begin{array}{l}\text { Param- } \\
\text { eter } \\
\text { code }\end{array}$} & \multirow[b]{2}{*}{$\begin{array}{l}\text { Sta- } \\
\text { tistic } \\
\text { code }\end{array}$} & & \multirow{2}{*}{$\begin{array}{c}\text { Number } \\
\text { of } \\
\text { days }\end{array}$} & \multirow{2}{*}{$\begin{array}{l}\text { Number } \\
\text { of } \\
\text { missing } \\
\text { days }\end{array}$} & \multirow[b]{2}{*}{$\begin{array}{l}\text { Water } \\
\text { years }\end{array}$} & \multirow[b]{2}{*}{ Maximum } & \multirow[b]{2}{*}{ Minimum } & \multirow[b]{2}{*}{ Mean } & \multirow{2}{*}{$\begin{array}{l}\text { Data } \\
\text { source/ } \\
\text { site } \\
\text { name }\end{array}$} \\
\hline $\begin{array}{c}\text { Type } \\
\text { of } \\
\text { analysis }\end{array}$ & $\begin{array}{l}\text { Number } \\
\text { of } \\
\text { analyse }\end{array}$ & $\begin{array}{c}\text { Period } \\
\text { of } \\
\text { es record }\end{array}$ & & & $\begin{array}{c}\text { rec } \\
\text { Begin- } \\
\text { ning }\end{array}$ & Ending & & & & & & & \\
\hline E & 8 & $1972-79$ & -- & -- & --- & --- & -- & -- & -- & -- & -- & -- & -- \\
\hline E & 7 & $1970-75$ & -- & -- & -- -- & --- & -- & -- & -- & -- & -- & -- & -- \\
\hline E & 10 & $1970-75$ & -- & -- & --- & --- & -- & -- & -- & -- & -- & -- & - \\
\hline$E$ & 14 & $1970-80$ & -- & -- & --- & -- -- & -- & -- & -- & -- & -- & -- & -- \\
\hline E & 16 & $1970-80$ & -- & -- & --- & -- -- & -- & -- & -- & -- & -- & -- & -- \\
\hline E & 15 & $1970-82$ & -- & - & -- -- & --- & -- & -- & -- & - & -- & -- & -- \\
\hline E & 18 & $1970-82$ & -- & -- & -- -- & -. -- & -- & -- & -- & -- & -- & -- & -- \\
\hline E & 14 & $1970-80$ & -- & -- & -- -- & -- -- & -- & -- & -- & -- & -- & -- & -- \\
\hline$E$ & 16 & $1970-80$ & -- & -- & --- & -- -- & -- & -- & -- & -- & -- & -- & -- \\
\hline$A, B, C, D, E$ & 133 & $1970-83$ & 00060 & 00003 & $\begin{array}{l}01-27 \\
03-29 \\
05-29 \\
07-29 \\
11-29 \\
10-30\end{array}$ & $\begin{array}{l}12-28 \\
03-29 \\
05-29 \\
08-29 \\
06-30 \\
09-83\end{array}$ & $\begin{array}{r}731 \\
31 \\
31 \\
62 \\
242 \\
19,358\end{array}$ & $\begin{array}{l}0 \\
0 \\
0 \\
0 \\
0 \\
0\end{array}$ & $\begin{array}{r}3 \\
0 \\
0 \\
0 \\
1 \\
53\end{array}$ & $\begin{array}{r}11,460.00 \\
1,636.00 \\
10,520.00 \\
9,560.00 \\
5,310.00 \\
21,300.00\end{array}$ & $\begin{array}{r}131.00 \\
574.00 \\
1,797.00 \\
544.00 \\
360.00 \\
34.00\end{array}$ & $\begin{array}{r}1,926.31 \\
912.16 \\
5,537.93 \\
1,895.72 \\
1,675.55 \\
1,327.88\end{array}$ & -- \\
\hline E & 12 & $1971-80$ & -- & -- & -- & -- -- & -- & -- & -- & -- & -- & -- & -- \\
\hline E & 16 & $1970-80$ & -- & -- & --- & -- -- & -- & -- & - & -- & -- & -- & -- \\
\hline
\end{tabular}


Table 1.--Sumary of surface-water data-collection sites in the Jenez Hountains, Mer Hexico--Continued

\begin{tabular}{|c|c|c|c|c|c|c|c|c|}
\hline $\begin{array}{l}\text { Number } \\
\text { on } \\
\text { plate }\end{array}$ & $\begin{array}{l}\text { Station } \\
\text { number }\end{array}$ & Station name & $\begin{array}{l}\text { Lati- } \\
\text { tude }\end{array}$ & $\begin{array}{l}\text { Longi- } \\
\text { tude }\end{array}$ & County & $\begin{array}{l}\text { Hydro- } \\
\text { logic } \\
\text { unit }\end{array}$ & $\begin{array}{l}\text { Drain- } \\
\text { age } \\
\text { area } \\
\text { (square } \\
\text { miles) }\end{array}$ & $\begin{array}{l}\text { Peak-flow } \\
\text { period } \\
\text { of } \\
\text { record }\end{array}$ \\
\hline 88 & 08902000 & $\begin{array}{l}\text { Rio Grande Cochiti to Isleta } \\
\text { X-Section } 20, \mathrm{~N} \text {. Mex. }\end{array}$ & 352439 & 1062800 & Sandoval & 13020201 & -- & - \\
\hline 89 & 08902101 & $\begin{array}{l}\text { Rio Grande Cochiti to Isleta } \\
\text { X-Section } 21 E, N \text {. Mex. }\end{array}$ & 352349 & 1062829 & Sandoval & 13020201 & - & -- \\
\hline 90 & 08328500 & $\begin{array}{l}\text { Jemez Canyon Reservoir near } \\
\text { Bernalillo, N. Mex. }\end{array}$ & 352340 & 1063245 & Sandoval & 13020202 & 1,030 & -- \\
\hline 91 & 08329000 & $\begin{array}{l}\text { Jemez River below Jemez Canyon } \\
\text { Dam, N. Mex. }\end{array}$ & 352324 & 1063203 & Sandoval & 13020202 & 1,030 & $1936-83$ \\
\hline 92 & 08902200 & $\begin{array}{l}\text { Rio Grande Cochiti to Isleta } \\
\text { X-Section } 22, N \text {. Hex. }\end{array}$ & 352316 & 1062858 & Sandoval & 13020201 & -- & -- \\
\hline 93 & 08902301 & $\begin{array}{l}\text { Rio Grande Cochiti to Isleta } \\
\text { X-Section 23E, N. Hex. }\end{array}$ & 352249 & 1062954 & Sandoval & 13020201 & - & -- \\
\hline 94 & 08902300 & $\begin{array}{l}\text { Rio Grande Cochiti to Isleta } \\
\text { X-Section 23W, N. Mex. }\end{array}$ & 352249 & 1062951 & Sandoval & 13020201 & - & -- \\
\hline 95 & 352247106295400 & $\begin{array}{l}\text { Rio Grande at Angustura Hdg., } \\
\text { N. Mex. }\end{array}$ & 352247 & 1062954 & Sandoval & 13020201 & -- & -- \\
\hline 96 & 352246106295600 & $\begin{array}{l}\text { Angustura Wasteway below Drain, } \\
\text { N. Mex. }\end{array}$ & 352246 & 1062956 & Sandoval & 13020201 & -- & -- \\
\hline 97 & 08902401 & $\begin{array}{l}\text { Rio Grande Cochiti to Isleta } \\
\text { X-Section } 24 \mathrm{E}, \mathrm{N} \text {. Hex. }\end{array}$ & 352227 & 1063108 & Sandoval & 13020201 & -- & -- \\
\hline
\end{tabular}




\begin{tabular}{|c|c|c|c|c|c|c|c|c|c|c|c|c|c|}
\hline \multirow{2}{*}{\multicolumn{3}{|c|}{ Water-quality data }} & \multicolumn{11}{|c|}{ Daily-values data } \\
\hline & & & \multirow[b]{2}{*}{$\begin{array}{c}\text { Param- } \\
\text { eter } \\
\text { code }\end{array}$} & \multirow[b]{2}{*}{$\begin{array}{l}\text { Sta- } \\
\text { tistic } \\
\text { code }\end{array}$} & & \multirow{2}{*}{$\begin{array}{c}\text { Number } \\
\text { of } \\
\text { days }\end{array}$} & & \multirow{2}{*}{ Water } & \multirow[b]{2}{*}{ Maximum } & \multirow[b]{2}{*}{ Minimum } & \multirow[b]{2}{*}{ Mean } & \multirow{2}{*}{$\begin{array}{l}\text { Data } \\
\text { source/ } \\
\text { site } \\
\text { name }\end{array}$} \\
\hline $\begin{array}{c}\text { Type } \\
\text { of } \\
\text { analysis }\end{array}$ & $\begin{array}{l}\text { Number } \\
\text { of } \\
\text { analyses }\end{array}$ & $\begin{array}{l}\text { Period } \\
\text { of } \\
\text { record }\end{array}$ & & & $\begin{array}{c}\text { rec } \\
\text { Begin- } \\
\text { ning }\end{array}$ & Ending & & $\begin{array}{l}x \text { of } \\
\text { missino } \\
\text { days }\end{array}$ & & & & & \\
\hline E & 15 & $1971-80$ & - & -- & -. - & -.. & -- & -- & -- & -- & - & -- & - \\
\hline E & 15 & $1970-80$ & - & - & -. & -.. & -- & -- & -- & - & -. & - & - \\
\hline-- & - & -- & $\begin{array}{l}00054 \\
00054\end{array}$ & $\begin{array}{l}30800 \\
32400\end{array}$ & $\begin{array}{l}10-69 \\
10-65 \\
01-77\end{array}$ & $\begin{array}{l}12-76 \\
09-69 \\
09-83\end{array}$ & $\begin{array}{l}2,649 \\
1,461 \\
2,464\end{array}$ & $\begin{array}{l}0 \\
0 \\
0\end{array}$ & $\begin{array}{l}8 \\
4 \\
7\end{array}$ & $\begin{array}{r}25,750.00 \\
2,800.00 \\
20,250.00\end{array}$ & $\begin{array}{r}0.00 \\
.00 \\
.00\end{array}$ & $\begin{array}{r}276.25 \\
134.79 \\
1,807.64\end{array}$ & - \\
\hline$A, B, C$ & 2931 & $1966-83$ & $\begin{array}{l}80154 \\
80155\end{array}$ & $\begin{array}{l}00003 \\
00003\end{array}$ & $\begin{array}{l}04-36 \\
04-43 \\
10-69 \\
02-70 \\
08-70 \\
10-70 \\
04-71 \\
09-71 \\
10-55 \\
10-61 \\
10-55\end{array}$ & $\begin{array}{l}09-37 \\
09-83 \\
11-69 \\
06-70 \\
08-70 \\
10-70 \\
05-71 \\
09-71 \\
09-58 \\
09-62 \\
09-58\end{array}$ & $\begin{array}{r}548 \\
14,793 \\
14 \\
47 \\
2 \\
7 \\
14 \\
1 \\
991 \\
327 \\
1,052\end{array}$ & $\begin{array}{r}0 \\
0 \\
47 \\
103 \\
29 \\
24 \\
47 \\
29 \\
105 \\
38 \\
44\end{array}$ & $\begin{array}{r}2 \\
41 \\
1 \\
0 \\
0 \\
1 \\
0 \\
0 \\
3 \\
1 \\
3\end{array}$ & $\begin{array}{r}1,480.00 \\
3,640.00 \\
1,670.00 \\
1,720.00 \\
3,030.00 \\
1,120.00 \\
943.00 \\
758.00 \\
101,000.00 \\
39,400.00 \\
94,400.00\end{array}$ & $\begin{array}{r}.00 \\
.00 \\
833.00 \\
385.00 \\
1,450.00 \\
746.00 \\
735.00 \\
758.00 \\
.00 \\
130.00 \\
.00\end{array}$ & $\begin{array}{r}120.10 \\
56.25 \\
1,081.50 \\
839.89 \\
2,240.00 \\
866.42 \\
786.50 \\
758.00 \\
4,213.21 \\
2,997.36 \\
1,166.16\end{array}$ & -- \\
\hline $\mathrm{E}$ & 141 & $1971-80$ & $\cdots$ & -- & $-\cdots$ & -. & - & - & -- & -- & - & -- & -- \\
\hline $\mathrm{E}$ & 101 & $1971-75$ & - & - & -- & -- & -- & - & - & - & - & - & - \\
\hline$E$ & 131 & $1970-82$ & - & - & $\cdots$ & - . & - & -- & - & - & - & - & -. \\
\hline$A, B$ & 1 & 1970 & -- & -- & -- & -. - & -- & -- & - & -- & -- & - & - \\
\hline$A, B$ & 1 & 1970 & - & -- & -- & -. - & -- & - & -- & - & - & - & - \\
\hline E & 81 & $1972-75$ & $\cdots$ & - & -- -. & -.. & -- & -- & -- & - & - & - & - \\
\hline
\end{tabular}


Table 1.--Sumary of surface-water data-collection sites in the Jenez Mountains, Mev Mexico--Concluded

\begin{tabular}{|c|c|c|c|c|c|c|c|c|}
\hline $\begin{array}{l}\text { Number } \\
\text { on } \\
\text { plate } 1\end{array}$ & $\begin{array}{l}\text { Station } \\
\text { number }\end{array}$ & Station name & $\begin{array}{l}\text { Lati- } \\
\text { tude }\end{array}$ & $\begin{array}{l}\text { Longi- } \\
\text { tude }\end{array}$ & County & $\begin{array}{l}\text { Hydro- } \\
\text { logic } \\
\text { unit }\end{array}$ & $\begin{array}{l}\text { Drain- } \\
\text { age } \\
\text { area } \\
\text { (square } \\
\text { miles) }\end{array}$ & $\begin{array}{l}\text { Peak-flow } \\
\text { period } \\
\text { of } \\
\text { record }\end{array}$ \\
\hline 98 & 08902400 & $\begin{array}{l}\text { Rio Grande Cochiti to Isleta } \\
\text { X-Section } 24 \mathrm{~W}, \mathrm{~N} \text {. Mex. }\end{array}$ & 352227 & 1063106 & Sandoval & 13020201 & -- & -- \\
\hline 99 & 08902501 & $\begin{array}{l}\text { Rio Grande Cochiti to Isleta } \\
\text { X-Section } 25 \mathrm{E}, \mathrm{N} \text {. Mex. }\end{array}$ & 352151 & 1063100 & Sandoval & 13020201 & - & -- \\
\hline 100 & 08902500 & $\begin{array}{l}\text { Rio Grande Cochiti to Isleta } \\
\text { X-Section } 25 \mathrm{~W}, \mathrm{~N} \text {. Mex. }\end{array}$ & 352151 & 1063058 & Sandoval & 13020203 & -- & -- \\
\hline
\end{tabular}




\begin{tabular}{|c|c|c|c|c|c|c|c|c|c|c|c|c|c|}
\hline \multirow{2}{*}{\multicolumn{3}{|c|}{ Water-quality data }} & \multicolumn{11}{|c|}{ Daily-values data } \\
\hline & & & \multirow[b]{2}{*}{$\begin{array}{l}\text { Param- } \\
\text { eter } \\
\text { code }\end{array}$} & \multirow[b]{2}{*}{$\begin{array}{l}\text { Sta- } \\
\text { tistic } \\
\text { code }\end{array}$} & & \multirow{2}{*}{$\begin{array}{c}\text { Number } \\
\text { of } \\
\text { days }\end{array}$} & Number & \multirow[b]{2}{*}{ Water } & \multirow[b]{2}{*}{ Maximum } & \multirow[b]{2}{*}{ Minimum } & \multirow[b]{2}{*}{ Kean } & \multirow{2}{*}{$\begin{array}{l}\text { Data } \\
\text { source, } \\
\text { site } \\
\text { name }\end{array}$} \\
\hline $\begin{array}{c}\text { Type } \\
\text { of } \\
\text { analysis }\end{array}$ & $\begin{array}{l}\text { Number } \\
\text { of } \\
\text { analyses }\end{array}$ & $\begin{array}{l}\text { Period } \\
\text { of } \\
\text { s record }\end{array}$ & & & $\begin{array}{l}\text { reco } \\
\text { Begin- } \\
\text { ning }\end{array}$ & $\frac{\text { ord }}{\text { Ending }}$ & & $\begin{array}{l}\text { of } \\
\text { missing } \\
\text { days }\end{array}$ & & & & & \\
\hline$A, E$ & 131 & $1971-82$ & -- & -- & ---- & --- & -- & -- & -- & -- & -- & -- & -- \\
\hline$A, E$ & 101 & $1971-79$ & - & -- & ---- & --- & -- & -- & -- & -- & -- & -- & -- \\
\hline$A, E$ & 151 & $1970-82$ & -- & -- & --- & --- & -- & -- & -- & -- & -- & -- & -- \\
\hline
\end{tabular}


Table 2.--Summary of ground-water data-collection

sites in the Jemez Mountains. New Mexico

$[-$, no data $]$

EXPLANATION

Type: GW, well; SP, spring.

Principal aquifer: 000EXRV-unknown, extrusive rocks; 110AVMB-Cenozoic, Quaternary alluvium, bolson deposits, and other surface deposits; 110PTODCenozoic, Quaternary pediment, terrace, and other deposits of gravel, sand, and caliche; 112BDLR-Cenozoic, Quaternary, Pleistocene Bandelier Rhyolite Tuff of Tewa Group; 112BLSP-Cenozoic, Quaternary, Pleistocene Battleship Rock Flow; 112SNTF-Cenozoic, Quaternary, Pleistocene Santa Fe Group; 112TSRG-Cenozoic, Quaternary, Pleistocene Tshirege Member of Bandelier Tuff of Tewa Group; 112VLLS-Cenozoic, Quaternary, Pleistocene Valles Rhyolite of Tewa Group; 121PUYEF-Cenozoic, Tertiary, Pliocene Puye Conglomerate, Fanglomerate Member; 121TCCM-Cenozoic, Tertiary, P1iocene Tschicoma Formation; 121TSUQ-Cenozoic, Tertiary, Pliocene Tesuque Formation, undifferentiated unit; 122SNTFL-Cenozoic, Tertiary, Miocene Santa Fe Group, lower part; 210MNCS-Mesozoic, Upper Cretaceous Mancos Shale; 211DKOTMesozoic, Upper Cretaceous Dakota Sandstone or Formation; 211MENF-Mesozoic, Upper Cretaceous Menefee Formation; 2110JAM-Mesozoic, Upper Cretaceous 0jo Alamo Sandstone; 221AGZC-Mesozoic, Upper Jurassic Agua Zarca Sandstone Member of Sierra Nacimiento; 221MRSN-Mesozoic, Upper Jurassic Morrison Formation; 231CHNL-Mesozoic, Upper Triassic Chinle Formation; 231SRMPMesozoic, Upper Triassic Shinarump Member of Chinle Formation; 318ABO L-Paleozoic, Lower Permian, Leonardian Abo Sandstone (Lower Tongue); 325MDER-Paleozoic, Middle Pennsylvanian, Des Moinesian Madera Limestone; 326MGDL-Paleozoic, Middle Pennsylvanian, Atokan Magdalena Group; 400PCMBPrecambrian Erathem.

Data available: A, major- and minor-ion analysis; B, trace-element analysis; $C$, radiochemical analysis; $D$, water-level data.

Water use: H, domestic; $\mathrm{P}$, public supply; S, stock; U, unused.

Source of data and additional data sources: QW, WATSTORE water-quality file; GWSI, WATSTORE Ground-Water Site-Inventory file; HD, WATSTORE header file; T1978, Trainer, 1978; G1982, Goff and others, 1982; J1967, John and others, 1967; FHS, Fenton Hil1 Series published by Los Alamos Nationa1 Laboratories (year specifies in which year the data were collected); DOF, data on file at U.S. Geological Survey, Water Resources Division, New Mexico District office (year specifies in which year the data were collected). Numbers or names in parentheses identify the well name the author used if other than the U.S. Geological Survey name. References for the other available data are listed in the References Cited section of this report. 


\begin{tabular}{|c|c|c|c|c|c|c|}
\hline $\begin{array}{l}\text { Number } \\
\text { on } \\
\text { plate } 2\end{array}$ & $\begin{array}{l}\text { Lati- } \\
\text { tude }\end{array}$ & $\begin{array}{l}\text { Longi- } \\
\text { tude }\end{array}$ & Local identifier & Type & owner & $\begin{array}{l}\text { Principal } \\
\text { aquifer }\end{array}$ \\
\hline 101 & $35 \quad 22 \quad 25$ & 1062938 & $13 \mathrm{~N} .04 \mathrm{E} .11 .113$ & GW & Stones, T.P. & - \\
\hline 102 & $35 \quad 23 \quad 36$ & 1063255 & $14 \mathrm{~N} .04 \mathrm{E} .31 .444$ & GW & -- & 110AVIB \\
\hline 103 & $35 \quad 2533$ & 1063658 & 14N.03E.22.323 & GW & San Ana Pueblo & $110 \mathrm{AVMB}$ \\
\hline 104 & 352540 & 1063659 & 14N.03E.22.311 & GW & -- & - \\
\hline 105 & 352540 & 1063659 & $14 \mathrm{~N} .03 \mathrm{E} .22 .311$ & GW & -- & $\cdots$ \\
\hline 106 & 352540 & 1063659 & 14N.03E.22.323 & GW & - & -- \\
\hline 107 & $35 \quad 2607$ & 1062643 & 14 N.05E.19.221 & GW & San Felipe & 112 SNTF \\
\hline 108 & $35 \quad 2614$ & 1063954 & 14N.03E.18.340 & GW & $-\infty$ & $112 \mathrm{SNTF}$ \\
\hline 109 & $35 \quad 2757$ & 1063623 & $14 \mathrm{~N} .03 \mathrm{E} .03 .434$ & GW & $\mathrm{BIA}$ & 112SNTF \\
\hline 110 & $35 \quad 2810$ & 1064522 & $14 \mathrm{~N} .02 \mathrm{E} .05 .320$ & GW & -- & $112 \mathrm{SNTF}$ \\
\hline 111 & $\begin{array}{lll}35 & 28 & 13\end{array}$ & 1063931 & 14N.03E.06.423 & GW & BLM & -- \\
\hline 112 & $35 \quad 2904$ & $10641 \quad 14$ & 15N.02E.36.314 & GW & Galvan, Jose C. & $110 \mathrm{AVIB}$ \\
\hline 113 & $3530 \quad 16$ & 1064316 & 15N.02E. 27.112 & GW & Zia Pueblo & -- \\
\hline 114 & $3530 \quad 16$ & 1064316 & 15N.02E.27.100 & GW & - & $\cdots$ \\
\hline 115 & 353030 & 1064745 & 15N 01E 26222 & $\mathrm{GW}$ & -- & - \\
\hline 116 & $35 \quad 30 \quad 30$ & 1064746 & $15 \mathrm{~N} .01 \mathrm{E} .26 .222$ & GW & $\mathrm{BIA}$ & 110AVKB \\
\hline 117 & $35 \quad 30 \quad 42$ & $106 \quad 42 \quad 44$ & $15 \mathrm{~N} .02 \mathrm{E} .22 .400$ & GW & Zia Pueblo & 112 SNTF \\
\hline 118 & $35 \quad 30 \quad 43$ & 1064318 & 15N.02E.22.300 & GW & - & - \\
\hline 119 & $35 \quad 30 \quad 44$ & 1064251 & 15N.02E. 22.414 & GW & -- & 112SNTF \\
\hline 120 & 353045 & 1064241 & $15 N .02 E .22 .423$ & GW & -- & 112SNTF \\
\hline 121 & $35 \quad 3045$ & $106 \quad 4252$ & 15N.02E.22.330 & GW & - & 110AVIB \\
\hline 122 & 353058 & 1065046 & 15N.01E.21.134 & SP & $\cdots$ & $231 \mathrm{CHNL}$ \\
\hline 123 & $\begin{array}{lll}35 & 31 & 04\end{array}$ & 1065036 & 15N.01E.21.141 & SP & -- & 231CENL \\
\hline 124 & $\begin{array}{lll}35 & 31 & 29\end{array}$ & $106 \quad 22 \quad 12$ & 15N.05E.13.330 & GW & St Dom Pueblo & 112 SNTF \\
\hline 125 & 353135 & 1065045 & $15 \mathrm{~N} .01 \mathrm{E} .16 .313$ & $\mathrm{SP}$ & $\cdots$ & - \\
\hline 126 & 353146 & 1064644 & 15N.01E.13.422 & $\mathrm{GW}$ & Henningsen & $110 \mathrm{AVMB}$ \\
\hline 127 & $35 \quad 3152$ & 1065049 & 15N.01E.16.233 & $\mathrm{SP}$ & - & 231CENL \\
\hline 128 & $\begin{array}{lll}35 & 32 & 13\end{array}$ & 1065049 & 15N.01E.16.111 & $\mathrm{SP}$ & - & - \\
\hline 129 & $\begin{array}{lll}35 & 32 & 26\end{array}$ & $10640 \quad 45$ & 15N.02E.12.432 & $\mathrm{GW}$ & Zia Pueblo & $110 \mathrm{AVMB}$ \\
\hline 130 & $3532 \quad 26$ & 1064045 & $15 \mathrm{~N} .02 \mathrm{E} .12 .431$ & GW & -. & $112 \mathrm{SNTF}$ \\
\hline
\end{tabular}




$\begin{aligned} & \text { Altitude } \\ & \text { of land- }\end{aligned}$ Total
surface depth
datum of Casing Depth Data Number Period
(feet above well diameter cased avail- of of Water $\begin{gathered}\text { Source of data and additional data sources } \\ \text { (site name if other than WATSTORE name) }\end{gathered}$
sea level) (feet) (inches) (feet) able analyses record use

\begin{tabular}{|c|c|c|c|c|c|c|c|c|}
\hline 5,165 & 40 & -- & -- & -- & -- & -- & H & GWSI. \\
\hline- & -- & -- & -- & $A, B$ & 1 & 1950 & - & QW. \\
\hline 5,237 & 29 & -- & -- & $A, B$ & 1 & 1924 & $S$ & QW, GWSI. \\
\hline-- & -- & -- & -- & A & 1 & 1954 & -- & QW. \\
\hline- & -- & -- & -- & $A$ & 1 & 1954 & -- & QW. \\
\hline-- & -- & -- & -- & -- & -- & -- & - & HD. \\
\hline 5,140 & 98 & 10.00 & 98 & $A, B$ & 1 & 1965 & $\mathrm{P}$ & QN, GWSI. \\
\hline-- & -- & -- & -- & -- & -- & -- & -- & HD. \\
\hline 5,725 & 637 & 6.62 & 637 & A & 1 & 1959 & $S$ & $Q W$, GWSI. \\
\hline-- & -- & -- & -- & -- & -- & -- & -- & $\mathrm{HD}$. \\
\hline 5,320 & 43 & 6.00 & 43 & -- & - & -- & $S$ & GWSI. \\
\hline 5,344 & 18 & -- & -- & A & 1 & 1951 & $\mathrm{H}, \mathrm{S}$ & QW, GWSI. \\
\hline 5,410 & 8 & -- & -- & -- & -- & -- & $\mathrm{H}, \mathrm{P}$ & GWSI. \\
\hline- & -- & -- & -- & A & 1 & 1952 & -- & QW. \\
\hline-- & -- & -- & -- & $\mathrm{A}, \mathrm{B}$ & 1 & 1975 & -- & QW. \\
\hline 5,515 & 117 & -- & -- & A & 1 & 1959 & $S$ & QW, GWSI. \\
\hline 5,500 & 335 & 8.62 & 335 & $\AA, B$ & 1 & 1965 & $P$ & QN, GWSI. \\
\hline-- & -- & -- & -- & A & 1 & 1952 & -- & QW. \\
\hline-- & -- & -- & -- & A & 1 & 1974 & -- & QW. \\
\hline-- & -- & -- & -- & $\AA$ & 1 & 1960 & -- & QW. \\
\hline-- & -- & -- & -- & A & 1 & 1951 & -- & QW. \\
\hline-- & -- & -- & - & $\mathrm{A}, \mathrm{B}$ & 1 & 1975 & -- & QW. \\
\hline-- & -- & - & -- & $\mathrm{A}, \mathrm{B}$ & 1 & 1975 & -- & QW. \\
\hline 5,185 & 82 & 10.75 & 82 & $\mathrm{~A}, \mathrm{~B}$ & 1 & 1965 & $\mathrm{P}$ & QW, GWSI. \\
\hline-- & -- & -- & -- & $A, B$ & 1 & 1974 & -- & QW, T1978 (A5). \\
\hline 5,345 & 12 & -- & -- & $A, B$ & 1 & 1959 & $\mathrm{~S}$ & QW, GWSI. \\
\hline-- & -- & -- & -- & $\mathrm{A}, \mathrm{B}$ & 1 & 1974 & -- & QW, T1978 (A4). \\
\hline-- & -- & -- & -- & $A, B$ & 1 & 1974 & -- & QW, T1978 (A3). \\
\hline 5,750 & 503 & 1.00 & 503 & - & -- & -- & $S$ & GWSI. \\
\hline-- & -- & -- & -- & $\mathrm{A}, \mathrm{B}$ & 1 & 1974 & -- & QW. \\
\hline
\end{tabular}




\begin{tabular}{|c|c|c|c|c|c|c|}
\hline $\begin{array}{l}\text { Number } \\
\text { on } \\
\text { plate } 2\end{array}$ & $\begin{array}{l}\text { Lati- } \\
\text { tude }\end{array}$ & $\begin{array}{l}\text { Longi- } \\
\text { tude }\end{array}$ & Local identifier & Type & owner & $\begin{array}{c}\text { Principal } \\
\text { aquifer }\end{array}$ \\
\hline 131 & 353234 & 1065008 & 15N.01E.09.414 & SP & -- & 231CHNL \\
\hline 132 & 353236 & 1064544 & 15N.02E.07.422 & GW & -- & -- \\
\hline 133 & 353238 & 1063429 & 15N.03E.12.322 & GW & BIA & $112 \mathrm{SNTF}$ \\
\hline 134 & 353238 & 1064949 & 15N.01E.10.311 & SP & - & 110AVKB \\
\hline 135 & 353242 & 1064907 & 15N.01W.10.1411 Salt Mound Spring & $\mathrm{SP}$ & -- & $231 \mathrm{CHNL}$ \\
\hline 136 & 353249 & 1064933 & 15N.01E.10.141 & SP & -- & 231CHNL \\
\hline 137 & 353333 & $106 \quad 4614$ & 15N.02E.06.000 & $G W$ & - & 110AVMB \\
\hline 138 & 353355 & 1064614 & $15 \mathrm{~N} .02 \mathrm{E} .06 .22$ & GW & -- & -- \\
\hline 139 & 353400 & 1064850 & 16N.01E.34.44 Rio Salado Well BLM & GW & -- & 231CHNL \\
\hline 140 & 353403 & 1062016 & $16 \mathrm{~N} .06 \mathrm{E} .31 .444$ & GW & Sandoval, B. & $110 \mathrm{AVKB}$ \\
\hline 141 & 353454 & 1063847 & 16N.03E.29.344 0jo Chamisa Sp & SP & -- & 122SNTFL \\
\hline 142 & 353455 & 1063042 & $16 \mathrm{~N} .04 \mathrm{E} .27 .333$ & GW & - & $112 \mathrm{SNTF}$ \\
\hline 143 & 353510 & 1064635 & 16 N.02E. 30.323 & SP & -- & 110AVMB \\
\hline 144 & $35 \quad 3517$ & 1064636 & 16N.01E.25.244 Bluewater Sp & $\mathrm{SP}$ & -- & 231CHNL \\
\hline 145 & 353528 & 1064511 & 16N.02E.29.142 & $\mathrm{SP}$ & -- & -- \\
\hline 146 & 353528 & 1065736 & 16N.01W.29.232 0jito Sp & SP & -- & 210RNCS \\
\hline 147 & 353529 & 1064507 & $16 \mathrm{~N} .02 \mathrm{E} .29 .142$ & SP & -- & $110 \mathrm{AVMB}$ \\
\hline 148 & 353533 & 1064253 & $16 \mathrm{~N} .02 \mathrm{E} .27 .213$ & GW & -- & $112 \operatorname{SNTF}$ \\
\hline 149 & 353552 & 1064249 & 16N.02E. 10.000 & $\mathrm{SP}$ & -- & 231CHNL \\
\hline 150 & 353552 & 1064533 & 16N.02E.20.332 Salt Sp & $\mathrm{SP}$ & -- & $231 \mathrm{CHNL}$ \\
\hline 151 & 353606 & 1065120 & $16 \mathrm{~N} .01 \mathrm{E} .20 .411$ & $\mathrm{SP}$ & - & 325MDER \\
\hline 152 & 353623 & 1065248 & 16N.01E.19.100 & GW & BIA & -- \\
\hline 153 & 353625 & 1065252 & $16 \mathrm{~N} .01 W .19 .1$ & $\mathrm{SP}$ & -- & $110 \mathrm{AVMB}$ \\
\hline 154 & 353638 & 1062107 & $16 \mathrm{~N} .06 \mathrm{E} .18 .300$ & GW & Cochiti Pu & $110 \mathrm{AVKB}$ \\
\hline 155 & 353700 & 1064357 & 16N.02E.16.411 & $\mathrm{GW}$ & - & $110 \mathrm{AVMB}$ \\
\hline 156 & 353707 & 1063329 & 09N.04E.06.411 & GW & US Army & - \\
\hline 157 & 353743 & 1064548 & $16 \mathrm{~N} .02 \mathrm{E} .07 .423$ & $\mathrm{SP}$ & - & 325MDER \\
\hline 158 & 353744 & $10642 \quad 29$ & $16 \mathrm{~N} .02 \mathrm{E} \cdot 10.424$ & SP & -- & 231CHNL \\
\hline 159 & 353750 & 1064554 & 16N.02E.18.214 Tunnel Sp & $\mathrm{SP}$ & -- & $318 \mathrm{ABO} \mathrm{L}$ \\
\hline 160 & 353757 & 1064139 & 16N.02E.11.234 & SP & -- & 231CHNL \\
\hline
\end{tabular}




\begin{tabular}{|c|c|c|c|c|c|c|c|c|}
\hline $\begin{array}{l}\text { Altitude } \\
\text { of land- } \\
\text { surface } \\
\text { datum } \\
\text { (feet above } \\
\text { sea level) }\end{array}$ & $\begin{array}{c}\text { Total } \\
\text { depth } \\
\text { of } \\
\text { well } \\
\text { (feet) }\end{array}$ & $\begin{array}{l}\text { Casing } \\
\text { diameter } \\
\text { (inches) }\end{array}$ & $\begin{array}{l}\text { Depth } \\
\text { cased } \\
\text { (feet) }\end{array}$ & $\begin{array}{c}\text { Data } \\
\text { avail- } \\
\text { able }\end{array}$ & $\begin{array}{c}\text { Number } \\
\text { of } \\
\text { analyses }\end{array}$ & $\begin{array}{l}\text { Period } \\
\text { of } \\
\text { s record }\end{array}$ & $\begin{array}{c}\text { Water } \\
\text { use }\end{array}$ & $\begin{array}{l}\text { Source of data and additional data sources } \\
\text { (site name if other than WATSTORE name) }\end{array}$ \\
\hline-- & -- & - & - & $A, B$ & 3 & $1924-75$ & -- & QW, T1978 (A1). \\
\hline-- & -- & -- & -- & $A$ & 1 & 1954 & -- & QW. \\
\hline 6,240 & 1,105 & 6.62 & 1,105 & $\AA$ & 1 & 1960 & $S$ & QW, GWSI. \\
\hline-- & -- & -- & - & $A, B, C$ & 4 & $1973-74$ & -- & QW. \\
\hline 5,495 & - & - & - & $A, B$ & 1 & 1982 & -- & QW. \\
\hline-- & -- & - & - & $A, B$ & 2 & $1924-75$ & -- & QW. \\
\hline-- & -- & -- & -- & $A, B$ & 1 & 1924 & -- & QW. \\
\hline-- & -- & -- & - & $A$ & 2 & 1954 & -- & QW. \\
\hline-- & 200 & -- & - & -- & - & -- & -- & GWSI. \\
\hline 5,230 & 80 & 8.00 & 80 & $A, B$ & 1 & 1965 & H & QW, GWSI. \\
\hline-- & -- & -- & -- & $\mathrm{A}, \mathrm{B}$ & 1 & 1973 & -- & QW, T1978 (B7). \\
\hline-- & - & - & -- & $A$ & 1 & 1959 & -- & QW. \\
\hline-- & -- & -- & -- & $A, B$ & 2 & 1973 & - & QW，T1978 (A12). \\
\hline-- & -- & -- & - & $A, B$ & 1 & 1973 & -- & QW，T1978 (A7). \\
\hline-- & -- & - & - & $\mathrm{A}, \mathrm{B}$ & 1 & 1962 & - & QW. \\
\hline-- & -- & -- & -- & $\mathrm{A}, \mathrm{B}$ & 1 & 1973 & -- & QW，T1978 (C4). \\
\hline-- & -- & -- & -- & $A, B, C$ & 1 & 1973 & -- & QW, T1978 (A11). \\
\hline- & -- & -- & -- & $A, B$ & 1 & 1974 & -- & QW. \\
\hline-- & -- & - & -- & - & -- & -- & -- & BD. \\
\hline-- & -- & -- & -- & $A, B$ & 1 & 1973 & -- & QW, T1978 (A10), G1982 (VA-36). \\
\hline-- & -- & -- & -- & A & 1 & 1924 & -- & QW. \\
\hline-- & 50 & -- & - & $\AA$ & 1 & 1959 & -- & QW, GWSI. \\
\hline-- & -- & -- & - & $\mathrm{A}, \mathrm{B}$ & 1 & 1924 & -- & QW. \\
\hline 5,260 & 88 & 6.62 & 88 & $A, B$ & 1 & 1965 & $\mathrm{p}$ & QW, GWSI. \\
\hline- & - & -- & -- & $A, B$ & 3 & $1962-73$ & -- & QW， T1978 (E4). \\
\hline 5,362 & 1,000 & 14.00 & 1,000 & -- & -- & -- & $\mathrm{p}$ & GWSI. \\
\hline- & - & -- & - & $A, B$ & 3 & $1953-74$ & -- & QW, T1978 (A8), G1982 (VA-38 Onl Spring). \\
\hline-- & -- & -- & - & $A, B$ & 3 & $1958-73$ & -- & QW, T1978 (E1), G1982 (VA-39 Unnamed Spring). \\
\hline-- & -- & -- & -- & $A, B$ & 1 & 1973 & -- & QW, T1978 (A9). \\
\hline-- & -- & -- & -- & $A, B$ & 1 & 1973 & -- & QW, T1978 (E2). \\
\hline
\end{tabular}




\begin{tabular}{|c|c|c|c|c|c|c|}
\hline $\begin{array}{l}\text { Number } \\
\text { on } \\
\text { plate } 2\end{array}$ & $\begin{array}{l}\text { Lati- } \\
\text { tude }\end{array}$ & $\begin{array}{l}\text { Longi- } \\
\text { tude }\end{array}$ & Local identifier & Type & Owner & $\begin{array}{l}\text { Principal } \\
\text { aquifer }\end{array}$ \\
\hline 161 & $35 \quad 38 \quad 32$ & 1064904 & $16 \mathrm{~N} .01 \mathrm{E} .03 .441$ & $\mathrm{SP}$ & -- & 318ABO L \\
\hline 162 & 353838 & 1065327 & $16 \mathrm{~N} .01 W .01 .410$ & GW & -- & 231SRMP \\
\hline 163 & 353838 & 1065327 & $16 \mathrm{~N} .01 W .01 .410$ & GW & -- & $221 A G 2 C$ \\
\hline 164 & $3538 \quad 39$ & 1065328 & $16 \mathrm{~N} .01 W .01 .4211$ & GW & -- & $326 \mathrm{HGDL}$ \\
\hline 165 & 353843 & 1065318 & $16 \mathrm{~N} .01 \% .01 .410^{\circ}$ & GW & -- & -- \\
\hline 166 & 353843 & 1065318 & $16 \mathrm{~N} .01 W .01 .410$ & GW & -- & -- \\
\hline 167 & $3538 \quad 44$ & 1065319 & $16 \mathrm{~N} .01$ W.01.4211 & SP & -- & 326MGDL \\
\hline 168 & 353849 & 1065057 & 16N.01E.05.244 Log Sp & $\mathrm{SP}$ & -- & 400PCXB \\
\hline 169 & 353906 & 1065215 & 0jo del Espiritu Santo Grant & SP & -- & 231CENL \\
\hline 170 & 353913 & 1064417 & $17 \mathrm{~N} .02 \mathrm{E} .33 .300$ & GW & -- & 110AVIB \\
\hline 171 & 353916 & 1064047 & Cañon de San Diego Grant & $\mathrm{SP}$ & -- & 231CHNL \\
\hline 172 & 353938 & 1065313 & Zia Indian Reservation Cuehana Sp & $\mathrm{SP}$ & -- & 110PTOD \\
\hline 173 & 354019 & 1064530 & $17 \mathrm{~N} .02 \mathrm{E} \cdot 29.311$ & GW & -- & -- \\
\hline 174 & 354030 & 1063257 & $17 N .04 E .29 .133$ & SP & -- & OOOEXRV \\
\hline 175 & 354043 & 1063508 & Cañada de Cochiti Grant & SP & -- & OOOEXRV \\
\hline 176 & 354113 & 1064410 & $17 \mathrm{~N} .02 \mathrm{E} .21 .144$ & GW & -- & $318 \mathrm{ABO} \mathrm{L}$ \\
\hline 177 & $3541 \quad 13$ & $10644 \quad 14$ & $17 N .02 E .21 .144$ & GW & -- & $110 \mathrm{AVNB}$ \\
\hline 178 & 354140 & 1064757 & Cañon de San Diego Grant Crow Sp & SP & - & $318 \mathrm{ABO} \mathrm{L}$ \\
\hline 179 & 354209 & 1064718 & Cañon de San Diego Grant & $\mathrm{SP}$ & -- & 318ABO L \\
\hline 180 & $3542 \quad 13$ & 1063726 & 17N.03E.16.244 & $\mathrm{SP}$ & -- & O0OEXRV \\
\hline 181 & 354246 & 1063212 & Santa Fe National Forest & SP & -- & OOOEXRV \\
\hline 182 & 354329 & 1063324 & Santa Fe National Forest & SP & -- & 110PTOD \\
\hline 183 & 354409 & 1064552 & $17 \mathrm{~N} .02 \mathrm{E} .06 .221$ & GW & -- & $400 \mathrm{PCKB}$ \\
\hline 184 & 354508 & 1064232 & $18 \mathrm{~N} .02 \mathrm{E} .34 .232$ & GW & -- & 110AVIB \\
\hline 185 & $3545 \quad 22$ & 1064200 & $18 \mathrm{~N} .02 \mathrm{E} .26 .334$ & GW & -- & 318ABO L \\
\hline 186 & 354551 & 1061410 & Ramon Vigil Grant Spring 8 & $\mathrm{SP}$ & -- & $112 \mathrm{SNTF}$ \\
\hline 187 & 354551 & 1061424 & Ramon Vigil Grant Spring 9 & SP & -- & $112 \mathrm{SNTF}$ \\
\hline 188 & 354553 & 1061402 & Ramon Vigil Grant Spring 7 & SP & -- & $112 \mathrm{SNTF}$ \\
\hline 189 & 354556 & 1061438 & Ramon Vigil Grant Doe Spring & SP & -- & -- \\
\hline 190 & 354557 & 1061350 & Caja del Rio Grant & $\mathrm{SP}$ & -- & -- \\
\hline
\end{tabular}




$\begin{aligned} & \text { Altitude } \\ & \text { of land- }\end{aligned}$ Total
surface depth
$\begin{gathered}\text { datum of Casing Depth Data Number Period } \\ \text { (feet above well diameter cased avail- of of Water }\end{gathered}$
sea level) (feet) (inches) (feet) able analyses record use
(site name if other than WaTSTORE name)

$$
\begin{aligned}
& \begin{array}{lllllllllll}
-- & -- & - & - & - & \text { A, B } & 1 & 1973 & -- & \text { QW, T1978 (D3). }
\end{array} \\
& \begin{array}{llllllll}
-- & -- & -- & -- & A, B & 2 & 1926-46-- & \text { QW. }
\end{array} \\
& \begin{array}{lllllllll}
-- & -- & -- & -- & A & 1 & 1964 & - & Q W .
\end{array} \\
& \begin{array}{lllllllll}
-- & -- & -- & -- & -- & -- & -- & - & \text { HD. } \\
-- & -- & -- & -- & A, B & 4 & 1945-49 & -- & \text { QW. }
\end{array} \\
& \begin{array}{lllllllll}
-- & -- & -- & -- & -- & -- & -- & -- & \text { GWSI. } \\
-- & -- & -- & -- & A, B, C & 3 & 1973-74 & -- & \text { QW, T1978 (C3), G1982 (VA-34). } \\
-- & -- & -- & -- & A, B & 1 & 1973 & -- & \text { OW, T1978 (D4), G1982 (VA-37). }
\end{array}
\end{aligned}
$$

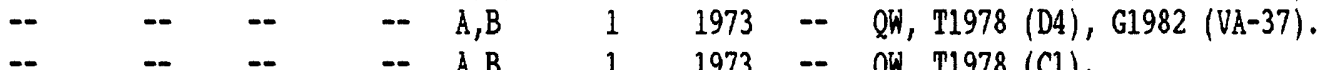

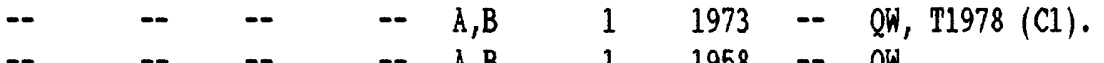

$$
\begin{aligned}
& \begin{array}{llllllllll}
- & - & - & - & - & \text { A,B } & 1 & 1958 & -- & \text { QW. }
\end{array}
\end{aligned}
$$

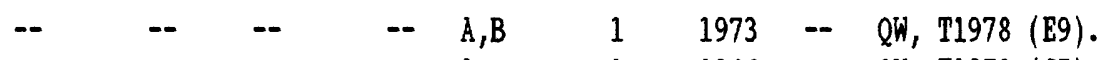

$$
\begin{aligned}
& \begin{array}{llllllllll}
-- & - & - & - & A & 1 & 1946 & -- & \text { QW, T1978 (C5). }
\end{array}
\end{aligned}
$$

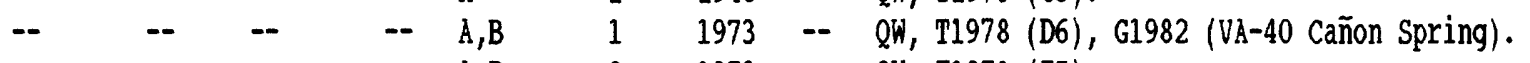

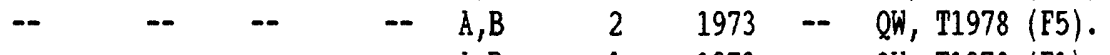

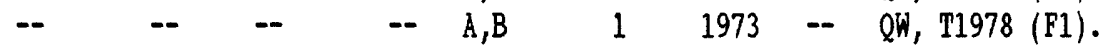

$$
\begin{aligned}
& \begin{array}{lllllllllll}
-- & -- & - & - & - & A, B & 1 & 1973 & - & Q W, T 1978 & \text { (E7). }
\end{array} \\
& \begin{array}{llllllllll}
-- & -- & -- & -- & A, B & 1 & 1973 & -- & Q W, T 1978 & \text { (E6). }
\end{array}
\end{aligned}
$$

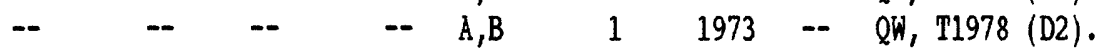

$$
\begin{aligned}
& \begin{array}{llllllllll}
-- & -- & -- & -- & A, B & 1 & 1973 & -- & Q W, T 1978 & \text { (D1). }
\end{array}
\end{aligned}
$$

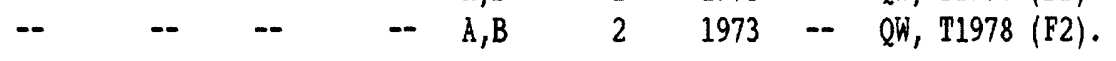

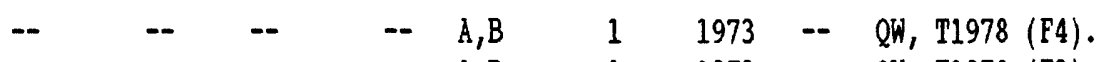

$$
\begin{aligned}
& \begin{array}{llllllllll}
-- & -- & -- & -- & A, B & 1 & 1973 & -- & Q W, T 1978 & \text { (F3). }
\end{array} \\
& \begin{array}{llllllllll}
-- & -- & -- & -- & A, B & 1 & 1973 & -- & Q W, T 1978 & \text { (D5). }
\end{array} \\
& \begin{array}{llllllllll}
-- & -- & -- & -- & A, B & 1 & 1973 & -- & Q W, T 1978 & \text { (H23). }
\end{array}
\end{aligned}
$$

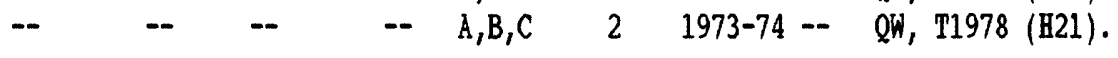

$$
\begin{aligned}
& \begin{array}{llllllllll}
-- & -- & -- & -- & \text { A,B } & 1 & 1963 & -- & \text { QW, T1978 (K16), DOF 1967. } \\
-- & -- & -- & -- & \text { A,B } & 1 & 1963 & -- & \text { QW, T1978 (K14), J1967 (S18.7.30.124), DOF } 1967 .
\end{array}
\end{aligned}
$$

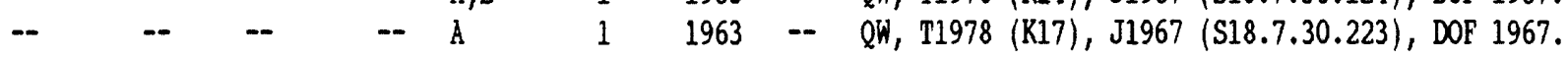

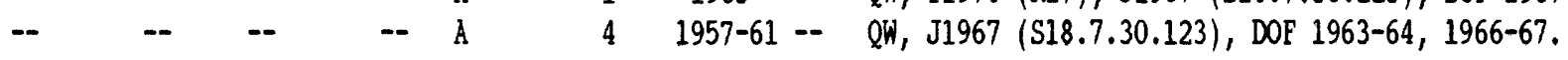

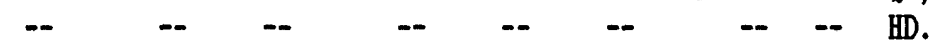


Number

on Lati- Longi-

plate 2 tude

Local identifier

Type owner

Principal

aquifer

$\begin{array}{lllllll}191 & 35 & 45 & 59 & 106 & 13 & 39\end{array}$

$\begin{array}{lllllll}192 & 35 & 46 & 04 & 106 & 41 & 36\end{array}$

$193 \quad 3546 \quad 10 \quad 10613 \quad 16$

$194 \quad 35 \quad 46 \quad 10 \quad 106 \quad 46 \quad 45$

$\begin{array}{lllllll}195 & 35 & 46 & 13 & 106 & 41 & 32\end{array}$

$\begin{array}{lllllll}196 & 35 & 46 & 14 & 106 & 41 & 29\end{array}$

$\begin{array}{lllllll}197 & 35 & 46 & 18 & 106 & 41 & 26\end{array}$

$\begin{array}{lllllll}198 & 35 & 46 & 19 & 106 & 41 & 25\end{array}$

$\begin{array}{lllllll}199 & 35 & 46 & 21 & 106 & 41 & 24\end{array}$

$200 \quad 3546 \quad 23 \quad 106 \quad 41 \quad 21$

$201 \quad 35 \quad 46 \quad 30 \quad 106 \quad 13 \quad 39$

$202 \quad 35 \quad 46 \quad 36 \quad 106 \quad 36 \quad 13$

$203 \quad 35 \quad 46 \quad 38 \quad 106 \quad 36 \quad 15$

$\begin{array}{lllllll}204 & 35 & 47 & 26 & 106 & 46 & 53\end{array}$

$\begin{array}{lllllll}205 & 35 & 47 & 28 & 106 & 39 & 40\end{array}$

$\begin{array}{lllllll}206 & 35 & 47 & 28 & 106 & 41 & 09\end{array}$

$\begin{array}{lllllll}207 & 35 & 47 & 29 & 106 & 41 & 10\end{array}$

$\begin{array}{lllllll}208 & 35 & 47 & 30 & 106 & 41 & 11\end{array}$

$\begin{array}{lllllll}209 & 35 & 48 & 05 & 106 & 40 & 53\end{array}$

$210 \quad 3548 \quad 08 \quad 106 \quad 40 \quad 50$

$\begin{array}{lllllll}211 & 35 & 48 & 13 & 106 & 11 & 48\end{array}$

$212 \quad 3548 \quad 16 \quad 106 \quad 40 \quad 49$

$213 \quad 3548 \quad 18 \quad 106 \quad 1135$

$\begin{array}{lllllll}214 & 35 & 48 & 44 & 106 & 17 & 09\end{array}$

$\begin{array}{lllllll}215 & 35 & 49 & 02 & 106 & 47 & 14\end{array}$

$216 \quad 35 \quad 49 \quad 12 \quad 106 \quad 37 \quad 36$

$\begin{array}{lllllll}217 & 35 & 49 & 15 & 106 & 39 & 43\end{array}$

$\begin{array}{llllllll}218 & 35 & 49 & 17 & 106 & 40 & 41\end{array}$

$\begin{array}{llllllll}219 & 35 & 49 & 18 & 106 & 17 & 20\end{array}$

$\begin{array}{lllllll}220 & 35 & 49 & 26 & 106 & 23 & 18\end{array}$
Caja del Rio Grant

Cañon de San Diego Grant Jenez Hot Springs

Ramon Vigil Grant

Cañon de San Diego Grant

Cañon de San Diego Grant Jenez Hot Springs

18N.02E.23.432

Cañon de San Diego Grant

Cañon de San Diego Grant Jenez Hot Springs

Cañon de San Diego Grant Soda Sp, Jenez Hot Sp

Cañon de San Diego Grant Original Jenez Hot $S p$

Ramon Vigil Grant Ancho Spring

18 N.03E. 22.412

18N.03E. 36.444

Cañon de San Diego Grant Butterfly Sp

Cañon de San Diego Grant

Cañon de San Diego Grant

18N.02E.14.442

Cañon de San Diego Grant Soda Dan Springs West

18N.02E. 12.340

Cañon de San Diego Grant

Ramon Vigil Grant

Cañon de San Diego Grant

Ramon Vigil Grant Spring 4

18N.06R.03.443 Deep Test 9

$18 \mathrm{~N} .01 \mathrm{E} .01 .321$

18N.03E.04.321

Cañon de San Diego Grant

07N.02E.13.441

8N.03E.03.241 D. Test 10

Santa Fe National Forest Sawyer Spring

\section{SP}

SP

SP

SP

SP

GW

SP

SP

SP

SP

SP

SP

$G W$

SP

SP

SP

GW

SP

GW

SP

SP

SP

$\mathrm{SP}$

GW

GW

SP

SP

$\mathrm{GW}$

GW

SP

$--$

-

$-$

112BDLR

110AVIB

325IDER

110AVIB

$-$

$-$

$\ddot{-}$

$\overline{-}$

--

$-$

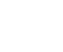

OOOEXRV

112BDLR

--

325KDER

--

110AVIB

325KDER

112 SNTF

325KDER

112 SNTF

$-$

25NDER

110AVIBB

325 MDER

112 SNTF

-.

112TSRG 


$\begin{aligned} & \text { Altitude } \\ & \text { of land- }\end{aligned}$ Total
surface depth
datum of Casing Depth Data Number Period
(feet above well diameter cased avail- of of Water
sea level) (feet) (inches) (feet) able analyses record use
(site name if other than WATSTORE name)

$$
\begin{aligned}
& \begin{array}{llllllllll}
-- & -- & -- & -- & -- & -- & -- & -- & \text { HD. }
\end{array}
\end{aligned}
$$

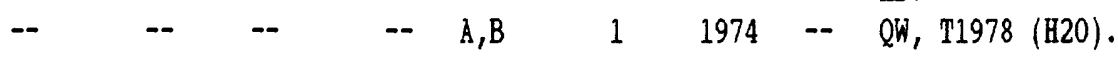

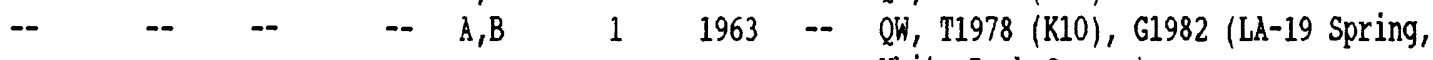

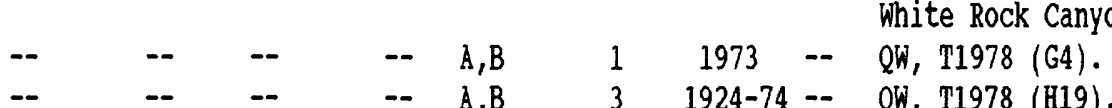

$$
\begin{aligned}
& \begin{array}{lllllllllll}
-- & -- & -- & -- & A, B & 1 & 1974 & -- & Q W, T 1978 & \text { (H17). }
\end{array} \\
& \begin{array}{lllllllll}
-- & -- & -- & -- & A, C & 1 & 1974 & -- & Q W .
\end{array} \\
& \begin{array}{lllllllllll}
-- & -- & -- & -- & A & 2 & 1975 & -- & Q W .
\end{array} \\
& \begin{array}{llllllllll}
-- & -- & -- & -- & A, B & 2 & 1949-51 & -- & Q W .
\end{array}
\end{aligned}
$$

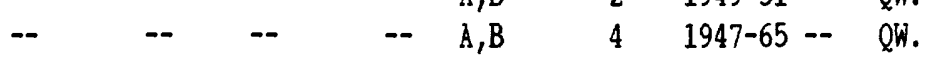

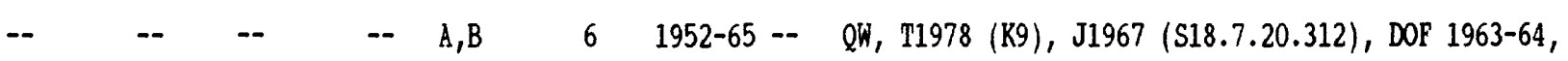

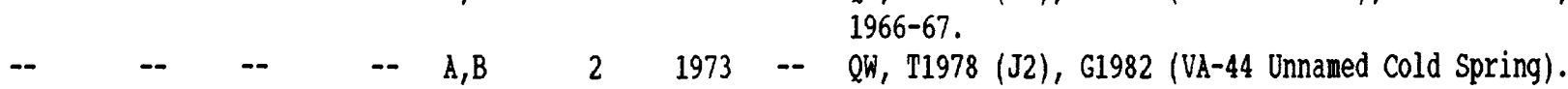

$$
\begin{aligned}
& \begin{array}{llllllllll}
-- & -- & -- & -- & \mathrm{A}, \mathrm{B} & 4 & 1974 & -- & \mathrm{QW} .
\end{array}
\end{aligned}
$$

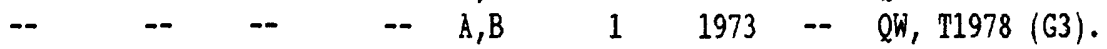

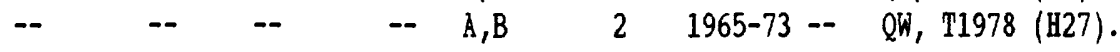

$$
\begin{aligned}
& \begin{array}{lllllllllll}
-- & -- & -- & -- & A, B, C & 1 & 1975 & -- & Q W .
\end{array} \\
& \text {-- } \quad \text {-- } \quad--\quad \quad--\quad A, B, C \quad 3 \quad 1973-74--\quad \text { QW, T1978 (H6), G1982 (VA-6 Soda Dam Spring). }
\end{aligned}
$$

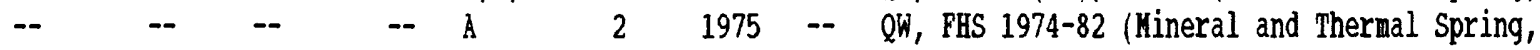

$$
\begin{aligned}
& \begin{array}{llllllllll} 
& & & & & & & \multicolumn{3}{c}{\text { Soda Dam JF-5). }} \\
-- & -- & -- & -- & A & 1 & 1973 & -- & \text { QW, T1978 (H5). } \\
-- & -- & -- & -- & A, B & 3 & 1973-74 & -- & Q W, T 1978 \text { (H4). }
\end{array}
\end{aligned}
$$

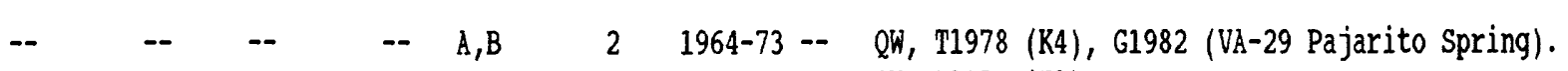

$$
\begin{aligned}
& \begin{array}{lllllllll}
-- & -- & -- & -- & A, B & 2 & 1973-74 & -- & Q W, T 1978 \text { (B3). }
\end{array}
\end{aligned}
$$

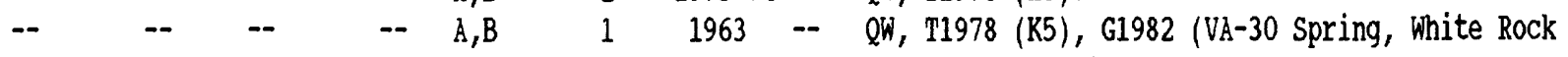

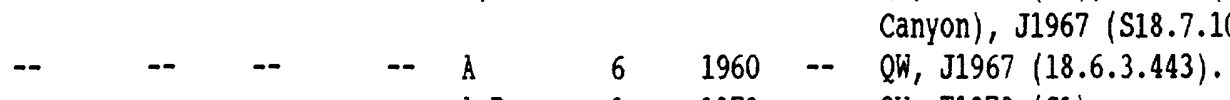

$$
\begin{aligned}
& \begin{array}{llllllllll}
-- & -- & -- & -- & A & 6 & 1960 & -- & Q W, \\
-- & -- & -- & -- & A, B & 1 & 1973 & -- & Q W, T 1978 \text { (G1). }
\end{array} \\
& \text {-- } \quad \text { - } \quad \text {-- } \quad \text {-- } \quad \text { A,B } 5 \text { 1947-74 -- } \quad \text { QW, T1978 (H39), G1982 (VA-3 McCauley Spring), } \\
& \begin{array}{rrrrrrrrll} 
& & & & & & & & \text { FHS 1975-82 (HCCauley Spring RV-5). } \\
-- & -- & -- & -- & \text { A,B } & 2 & 1974 & -- & \text { QW, T1978 (H28). } \\
-- & -- & -- & -- & \text { A,B } & 1 & 1968 & -- & \text { QW. } \\
-- & -- & -- & -- & -- & -- & -- & -- & \text { HD, J1967 (18.6.3.241), DOF 1964, } 1967 . \\
-- & -- & -- & -- & A & 1 & 1961 & -- & Q W, J 1967 \text { (S18.5.02.131). }
\end{array}
\end{aligned}
$$


Number

on Lati- Longi-

plate 2 tude tude

Local identifier

$\begin{array}{llllllll}221 & 35 & 49 & 43 & 106 & 38 & 58 & \text { 19N.03E.32. Test Hole } 1 \\ 222 & 35 & 49 & 43 & 106 & 38 & 58 & \text { 19N.03E.32. Test Hole 2 } \\ 223 & 35 & 49 & 46 & 106 & 38 & 46 & \text { Cañon de San Diego Grant }\end{array}$

$224 \quad 354949 \quad 106 \quad 38 \quad 56$

$\begin{array}{lllllll}225 & 35 & 49 & 49 & 106 & 38 & 56\end{array}$

$226 \quad 354949 \quad 1063857 \quad$ Cañon de San Diego Grant

$227 \quad 354952 \quad 10638 \quad 34$

$\begin{array}{lllllll}228 & 35 & 49 & 52 & 106 & 41 & 11\end{array}$

$\begin{array}{lllllll}229 & 35 & 50 & 13 & 106 & 22 & 57\end{array}$

$\begin{array}{lllllll}230 & 35 & 50 & 14 & 106 & 22 & 55\end{array}$

$\begin{array}{lllllll}231 & 35 & 50 & 28 & 106 & 10 & 17\end{array}$

$\begin{array}{lllllll}232 & 35 & 50 & 40 & 106 & 23 & 10\end{array}$

$\begin{array}{lllllll}233 & 35 & 50 & 40 & 106 & 23 & 12\end{array}$

$\begin{array}{lllllll}234 & 35 & 50 & 48 & 106 & 38 & 24\end{array}$

$\begin{array}{lllllll}235 & 35 & 50 & 49 & 106 & 38 & 09\end{array}$

236

$237 \quad 355130 \quad 1063902$

$\begin{array}{lllllll}238 & 35 & 51 & 34 & 106 & 13 & 32\end{array}$

$\begin{array}{lllllll}239 & 35 & 51 & 58 & 106 & 11 & 42\end{array}$

$240 \quad 3552 \quad 01 \quad 10611 \quad 24$

$241 \quad 355204 \quad 106 \quad 11 \quad 31$

$\begin{array}{lllllll}242 & 35 & 52 & 08 & 106 & 16 & 13\end{array}$

$243 \quad 35 \quad 52 \quad 13 \quad 106 \quad 1300$

$\begin{array}{lllllll}244 & 35 & 52 & 14 & 106 & 12 & 53\end{array}$

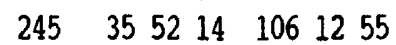

$\begin{array}{lllllll}246 & 35 & 52 & 17 & 106 & 54 & 57\end{array}$

$\begin{array}{lllllll}247 & 35 & 52 & 19 & 106 & 26 & 47\end{array}$

$248 \quad 3552 \quad 20 \quad 106 \quad 1100$

$\begin{array}{lllllll}249 & 35 & 52 & 21 & 106 & 16 & 04\end{array}$

$\begin{array}{lllllll}250 & 35 & 52 & 22 & 106 & 37 & 22\end{array}$

19N.03E.32.324
19N.03E.32.331

19N.03E. 32.331

07N.02E.13.323

Santa Fe National Forest

Santa Fe National Forest American Sp

19N.07E.35.121 Sandia Canyon Spring

Santa Fe National Forest Arnstead Spring

Santa Fe National Forest

19N.03E.29.413

19N.03E. 29.420

19N.03E.28.143 Spence Sp

Baca Location No. 1

19N.07E.20.341

19N.07E.22.131 Basalt Spring

19N.07E.22.114 Los Alamos Spring

19N.07E.22.114 LOS Alamos 4

19N.06E.23.200

$19 \mathrm{~N} .07 \mathrm{E} .20 .221$

19N.07E.20.221 Test Well 1-A

19N.07E.20.221 Test Well 1

19N.01W.14.3332 JM OBS at La Ventana N. Hex. Baca Location No. 1 Valle Grande Sp

19N.07E.15.434 Los Alamos 5

19N.06E.13.344 Test Well 3

19N.03E.16.444
$\mathrm{GW}$

GW

SP

GH

GW

SP

$S P$

GW

SP

$S P$

$\mathrm{SP}$

SP

SP

$S P$

$S P$

$\mathrm{SP}$

SP

SP

GW

SP

SP

GI

GW

GW

GW

GW

GW

$\mathrm{SP}$

GW

GW

GW
Principal

aquifer

Type owner $\begin{gathered}\text { Principal } \\ \text { aquifer }\end{gathered}$

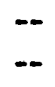

325IDER

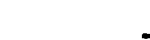

$\begin{array}{ll}-- & \text { 325IDER } \\ -- & 325 \mathrm{MDER}\end{array}$

$-$

318ABO L

325MDER

$-$

$-$

$-$

112TSRG

112TSRG

121TSUQ

121TCCM

$121 \mathrm{TCCH}$

$318 \mathrm{ABO} \mathrm{L}$

318ABO L

112VLLS

112BDLR

Dept. of Energy 112SNTF

San Ildefonso

--

$\mathrm{P}$

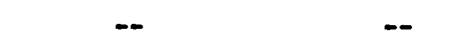

Dept. of Energy

121TSUQ

Dept. of Energy

110AVYB

Dept. of

-.

-- 22111RSN

-- 112VLLS

Dept. of Energy 121TSUQ

$-$

110AVIB 


\begin{tabular}{|c|c|c|c|c|c|c|c|c|}
\hline $\begin{array}{l}\text { Altitude } \\
\text { of land- } \\
\text { surface } \\
\text { datum } \\
\text { (feet above } \\
\text { sea level) }\end{array}$ & $\begin{array}{l}\text { Total } \\
\text { depth } \\
\text { of } \\
\text { well } \\
\text { (feet) }\end{array}$ & $\begin{array}{l}\text { Casing } \\
\text { diameter } \\
\text { (inches) }\end{array}$ & $\begin{array}{l}\text { Depth } \\
\text { cased } \\
\text { (feet) }\end{array}$ & $\begin{array}{l}\text { Data } \\
\text { avail- } \\
\text { able }\end{array}$ & $\begin{array}{l}\text { Number } \\
\text { of } \\
\text { analyses }\end{array}$ & $\begin{array}{l}\text { Period } \\
\text { of } \\
\text { record }\end{array}$ & $\begin{array}{l}\text { Water } \\
\text { use }\end{array}$ & $\begin{array}{l}\text { Source of data and additional data sources } \\
\text { (site name if other than WATSTORE name) }\end{array}$ \\
\hline
\end{tabular}

\begin{tabular}{|c|c|c|c|c|c|c|c|c|}
\hline- & -- & -- & -- & A & 1 & 1975 & - & QW. \\
\hline-- & -- & -- & -- & A & 1 & 1975 & -- & QW. \\
\hline-- & -- & -- & -- & $A, B, C$ & 15 & $1949-75$ & -- & $\begin{array}{l}\text { QW, T1978 (H32), G1982 (VA-31, VA-50 Cold } \\
\text { Mineral Spring), FHS 1974-82 (Mineral Spring } \\
\text { west of Battleship Rock JF-1). }\end{array}$ \\
\hline-- & 155 & -- & -- & $A, B, C$ & 2 & $1949-74$ & -- & QW. \\
\hline-- & -- & -- & -- & A & 1 & 1974 & -- & QW, T1978 (H30). \\
\hline-- & - & - & - & $A, B$ & 1 & 1973 & -- & QW, T1978 (H31). \\
\hline-- & -- & -- & -- & $A, B$ & 2 & 1973 & -- & QW, T1978 (H35). \\
\hline-- & -- & -- & -- & A & 1 & 1969 & -- & QW. \\
\hline-- & -- & -- & -- & A & 1 & 1958 & -- & QW. \\
\hline-- & -- & -- & -- & A & 2 & $1958-61$ & -- & QW, T1978 (R7), J1967 (S19.5.35.114). \\
\hline-- & - & -- & - & A & 3 & 1959-61 & - & QW, T1978 (K19), J1967 (S19.7.35.121), DOF 1963-64. \\
\hline- & - & -- & -- & A & 2 & $1958-61$ & -- & QW, T1978 (R6), J1967 (S19.5.26.322). \\
\hline-- & -- & -- & -- & A & 2 & $1958-61$ & -- & QW. \\
\hline-- & -- & -- & -- & $A, B$ & 2 & 1973 & -- & QW, T1978 (H40). \\
\hline-- & -- & -- & -- & $A, B$ & 2 & $1973-74$ & -- & QW, T1978 (H41). \\
\hline-- & -- & - & -- & $A, B$ & 4 & $1947-73$ & -- & $\begin{array}{l}\text { QW, T1978 (H42), G1982 (VA-1), FHS 1975-82, } \\
\text { (Spence Spring RV-4). }\end{array}$ \\
\hline-- & -- & -- & -- & $A, B$ & 1 & 1973 & -- & QW, T1978 (N16). \\
\hline 6,520 & 2,499 & 12.00 & 2,499 & $A, D$ & 1 & 1965 & $\mathrm{p}$ & QW, GWSI. \\
\hline 6,000 & - & -- & - & $A, B$ & 38 & $1951-65$ & $\mathrm{U}$ & $\begin{array}{l}\text { QW, GWSI, G1982 (LA-5), J1967 (S19.7.22.131), } \\
\text { DOF 1962-66. }\end{array}$ \\
\hline-- & -- & -- & -- & A & 17 & $1957-61$ & -- & QW, J1967 (S19.7.22.114), DOF 1962-65. \\
\hline 5,975 & 1,965 & 10.00 & 1,965 & $A, B, D$ & 36 & $1951-61$ & $\mathrm{P}$ & QW, GWSI, J1967 (19.7.22.114), DOF 1962-67. \\
\hline-- & - & -- & - & $A, B$ & 1 & 1962 & -- & QW. \\
\hline 6,371 & 642 & 6.00 & 642 & $A, C, D$ & 5 & $1952-57$ & $\mathrm{U}$ & QW, GWSI. \\
\hline- & - & -- & -- & $A, B$ & 96 & $1951-63$ & -- & QW, J1967 (19.7.20.221a), DOF 1962-65. \\
\hline-- & -- & -- & -- & $A, B$ & 57 & $1952-61$ & -- & QW, J1967 (19.7.20.221), DOF 1962-64, 1966-67. \\
\hline 6,979 & 2,780 & -- & -- & $A, B$ & 1 & 1978 & - & QW. \\
\hline-- & -- & -- & -- & $A, C$ & 1 & 1954 & -- & QW, T1978 (L3). \\
\hline 5,840 & 1,750 & 10.00 & 1,750 & $A, B, D$ & 10 & $1952-61$ & $\mathrm{p}$ & QW, GWSI, J1967 (19.7.15.434), DOF 1962-65, 1967. \\
\hline- & - & -- & - & $A, B$ & 41 & $1952-61$ & - & QW, G1982 (LA-2), J1967 (19.6.13.344), DOF 1962-66. \\
\hline-- & -- & -- & -- & $A, B$ & 1 & 1974 & -- & QW, T1978 (P6). \\
\hline
\end{tabular}




\begin{tabular}{|c|c|c|c|c|c|c|}
\hline $\begin{array}{l}\text { Number } \\
\text { on } \\
\text { plate } 2\end{array}$ & $\begin{array}{l}\text { Lati- } \\
\text { tude }\end{array}$ & $\begin{array}{l}\text { Longi- } \\
\text { tude }\end{array}$ & Local identifier & Type & Owner & $\begin{array}{l}\text { Principal } \\
\text { aquifer }\end{array}$ \\
\hline 251 & $355226^{\circ}$ & 1063831 & 19N.03E.17.431 & $\mathrm{GW}$ & -- & 112BLSP \\
\hline 252 & 355227 & 1061533 & $19 \mathrm{~N} .06 \mathrm{E} .13 .344$ & GW & -- & 11OPTOD \\
\hline 253 & 355234 & 1061331 & 19N.07E.17.322 Pueblo Canyon 11 & GW & -- & -- \\
\hline 254 & 355238 & 1061027 & 19N.07E.14.312 Los Alamos 6 & GW & Dept. of Energy & 121TSUQ \\
\hline 255 & 355238 & 1061333 & 19N.07E.17.321 Pueblo Canyon 10 & GW & $\cdots$ & -- \\
\hline 256 & 355243 & 1061349 & 19N.07E.18.244 PO 1-A & GW & -- & -- \\
\hline 257 & 355247 & 1063836 & Baca Location No. 2 & $\mathrm{SP}$ & -- & 110PTOD \\
\hline 258 & 355251 & 1061358 & 19N.07E.18.241 Pueblo Canyon 9 & GW & -- & -- \\
\hline 259 & 355251 & 1061435 & 19N.07E.18.132 Pueblo Canyon 8 & GW & -- & -- \\
\hline 260 & 355252 & 1061434 & 19N.07E.18.132 PO 3-B & GW & -- & -- \\
\hline 261 & 355252 & 1061450 & 19N.06E.13.242 PO 4-A & GW & -- & -- \\
\hline 262 & 355253 & 1061403 & 19N.07E.18.241 Otowi Seep & SP & -- & -- \\
\hline 263 & 355254 & 1061429 & 19N.07E.18.124 Hamilton Bend Spring & SP & -- & -- \\
\hline 264 & 355254 & 1064012 & $19 \mathrm{~N} .02 \mathrm{E} .13 .242$ & GW & -- & 400PCYB \\
\hline 265 & 355254 & 1064012 & 19N.02E.13.242 & GW & -- & $400 \mathrm{PCHB}$ \\
\hline 266 & 355254 & 1064012 & 19N.02E.13.242 & GW & -- & 400PCIB \\
\hline 267 & 355254 & 1064012 & 19N.02E.13.242 & GW & $\cdots$ & 400РCKB \\
\hline 268 & 355257 & 1061452 & 19N.06E.13.242 PO 4-B & GW & -- & -- \\
\hline 269 & 355300 & 1060923 & 19N.07E.13.114 Los Alamos 1B & GW & -- & -- \\
\hline 270 & 355300 & 1061549 & 19N.06E.13.131 Pueblo Canyon 7 & GW & -- & -- \\
\hline 271 & 355301 & 1060922 & 19N.07E.13.114 Los Alamos 1 & GW & -- & -- \\
\hline 272 & 355303 & 1060946 & 19N.07E.14.221 Los Alamos 3 & GW & Dept. of Energy & -- \\
\hline 273 & 355304 & 1060900 & 19N.07E.13.211 & GW & Dept. of Energy & -- \\
\hline 274 & 355304 & 1060922 & 19N.07E.13.112 Indian Spring & SP & -- & -- \\
\hline 275 & 355304 & 1060936 & 19N.07E.14.222 Los Alamos 2 & GW & -- & -- \\
\hline 276 & 355305 & 1061614 & 19N.06E.14.221 Test Well 2 & GW & -- & -- \\
\hline 277 & 355305 & 1061616 & 19N.06E.14.221 Test Well 2-A & GW & -- & -- \\
\hline 278 & 355305 & 1061824 & 19N.06E.16.212 Acid Canyon 2 & GW & -- & -- \\
\hline 279 & 355307 & 1061613 & 19N.06E.14.222 Pueblo Canyon 6 & GW & -- & -- \\
\hline 280 & 355307 & 1061825 & 19N.06E.16.212 Acid Canyon 3 & GW & -- & -- \\
\hline
\end{tabular}




\begin{tabular}{|c|c|c|c|c|c|c|c|c|}
\hline $\begin{array}{l}\text { Altitude } \\
\text { of land- } \\
\text { surface } \\
\text { datum } \\
\text { (feet above } \\
\text { sea level) }\end{array}$ & $\begin{array}{c}\text { Total } \\
\text { depth } \\
\text { of } \\
\text { well } \\
\text { (feet) }\end{array}$ & $\begin{array}{l}\text { Casing } \\
\text { diameter } \\
\text { (inches) }\end{array}$ & $\begin{array}{l}\text { Depth } \\
\text { cased } \\
\text { (feet) }\end{array}$ & $\begin{array}{l}\text { Data } \\
\text { avail- } \\
\text { able }\end{array}$ & $\begin{array}{c}\text { Number } \\
\text { of } \\
\text { analyses }\end{array}$ & $\begin{array}{l}\text { Period } \\
\text { of } \\
\text { record }\end{array}$ & $\begin{array}{l}\text { Water } \\
\text { use }\end{array}$ & $\begin{array}{l}\text { Source of data and additional data sources } \\
\text { (site name if other than WATSTORE name) }\end{array}$ \\
\hline-- & -- & - & - & $\mathrm{A}, \mathrm{B}$ & 3 & 1973 & - & $\begin{array}{l}\text { QW, T1978 (P7), FHS 1974-82 (Hofein's Flowing } \\
\text { Well L-27). }\end{array}$ \\
\hline- & - & - & - & $\AA$ & 1 & 1965 & -- & QW. \\
\hline-- & - & -- & -- & A & 3 & 1959 & -- & QW, J1967 (19.7.17.322), DOF 1962-65. \\
\hline 5,770 & 1,790 & 10.00 & 1,790 & $A, B, D$ & 57 & $1951-65$ & $\mathrm{p}$ & $\begin{array}{l}\text { QW, GWSI, G1982 (LA-6), J1967 (19.7.14.312), } \\
\text { DOF 1962-67. }\end{array}$ \\
\hline- & - & - & -- & A & 16 & $1954-61$ & - & QW, J1967 (19.7.17.321), DOF 1962-65. \\
\hline- & -- & -- & -- & A & 8 & $1957-58$ & -- & QW, J1967 (19.7.18.244). \\
\hline-- & - & -- & -- & $A, B$ & 3 & $1959-74$ & -- & QW, T1978 (P7). \\
\hline-- & -- & -- & -- & A & 15 & $1954-59$ & -- & QW, J1967 (19.7.18.241a), DOF 1963-65. \\
\hline-- & -- & -- & -- & A & 16 & $1954-59$ & -- & QW, J1967 (19.7.18.132), DOF 1962. \\
\hline-- & -- & -- & -- & A & 18 & $1957-61$ & -- & QW, J1967 (S19.7.18.132), DOF 1962, 1964-65. \\
\hline-- & -- & -- & -- & A & 13 & $1957-61$ & -- & QW, J1967 (19.6.13.242a), DOF 1962, 1964-65. \\
\hline- & -- & - & - & $A, B$ & 37 & $1951-61$ & -- & QW, J1967 (S19.7.18.241), DOF 1963-65. \\
\hline-- & -- & -- & -- & $A$ & 27 & $1955-61$ & -- & QW, J1967 (S19.7.18.124), DOF 1962-65. \\
\hline- & -- & -- & -- & $A, B$ & 2 & 1974 & -- & QW. \\
\hline-- & -- & -- & -- & $A, B$ & 3 & 1974 & -- & QW. \\
\hline-- & -- & -- & -- & $A, B$ & 1 & 1974 & -- & QW. \\
\hline-- & - & -- & -- & $A, B$ & 1 & 1974 & -- & QW. \\
\hline-- & - & -- & -- & $A$ & 11 & $1957-61$ & -- & QW, J1967 (19.6.13.242b), DOF 1964. \\
\hline 5,625 & 1,750 & 10.00 & 1,750 & A & 10 & $1960-61$ & $\mathrm{P}$ & $\begin{array}{l}\text { QW, GWSI, G1982 (LA-7), J1967 (19.7.13.114b), } \\
\text { DOF 1962-67. }\end{array}$ \\
\hline-- & -- & -- & -- & A & 18 & $1954-61$ & -- & QW, J1967 (19.6.13.131), DOF 1962. \\
\hline-- & -- & -- & -- & A & 10 & $1951-60$ & -- & QW, T1978 (T8), J1967 (19.7.13.114). \\
\hline 5,672 & 870 & 12.00 & 870 & $A, B$ & 18 & $1951-61$ & $P$ & $\begin{array}{l}\text { QW, GWSI, T1978 (T9), J1967 (19.7.14.221), } \\
\text { DOF 1962-67. }\end{array}$ \\
\hline 5,620 & 475 & -- & -- & -- & -- & -- & U & GWSI. \\
\hline- & -- & -- & -- & A & 8 & $1957-61$ & -- & QW，T1977 (T7)，J1967 (S19.7.13.112), DOF 1962-64. \\
\hline- & -- & -- & -- & A & 6 & $1957-61$ & -- & QW, T1978 (T10), J1967 (19.7.14.222), DOF 1962-66. \\
\hline-- & -- & -- & -- & $A, B$ & 52 & $1951-61$ & -- & $\begin{array}{l}\text { QW, G1982 (LA-3), J1967 (19.6.14.221), } \\
\text { DOF 1962-65. }\end{array}$ \\
\hline-- & -- & - & -- & $A, B$ & 54 & $1949-64$ & -- & QW, T1978 (S3), J1967 (19.6.14.221a), DOF 1962-65. \\
\hline-- & - & -- & -- & A & 2 & $1954-57$ & -- & QW, J1967 (19.6.16.212), DOF 1962. \\
\hline-- & -- & - & -- & A & 22 & $1954-61$ & -- & QW, J1967 (19.6.14.222), DOF 1962-65. \\
\hline-- & -- & -- & -- & A & 11 & $1954-59$ & -- & QW, J1967 (19.6.16.212), DOF 1962-64. \\
\hline
\end{tabular}




\begin{tabular}{|c|c|c|c|c|c|c|}
\hline $\begin{array}{l}\text { Number } \\
\text { on } \\
\text { plate } 2\end{array}$ & $\begin{array}{l}\text { Lati- } \\
\text { tude }\end{array}$ & $\begin{array}{l}\text { Longi- } \\
\text { tude }\end{array}$ & Local identifier & Type & Owner & $\begin{array}{l}\text { Principal } \\
\text { aquifer }\end{array}$ \\
\hline 281 & 355309 & 1061658 & 19N.06E.11.333 Pueblo Canyon 5 & GW & -- & -- \\
\hline 282 & 355312 & 1061827 & 19N.06E.09.434 Acid Canyon 4 & GW & -- & -- \\
\hline 283 & 355313 & 1064933 & 18N.01W.1122 Elk Spring & SP & -- & 211DKOT \\
\hline 284 & 355313 & 1065840 & 19N.01W.07.4314 Piedra Lumbre Backup Well & GW & -- & $110 \mathrm{AVNB}$ \\
\hline 285 & 355315 & 1061818 & 19N.06W.09.441 & $\mathrm{SP}$ & -- & -- \\
\hline 286 & 355315 & 1063849 & 19N.03E.08.442 & GH & -- & $110 \mathrm{AVIB}$ \\
\hline 287 & 355316 & 1065958 & 31 N.02W. 24.132 & GW & -- & -- \\
\hline 288 & 355317 & 1061820 & 19N.06E.09.441 Acid Canyon 5 & GW & -- & -- \\
\hline 289 & 355318 & 1061728 & 19N.06E.10.431 Pueblo Canyon 4 & GW & -- & -- \\
\hline 290 & 355318 & 1061758 & 19N.06E.10.331 Pueblo Canyon 3 & GW & -- & -- \\
\hline 291 & 355321 & 1061806 & 19N.06E.09.442 Pueblo Canyon 2 & GW & -- & -- \\
\hline 292 & 355322 & 1061815 & 19N.06E.09.423 Pueblo Canyon 1 & GH & -- & -- \\
\hline 293 & 355333 & 1060859 & 19N.07E.12.233 Sacred Spring & SP & -- & 121TSUQ \\
\hline 294 & 355344 & 1065725 & 19N.01W.08.223 Brandy 1 BLM & $G W$ & -- & 211NENF \\
\hline 295 & 355405 & 1061147 & 19N.07E.04.444 Layne Western Well & GW & -- & 121TSUQ \\
\hline 296 & 355407 & 1061147 & 19N.07E.04.444 Guaje 1 & GW & Dept. of Energy & 121TSUQ \\
\hline 297 & $35 \quad 5412$ & 1061157 & 19N.07E.04.441 Guaje 1-A & GW & Dept. of Energy & 121TSUQ \\
\hline 298 & 355422 & 1061209 & 19N.07E.04.411 Guaje 2 & GW & Dept. of Energy & 121TSUQ \\
\hline 299 & 355423 & 1063656 & Baca Location No. 1 Grant Ladies Bath House & $\mathrm{SP}$ & -- & 112VLLS \\
\hline 300 & 355424 & 1063655 & Baca Location No. 1 Grant Mens Bath House & $\mathrm{SP}$ & - & 112VLLS \\
\hline 301 & 355426 & 1063656 & Baca Location No. 1 Grant Lemonade Sp & $\mathrm{SP}$ & -- & -- \\
\hline 302 & 355426 & 1063700 & Baca Location No. 1 Grant Electric Sp & $\mathrm{SP}$ & -- & -- \\
\hline 303 & 355427 & 1063702 & Baca Location No. 1 Laxative Sp & SP & -- & -- \\
\hline 304 & 355429 & 1063654 & Baca Location No. 1 Grant Footbath Sp & SP & -- & -- \\
\hline 305 & 355429 & 1063654 & Baca Location No. 1 Grant Alum Sp & SP & -- & -- \\
\hline
\end{tabular}




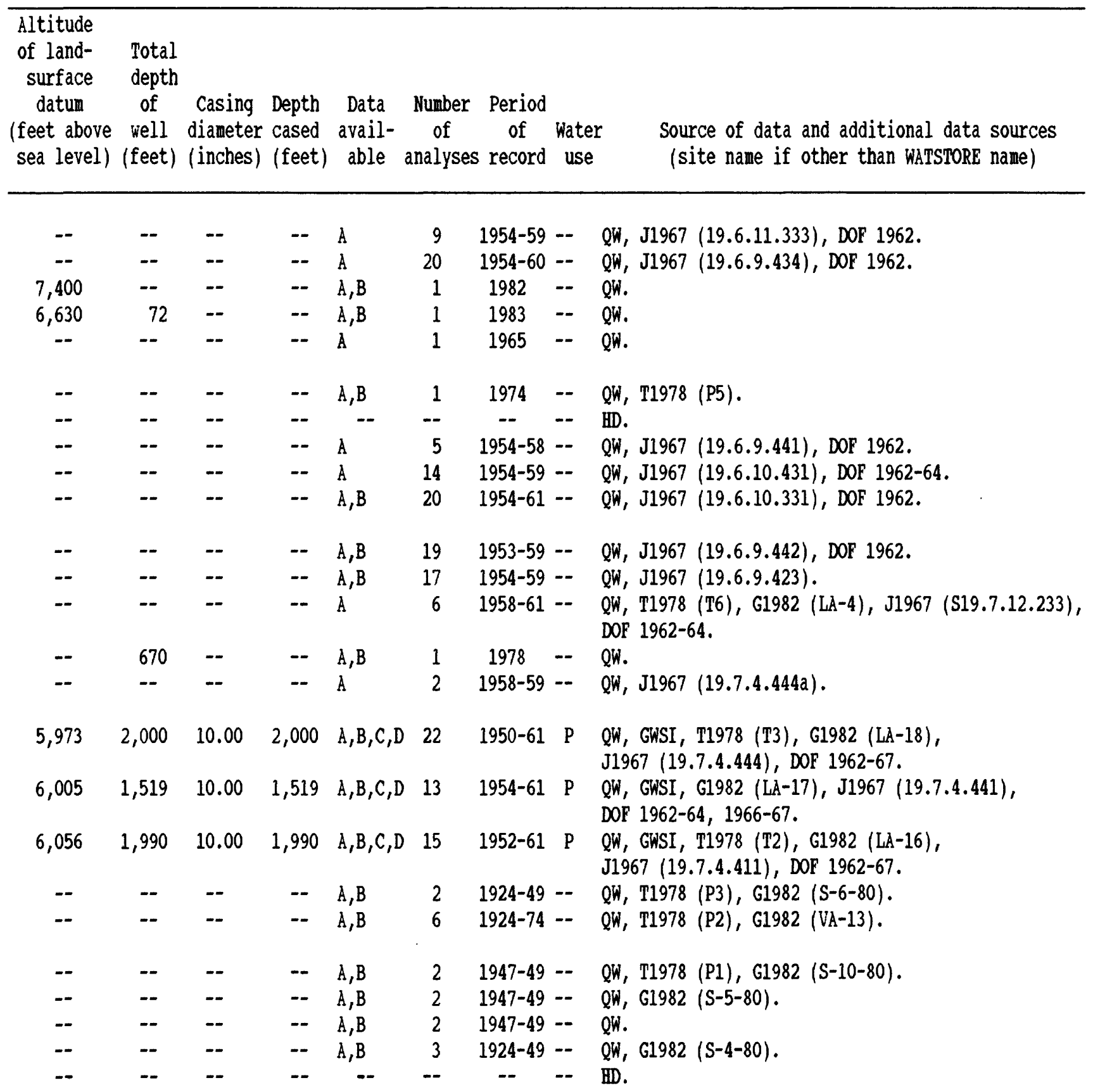


Number

on Lati- Longi-

plate 2 tude tude

Local identifier

Principal

aquifer

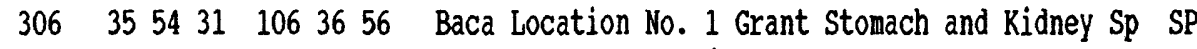

$307 \quad 355432 \quad 1061244 \quad$ 19N.07E.04.133 Guaje 3

GW Dept. of Energy

121TSUQ

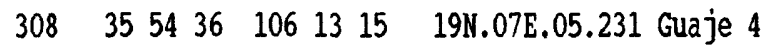

GW Dept. of Energy

121TSUQ

$309 \quad 355450 \quad 1063625$

Baca Location No. 1 Turkey Sp

310

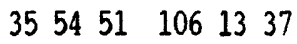

19N.07E.05.112 Guaje 5

SP

GI

Dept. of Energy

121TSUQ

$\begin{array}{lllllll}311 & 35 & 54 & 57 & 106 & 09 & 01\end{array}$

$\begin{array}{lllllll}312 & 35 & 55 & 01 & 106 & 35 & 38\end{array}$

$\begin{array}{lllllll}313 & 35 & 55 & 41 & 106 & 42 & 08\end{array}$

$\begin{array}{lllllll}314 & 35 & 55 & 44 & 106 & 42 & 37\end{array}$

$\begin{array}{lllllll}315 & 35 & 55 & 51 & 106 & 41 & 38\end{array}$

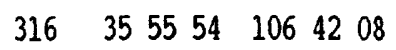

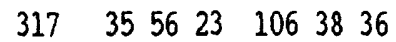

$\begin{array}{lllllll}318 & 35 & 56 & 34 & 106 & 42 & 14\end{array}$

$319 \quad 355650 \quad 106 \quad 38 \quad 31$

$\begin{array}{llllll}320 & 35 & 5733 & 106 & 29 & 13\end{array}$

$\begin{array}{lllllll}321 & 35 & 58 & 16 & 106 & 33 & 41\end{array}$

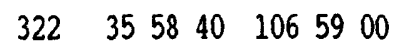

$\begin{array}{lllllll}323 & 35 & 59 & 13 & 106 & 58 & 43\end{array}$

$\begin{array}{lllllll}324 & 35 & 59 & 18 & 106 & 58 & 33\end{array}$

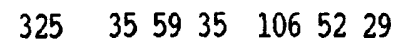

$\begin{array}{lllllll}326 & 35 & 59 & 39 & 106 & 04 & 54\end{array}$

$\begin{array}{lllllll}327 & 36 & 00 & 35 & 106 & 56 & 07\end{array}$

$\begin{array}{lllllll}328 & 36 & 00 & 45 & 106 & 57 & 50\end{array}$

$\begin{array}{lllllll}329 & 36 & 00 & 52 & 106 & 57 & 49\end{array}$

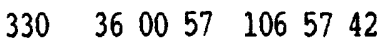

$\begin{array}{lllllll}331 & 36 & 01 & 00 & 106 & 08 & 53\end{array}$

$\begin{array}{lllllll}332 & 36 & 01 & 07 & 106 & 56 & 43\end{array}$

$\begin{array}{lllllll}333 & 36 & 01 & 08 & 106 & 10 & 33\end{array}$

$\begin{array}{lllllll}334 & 36 & 01 & 09 & 106 & 05 & 35\end{array}$

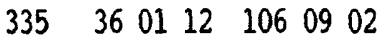

20N.07E. 36.400

Baca Location No. 1 Grant

Santa Fe National Forest

20N.02E. 27.433

20N.02E.26.433

Santa Fe National Forest

Santa Fe National Forest San Antonio Hot Spring

20N.02E. 27.222

Santa Fe National Forest Cold Spring

Baca Location No.1 Grant

Baca Location No. 1 Grant San Antonio Warm Sp

20N.01W.07.32 Brandy 2 BLM

20N.01W.06.432

$20 \mathrm{~N} .01$ W. 06.432

20N.01E.06.233 Horseshoe Spring

20N.08E.03.3221

$21 \mathrm{~N} .01 \% .34 .100$

21N.01พ. 32.211

21N.01W.29.433

21N.01พ.29.432

21N.07E. 25.343

21N.01พ. 28.410

21N.07E. 27.400

21N.08E.28.441

21N.07E.25.314

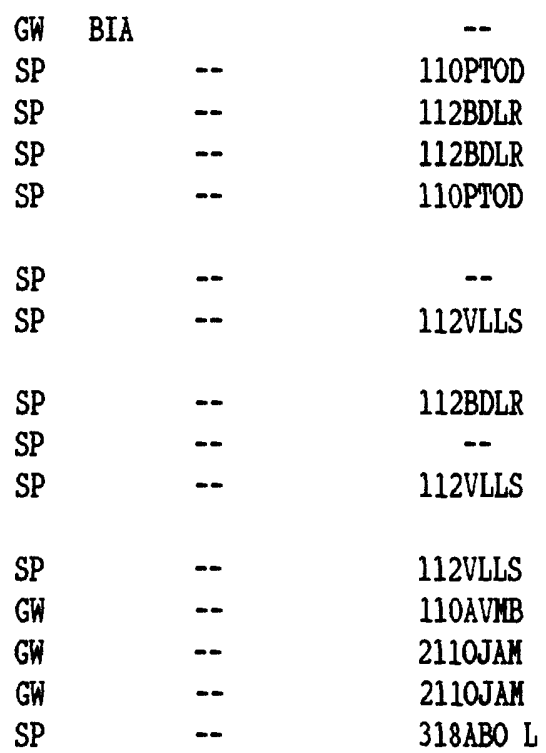

Gh

SP

GW

GW

GW

City of Española

-.

$-$

$-$

$-$

$--$

2110JAM

$\begin{array}{llc}\text { GW } & \text { Reser, Kevin } & -- \\ \text { GW } & -- & \text { 2110JAM } \\ \text { GW } & \text { Green, Charlie C. } & \text { 112SNTP } \\ \text { GW } & \text { Kontoya, Mike } & -- \\ \text { GW } & \text { Valerie, Sam } & -.\end{array}$ 


\begin{tabular}{|c|c|c|c|c|c|c|c|c|}
\hline $\begin{array}{l}\text { Altitude } \\
\text { of land- } \\
\text { surface } \\
\text { datum } \\
\text { (feet above } \\
\text { sea level) }\end{array}$ & $\begin{array}{c}\text { Total } \\
\text { depth } \\
\text { of } \\
\text { well } \\
\text { (feet) }\end{array}$ & $\begin{array}{l}\text { Casing } \\
\text { diameter } \\
\text { (inches) }\end{array}$ & $\begin{array}{l}\text { Depth } \\
\text { cased } \\
\text { (feet) }\end{array}$ & $\begin{array}{l}\text { Data } \\
\text { avail- } \\
\text { able }\end{array}$ & $\begin{array}{l}\text { Number } \\
\text { of } \\
\text { analyses }\end{array}$ & $\begin{array}{l}\text { Period } \\
\text { of } \\
\text { s record }\end{array}$ & $\begin{array}{l}\text { Water } \\
\text { use }\end{array}$ & $\begin{array}{l}\text { Source of data and additional data sources } \\
\text { (site name if other than WATSTORE name) }\end{array}$ \\
\hline-- & -- & -- & -- & $A, B$ & 3 & $1924-49$ & -- & QW. \\
\hline 6,140 & 1,800 & 10.00 & 1,800 & $A, B, C, D$ & 20 & $1950-64$ & $\mathrm{P}$ & $\begin{array}{l}\text { QW, GWSI, G1982 (LA-15), J1967 (19.7.4.133), } \\
\text { DOF 1962-64, 1966-67. }\end{array}$ \\
\hline 6,240 & 1,930 & 10.00 & 1,930 & $A, B, D$ & 11 & $1951-61$ & $\mathrm{P}$ & $\begin{array}{l}\text { QW, GWSI, T1978 (T5), G1982 (LA-14), } \\
\text { J1967 (19.7.5.231), DOF 1962-67. }\end{array}$ \\
\hline-- & -- & -- & -- & $A, B$ & 1 & 1924 & -- & Qh. \\
\hline 6,306 & 1,840 & 10.00 & 1,840 & $A, B, C, D$ & 19 & $1951-61$ & $\mathrm{P}$ & $\begin{array}{l}\text { QW, GWSI, T1978 (T4), G1982 (LA-13), } \\
\text { J1967 (19.7.5.112), DOF 1962-67. }\end{array}$ \\
\hline 5,790 & 225 & -- & -- & D & -- & -- & $S$ & GWSI. \\
\hline- & - & - & - & $A, B$ & 1 & 1949 & - & QW, T1978 (P19), G1982 (VA-22 Bubbling Pool). \\
\hline-- & -- & -- & -- & $A, B$ & 1 & 1974 & -- & QW, T1978 (N13). \\
\hline-- & -- & -- & -- & $\mathrm{A}, \mathrm{B}$ & 1 & 1974 & -- & QW, T1978 (N11). \\
\hline-- & -- & -- & -- & $A$ & 1 & 1973 & -- & QW, T1978 (N9). \\
\hline- & - & -- & -- & A & 2 & $1973-74$ & -- & QW. \\
\hline-- & -- & -- & -- & $A, B, C$ & 3 & $1973-74$ & -- & $\begin{array}{l}\text { QW, T1978 (P12), G1982 (VA-4), FHS 1975-82 } \\
\text { (RV-2). }\end{array}$ \\
\hline-- & -- & - & -- & $A, B, C$ & 4 & $1972-74$ & -- & QW, T1978 (N10), G1982 (VA-47 Unnamed Cold Spring) \\
\hline-- & -- & - & -- & $A, B$ & 1 & 1976 & -- & QW, T1978 (P11). \\
\hline-- & -- & -- & -- & $A, B, C$ & 2 & $1949-54$ & -- & QW, T1978 (Q1). \\
\hline -- & -- & - & -- & A & 2 & $1947-56$ & -- & QW, T1978 (P9), G1982 (VA-20 Bathhouse Spring). \\
\hline- & 260 & - & -- & $\mathrm{A}, \mathrm{B}$ & 1 & 1978 & -- & QW. \\
\hline-- & -- & -- & -- & $\AA$ & 1 & 1959 & -- & QW. \\
\hline -- & - & - & - & A & 1 & 1959 & -- & QW. \\
\hline-- & - & - & -- & $A, B, C$ & 3 & 1974 & - & QW, T1978 (M1). \\
\hline 5,600 & 150 & 15.00 & 150 & $D$ & -- & - & $\mathrm{P}$ & GWSI. \\
\hline- & -- & -- & -- & $A, B$ & 1 & 1944 & -- & QW. \\
\hline- & - & -- & - & $A^{\prime}$ & 1 & 1952 & - & QW. \\
\hline- & - & - & -- & A & 1 & 1956 & -- & QW. \\
\hline-- & -- & - & -- & A & 1 & 1953 & -- & QW. \\
\hline 6,500 & 80 & 6.00 & 80 & D & -- & -- & H & GWSI. \\
\hline-- & -- & -- & -- & $A, B$ & 2 & $1956-57$ & -- & QW. \\
\hline 6,440 & 543 & 5.56 & 543 & D & -- & -- & $S$ & GWSI. \\
\hline 5,765 & 95 & 48.00 & -- & D & -- & -- & $\mathrm{H}$ & GWSI. \\
\hline 6,280 & 55 & 6.63 & 53 & D & - & -- & $\mathrm{H}$ & GWSI. \\
\hline
\end{tabular}




\begin{tabular}{|c|c|c|c|c|c|c|}
\hline $\begin{array}{l}\text { Number } \\
\text { on } \\
\text { plate } 2\end{array}$ & $\begin{array}{l}\text { Lati- } \\
\text { tude }\end{array}$ & $\begin{array}{l}\text { Longi- } \\
\text { tude }\end{array}$ & Local identifier & Type & owner & $\begin{array}{l}\text { Principal } \\
\text { aquifer }\end{array}$ \\
\hline 336 & $\begin{array}{lll}36 & 01 & 17\end{array}$ & 1060529 & $21 \mathrm{~N} .08 \mathrm{E} .28 .424$ & GW & Lowdermilk, Ben & -- \\
\hline 337 & 360118 & 1065725 & 21N.01W.29.244 & GW & -- & 2110JAM \\
\hline 338 & 360120 & 1065659 & $21 \mathrm{~N} .01 \mathrm{~W} .28 .143$ & GW & -- & 2110JAM \\
\hline 339 & 360120 & 1065730 & $21 \mathrm{~N} .01 W .29 .24$ & $G$ & -- & 2110JAM \\
\hline 340 & 360133 & 1065642 & $21 \% .01 \% .28 .213$ & GW & - & 2110JAM \\
\hline 341 & 360207 & 1060906 & $21 \mathrm{~N} .07 \mathrm{E} .24 .310$ & $G W$ & Trujillo, Joe H. & 110AVKB \\
\hline 342 & $3602 \quad 22$ & 1060600 & $21 \mathrm{~N} .08 \mathrm{E} .21 .144$ & GW & Salazar, Ross & - \\
\hline 343 & $36 \quad 0237$ & 1064139 & $21 \mathrm{~N} .02 \mathrm{E} .14 .433$ & SP & -- & 318ABO L \\
\hline 344 & 360249 & 1065438 & 21N.01W.14.413 Martinez Sp & SP & -- & 110AVKB \\
\hline 345 & 360255 & 1065411 & 21N.01W.14.421 & $\mathrm{SP}$ & -- & 110PTOD \\
\hline 346 & 360255 & 1065425 & 21N.01W.14.413 & SP & - & 110AVIB \\
\hline 347 & 360256 & 1065424 & 21N.01พ.14.421 & GW & -- & 110AVIB \\
\hline 348 & 360259 & 1060637 & $21 \mathrm{~N} .08 \mathrm{E} .17 .423$ & GW & Salazar, Roman H. & -- \\
\hline 349 & 360301 & 1061211 & 21N.07E.16.312 & GW & Sancher, Nick & $\rightarrow$ \\
\hline 350 & 360340 & 1060711 & $21 \mathrm{~N} .08 \mathrm{E} .08 .343$ & GW & Consavage, Anthony & 112 SNTF \\
\hline 351 & 360355 & 1060707 & 21N.08E.08.320 & GW & Padilla, Emma & 110AVIB \\
\hline 352 & $36 \quad 0432$ & 1060704 & 21N.08E.05.344 & GW & Trujillo, Arturo $\mathrm{M.}$ & 110AVIB \\
\hline 353 & $\begin{array}{lll}36 & 05 & 01\end{array}$ & 1060723 & $21 \mathrm{~N} .08 \mathrm{E} .05 .130$ & GW & Sanchez, Ben & $110 A V Y B$ \\
\hline 354 & $\begin{array}{lll}36 & 05 & 37\end{array}$ & 1060815 & 22N.07E. 36.422 & GW & Maestas, Pablo & -- \\
\hline 355 & 360546 & 1060823 & 22N.07E. 36.243 & GW & Robertson, Smokey & - \\
\hline 356 & 360553 & 1060859 & 22N.07E. 36.100 & GW & Duran, Max & $110 \mathrm{AVIB}$ \\
\hline 357 & 360603 & 1060848 & 22N.07E. 36.122 & GW & Herrera, Jose D. & -- \\
\hline 358 & 360610 & 1060854 & 22N.07E. 25.343 & GW & Moya, Luis & -- \\
\hline 359 & 360616 & 1060921 & $22 \mathrm{~N} .07 \mathrm{E} .26 .442$ & GW & Maestas, George & 110AVIB \\
\hline 360 & 360652 & 1060821 & 22N.07E. 25.220 & GW & Fernandez & -- \\
\hline 361 & 360652 & 1060939 & 22N.07E. 26.210 & GW & Atencio & 110AVYB \\
\hline 362 & 360656 & 1060918 & $22 \mathrm{~N} .07 \mathrm{E} .26 .222$ & GW & Loper, Antonio & - \\
\hline 363 & 360700 & 1060930 & 22N.07E. 23.443 & GW & Sandoval, Maria & - \\
\hline 364 & 360703 & 1060952 & 22N.07E. 23.344 & GW & Dixon, Theresa L. & - \\
\hline 365 & 360716 & 1060903 & 22N.07E. 24.314 & GW & Walker, Madeline $\mathrm{H}$. & 110AVIB \\
\hline
\end{tabular}




\begin{tabular}{|c|c|c|c|c|c|c|c|c|}
\hline $\begin{array}{l}\text { Altitude } \\
\text { of land- } \\
\text { surface } \\
\text { datum } \\
\text { (feet above } \\
\text { sea level) }\end{array}$ & $\begin{array}{l}\text { Total } \\
\text { depth } \\
\text { of } \\
\text { well } \\
\text { (feet) }\end{array}$ & $\begin{array}{l}\text { Casing } \\
\text { diameter } \\
\text { (inches) }\end{array}$ & $\begin{array}{l}\text { Depth } \\
\text { cased } \\
\text { (feet) }\end{array}$ & $\begin{array}{c}\text { Data } \\
\text { avail- } \\
\text { able }\end{array}$ & $\begin{array}{c}\text { Number } \\
\text { of } \\
\text { analyses }\end{array}$ & $\begin{array}{l}\text { Period } \\
\text { of } \\
\text { record }\end{array}$ & $\begin{array}{l}\text { Water } \\
\text { use }\end{array}$ & $\begin{array}{l}\text { Source of data and additional data sources } \\
\text { (site name if other than WATSTORE name) }\end{array}$ \\
\hline 5,730 & 350 & 8.00 & 300 & D & - & -- & H & GWSI. \\
\hline -- & - & -- & -- & A & 1 & 1944 & -- & QW. \\
\hline-- & -- & -- & -- & $A, B, C$ & 2 & $1957-59$ & -- & QW. \\
\hline-- & - & -- & -- & -- & - & -- & -- & $\mathrm{HD}$. \\
\hline-- & -- & -- & -- & $A, B$ & 2 & $1957-58$ & - & QW. \\
\hline 6,240 & 37 & 6.63 & 37 & D & -- & -- & H & GWSI. \\
\hline 5,680 & 100 & 48.00 & 50 & D & $\cdots$ & $\cdots$ & H & GHSI. \\
\hline- & -- & -- & -- & $A, B, C$ & 3 & 1974 & -- & QW, T1978 (U1). \\
\hline-- & - & -- & -- & $A$ & 1 & 1959 & -- & QW. \\
\hline-- & -- & -- & -- & $\mathrm{A}, \mathrm{B}$ & 1 & 1965 & -- & QW. \\
\hline-- & - & -- & -- & A & 1 & 1959 & -- & QW. \\
\hline-- & -- & -- & -- & A & 1 & 1965 & - & QW. \\
\hline 5,680 & 100 & 6.63 & 96 & D & -- & -- & H & GWSI. \\
\hline 6,345 & 264 & 6.63 & 264 & D & -- & -- & H & GWSI. \\
\hline 5,710 & 79 & 6.63 & 79 & D & -- & -- & $\mathrm{H}$ & GWSI. \\
\hline 5,680 & 55 & 6.63 & 55 & D & -- & -- & H & GWSI. \\
\hline 5,680 & 48 & 6.63 & 48 & D & -- & -- & H & GWSI. \\
\hline 5,685 & 94 & 6.63 & 93 & D & -- & -- & H & GWSI. \\
\hline 5,740 & 60 & 6.63 & 60 & D & -- & -- & H & GWSI. \\
\hline 5,745 & 46 & 6.63 & 46 & $D$ & - & -- & H & GWSI. \\
\hline 5,780 & 78 & 6.63 & 78 & D & - & -- & $\mathrm{H}$ & GWSI. \\
\hline 5,735 & 72 & 6.00 & 72 & D & -- & -- & H & GWSI. \\
\hline 5,750 & 60 & 6.63 & 56 & D & -- & -- & H & GWSI. \\
\hline 5,775 & 51 & 6.63 & 51 & D & -- & -- & $\mathrm{H}$ & GWSI. \\
\hline 5,780 & 53 & 6.63 & 53 & D & -- & - & $\mathrm{H}$ & GWSI. \\
\hline 5,820 & 27 & 6.63 & 27 & D & -- & -- & $\mathrm{H}$ & GWSI. \\
\hline 5,750 & 55 & 6.00 & 55 & D & - & -- & H & GWSI. \\
\hline 5,780 & 45 & 6.63 & 44 & D & -- & -- & H & GWSI. \\
\hline 5,840 & 98 & 6.00 & 98 & D & -- & -- & H & GWSI. \\
\hline 5,750 & 46 & 6.63 & 46 & D & -- & -- & H & GWSI. \\
\hline
\end{tabular}




\begin{tabular}{|c|c|c|c|c|c|c|}
\hline $\begin{array}{l}\text { Number } \\
\text { on } \\
\text { plate } 2\end{array}$ & $\begin{array}{l}\text { Lati- } \\
\text { tude }\end{array}$ & $\begin{array}{l}\text { Longi- } \\
\text { tude }\end{array}$ & Local identifier & Type & Owner & $\begin{array}{l}\text { Principal } \\
\text { aquifer }\end{array}$ \\
\hline 366 & 360723 & 1064408 & 22N.02E.21.321 & $\mathrm{SP}$ & -- & -- \\
\hline 367 & 360742 & 1060941 & $22 \mathrm{~N} .07 \mathrm{E} .23 .213$ & GW & -- & 110AVKB \\
\hline 368 & 360748 & 1063656 & 22N.03E.22.111 & SP & -- & 231CHNL \\
\hline 369 & 360807 & 1061007 & 22N.07E.14.314 & GW & Grt Plains Land & 121TSUQ \\
\hline 370 & 360949 & 1062633 & Polvadera Grant & SP & -- & -- \\
\hline 371 & 360957 & 1062119 & Town of Abiquiu Grant Agua Caliente & SP & -- & -- \\
\hline 372 & 360958 & 1062116 & Town of Abiquiu Grant & SP & -- & -- \\
\hline 373 & 361001 & 1063632 & $22 \mathrm{~N} .03 \mathrm{E} .03 .322$ & GW & Garcia, Felix & 110AVIB \\
\hline 374 & 361006 & 1063706 & 22N.03E.04.411 & GW & -- & 231CHNL \\
\hline 375 & 361033 & 1061056 & $23 \mathrm{~N} .07 \mathrm{E} .34 .340$ & GW & Gallegos, Jose $\mathrm{K}$. & 110AVKB \\
\hline 376 & 361038 & 1061147 & 23N.07E.33.431 & GW & Herrera, Felix & 110AVKB \\
\hline 377 & 361039 & 1061110 & 23N.07E. 34.332 & GW & Martinez, Pedro L. & -- \\
\hline 378 & 361059 & 1063302 & $23 \mathrm{~N} .04 \mathrm{E} .31 .234$ & GW & Ydwca Youngsvll & 110AVYB \\
\hline 379 & 361110 & 1062347 & Juan Bautista Valdez Grant & GW & -- & 112SNTF \\
\hline 380 & 361115 & 1061337 & 23N.07E. 31.220 & GW & Valdez, Fred & $110 \mathrm{AVWB}$ \\
\hline 381 & 361116 & 1063324 & 23N.04E. 31.131 & GW & -- & 231CHNL \\
\hline 382 & 361117 & 1063343 & 23N.04E.31.111 & GW & -- & 110AVMB \\
\hline 383 & 361122 & 1063326 & 23N.04E.31.112 & GW & -- & $110 \mathrm{AVKB}$ \\
\hline 384 & 361137 & 1061326 & 23N.07E. 29.313 & GW & Montoya, Jose L. & - \\
\hline 385 & 361144 & 1061407 & 23N.07E. 30.322 & GW & Smith, Floyd & $110 \mathrm{AVYB}$ \\
\hline 386 & 361147 & 1061413 & Lobato Grant & $G W$ & -- & 110AVYB \\
\hline 387 & 361207 & 1061409 & 23N.07E. 30.120 & GW & Vlahos, Barbara & 110AVKB \\
\hline 388 & $3612 \quad 24$ & 1061838 & $23 \mathrm{~N} .06 \mathrm{E} .20 .444$ & GW & Mdwswa Abiquiu & -- \\
\hline 389 & 361224 & 1061850 & Town of Abiquiu Grant & GW & -- & $112 \mathrm{SNTF}$ \\
\hline 390 & 361231 & 1061853 & Town of Abiquiu Grant & GW & -- & 110AVIB \\
\hline 391 & 361235 & 1062126 & $23 \mathrm{~N} .05 \mathrm{E} .24 .300$ & GW & Garcia, Ben & - \\
\hline 392 & $36 \quad 1252$ & 1061425 & 23N.07E. 19.000 & GW & SW Land corp. & $110 \mathrm{AVNB}$ \\
\hline 393 & 361315 & 1063005 & Piedra Lumbre Grant & GW & -- & $110 \mathrm{AVYB}$ \\
\hline 394 & 361359 & 1062306 & $23 \mathrm{~N} .05 \mathrm{E} .15 .212$ & GW & -- & - \\
\hline
\end{tabular}




\begin{tabular}{|c|c|c|c|c|c|c|c|c|}
\hline $\begin{array}{l}\text { Altitude } \\
\text { of land- } \\
\text { surface } \\
\text { datum } \\
\text { (feet above } \\
\text { sea level) }\end{array}$ & $\begin{array}{c}\text { Total } \\
\text { depth } \\
\text { of } \\
\text { well } \\
\text { (feet) }\end{array}$ & $\begin{array}{l}\text { Casing } \\
\text { diameter } \\
\text { (inches) }\end{array}$ & $\begin{array}{l}\text { Depth } \\
\text { cased } \\
\text { (feet) }\end{array}$ & $\begin{array}{l}\text { Data } \\
\text { avail- } \\
\text { able }\end{array}$ & $\begin{array}{c}\text { Number } \\
\text { of } \\
\text { analyses }\end{array}$ & $\begin{array}{l}\text { Period } \\
\text { of } \\
\text { record }\end{array}$ & $\begin{array}{l}\text { Water } \\
\text { use }\end{array}$ & $\begin{array}{l}\text { Source of data and additional data sources } \\
\text { (site name if other than WATSTORE name) }\end{array}$ \\
\hline- & - & - & -- & A & 1 & 1953 & -- & Qh. \\
\hline-- & - & -- & -- & $A, B$ & 1 & 1975 & -- & QW, T1978 (X13). \\
\hline-- & - & -- & -- & $A, B$ & 1 & 1974 & -- & QN, T1978 (U2). \\
\hline 5,880 & 490 & 6.63 & 490 & $\mathrm{D}$ & - & - & H & GWSI. \\
\hline- & - & - & -- & $A, B$ & 1 & 1974 & -- & QN, T1978 (V3). \\
\hline- & - & - & - & A & 1 & 1974 & - & QW, T1978 (V2). \\
\hline- & -- & -- & - & $A, B$ & 2 & 1974 & -- & QN, T1978 (V1). \\
\hline 6,700 & 50 & 8.00 & 50 & $A$ & 1 & 1964 & H & QW, GWSI, T1978 (UA3). \\
\hline- & - & -- & - & $A, B$ & 1 & 1975 & -- & QN, T1978 (UA5). \\
\hline 5,840 & 63 & 6.63 & 63 & $D$ & -- & -- & 且 & GWSI. \\
\hline 5,810 & 50 & 6.63 & 50 & D & - & - & $\mathrm{H}$ & GHSI. \\
\hline 5,830 & 50 & 6.25 & 50 & D & - & - & H & GHSI. \\
\hline 6,980 & 150 & 6.63 & 150 & $A, B, D$ & 1 & 1964 & $P$ & QH, GWSI, T1978 (UB5). \\
\hline- & -- & -- & -- & $A, B$ & 1 & 1975 & -- & QN, T1978 (V5). \\
\hline 5,880 & 50 & 6.63 & 50 & D & - & - & H & GHSI. \\
\hline- & - & - & -- & $A, B$ & 1 & 1975 & - & QW, T1978 (UB4). \\
\hline- & -- & -- & -- & $A, B$ & 1 & 1975 & -- & QNi, T1978 (UB2). \\
\hline-- & -- & -- & -- & $A, B$ & 1 & 1975 & - & QW, T1978 (UB3). \\
\hline 5,850 & 43 & 6.63 & 43 & D & - & -- & H & GWSI. \\
\hline 5,875 & 56 & 6.63 & 56 & D & -- & -- & В & GWSI. \\
\hline- & - & - & -- & $A, B$ & 1 & 1975 & - & QN, T1978 (X2). \\
\hline 5,865 & 53 & 6.63 & 53 & $D$ & - & - & H & GWSI. \\
\hline 6,000 & -- & -- & -- & $A, B$ & 1 & 1964 & $P$ & QH, GWSI. \\
\hline- & -- & -- & -- & $A, B$ & 1 & 1975 & -- & QW, T1978 (W3). \\
\hline- & - & - & -- & $A, B$ & 1 & 1975 & - & QW, T1978 (W4). \\
\hline 6,490 & 63 & 6.00 & 63 & D & -- & -- & H & GWSI. \\
\hline 5,900 & 78 & 6.63 & 78 & D & -- & -- & H & GWSI. \\
\hline- & -- & -- & -- & $A, B$ & 1 & 1975 & -- & QW. \\
\hline-- & -- & -- & -- & $A, B$ & 1 & 1974 & -- & QN, T1978 (V4). \\
\hline
\end{tabular}

